\title{
2021
}

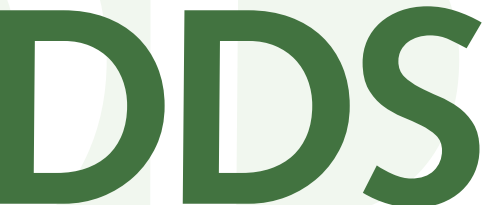

Die Deutsche Schule

Zeitschrift für Erziehungswissenschaft, Bildungspolitik und pädagogische Praxis

Herausgegeben von der Gewerkschaft Erziehung und Wissenschaft

\section{Führungskräfte gewinnen}

Schwerpunkt Colin Cramer, Jana Groß Ophoff, Marcus Pietsch \& Pierre Tulowitzki Schulleitung in Deutschland Repräsentative Befunde zur Attraktivität, zu Karrieremotiven und zu Arbeitsplatzwechselabsichten

Berichte zum Stephan Gerhard Huber \& Nadine Schneider

Schwerpunkt Durchgängige und systematische schulische Führungskräftegewinnung und -entwicklung

Christiane Montandon \& Marie Brégeon

Rekrutierung, Qualifizierung, Status und Karriereoptionen von Schulleiter*innen staatlicher Sekundarschulen in Frankreich

\section{Diskussion zum Katja Kansteiner, Pierre Tulowitzki,}

Schwerpunkt Michael Krüger \& Christoph Stamann

Führungskräfteentwicklung in der Lehramtsausbildung

Thesendiskussion zu curricularen Ergänzungen

des Lehramtsstudiums

Martin Rothland

Die "Lehrerpersönlichkeit" : das Geheimnis des Lehrberufs?

Weiterer Beitrag

Anje Ostermann, Anke Lindmeier, Hendrik Härtig, Lorenz Kampschulte, Mathias Ropohl \& Julia Schwanewedel Mathematikspezifische Medien nutzen: Was macht den Unterschied - Lehrkraft, Schulkultur oder Technik?

Diskussion Hans-Jürgen Kuhn \& Michael Voges

Blindflug beenden und stark aus der Krise kommen

Detlef Fickermann

Daten für Taten 


\section{Die Deutsche Schule Zeitschrift für Erziehungswissenschaft, Bildungspolitik und pädagogische Praxis}

Herausgeber: Gewerkschaft Erziehung und Wissenschaft im DGB in Zusammenarbeit mit der Max-Traeger-Stiftung

Redaktion: Dr. Götz Bieber (Ludwigsfelde), Prof. Dr. Kathrin Dedering (Erfurt), Benjamin Edelstein (Berlin), Detlef Fickermann (Kamen), Prof. Dr. Julia Gerick (Braunschweig), Prof. Dr. Marianne Krüger-Potratz (Münster), Dr. Veronika Manitius (Soest), Prof. Dr. Kathrin Racherbäumer (Siegen), Jun.-Prof. Dr. Verena Schreiber (Freiburg i. Br.)

Geschäftsführerin: Dr. Monika Palowski-Göpfert, Universität Bielefeld, Fakultät für Erziehungswissenschaft, AG 4, Konsequenz 41a, 33615 Bielefeld, E-Mail: dds@uni-bielefeld.de Vorsitzender der Redaktion: Detlef Fickermann (Kamen)

Beirat: Prof. Dr. Herbert Altrichter (Linz-Auhof), Dr. Christine Biermann (Bielefeld), Marianne Demmer (Wilnsdorf), Prof. Dr. Mats Ekholm (Karlstad), Prof. Dr. Friederike Heinzel (Kassel), Prof. Dr. Thomas Höhne (Hamburg), Prof. Dr. Klaus Klemm (Essen), Prof. Dr. Eckhard Klieme (Frankfurt a. M.), Prof. Dr. Katharina Maag Merki (Zürich), Prof. Dr. Heinrich Mintrop (Berkeley), Prof. Dr. Angelika Paseka (Hamburg), Prof. Dr. Nicolle Pfaff (Essen), Prof. Dr. Sabine Reh (Berlin), Prof. Dr. Hans-Günter Rolff (Dortmund), Prof. Andreas Schleicher (Paris), Jochen Schweitzer (Münster), Ulrich Steffens (Wiesbaden), Prof. Dr. KlausJürgen Tillmann (Berlin)

Beitragseinreichung und Double-blind Peer Review: Manuskripte (nur Originalbeiträge) werden als Word-Datei an die Geschäftsführung (dds@uni-bielefeld.de) erbeten. Bitte beachten Sie die Hinweise zur Manuskriptgestaltung (www.dds-home.de). Seit dem 103. Jahrgang (2011) durchlaufen alle Fachartikel in der DDS (Texte zum Themenschwerpunkt und für die Rubrik „Weitere Beiträge“) ein externes Review-Verfahren. Nach einer redaktionellen Prüfung der eingereichten Aufsätze im Hinblick auf ihre grundsätzliche Eignung für die DDS schließt sich eine Begutachtung im Doppelblindverfahren durch ehrenamtlich tätige Gutachter*innen an.

Die Deutsche Schule erscheint vierteljährlich. Zusätzlich zu den vier Heften pro Jahrgang können Beihefte erscheinen. Unter www.waxmann.com und www.dds-home.de finden Sie weitere Informationen. Die DDS ist indiziert in ESCI, FIS Bildung und Proquest und für weitere Indizierungen vorgeschlagen.

Preise und Bezugsbedingungen: Jahresabonnement 59,00 €, für GEW-Mitglieder/Studierende 43,00 €. Die Preise verstehen sich zzgl. Versandkosten. Abbestellungen spätestens 6 Wochen vor Ablauf des Jahresabonnements. Online erscheint die Zeitschrift open access unter der Lizenz CC BY-NC-ND 4.0.

ISSN 0012-0731

E-ISSN 2699-5379

(C) Waxmann Verlag GmbH, 2021

Steinfurter Straße 555, 48159 Münster, Telefon: 02 51/2 6504 0, Fax: 02 51/2 6504 26, Internet: www.waxmann.com, E-Mail: info@waxmann.com

Anzeigenverwaltung: Waxmann Verlag GmbH, Paula Brauer: brauer@waxmann.com Druck: mediaprint solutions $\mathrm{GmbH}$, Paderborn

Satz: Roger Stoddart, Münster

Die Zeitschrift und alle in ihr enthaltenen Beiträge und Abbildungen sind urheberrechtlich geschützt. Jede Verwendung außerhalb der engen Grenzen des Urheberrechtsgesetzes ist ohne Zustimmung des Verlags unzulässig und strafbar. Unter dieses Verbot fallen insbesondere die gewerbliche Vervielfältigung per Kopie, die Aufnahme in elektronische Datenbanken und die Vervielfältigung auf CD-Rom und allen anderen elektronischen Datenträgern. 
DDS - Die Deutsche Schule Zeitschrift für Erziehungswissenschaft, Bildungspolitik und pädagogische Praxis https://doi.org/10.31244/dds.2021.02

113. Jahrgang 2021 / Heft 2

\section{INHALT}

\section{EDITORIAL}

Götz Bieber \& Marianne Krüger-Potratz

Editorial zum Schwerpunktthema: Führungskräfte gewinnen 127

\section{FÜHRUNGSKRÄFTE GEWINNEN}

Colin Cramer, Jana Groß Ophoff, Marcus Pietsch \& Pierre Tulowitzki

Schulleitung in Deutschland

Repräsentative Befunde zur Attraktivität, zu Karrieremotiven

und zu Arbeitsplatzwechselabsichten... 132

\section{BERICHTE ZUM SCHWERPUNKTTHEMA}

Stephan Gerhard Huber \& Nadine Schneider

Durchgängige und systematische schulische Führungskräftegewinnung

und -entwicklung

Christiane Montandon \& Marie Brégeon

Rekrutierung, Qualifizierung, Status und Karriereoptionen von

Schulleiter*innen staatlicher Sekundarschulen in Frankreich 161

\section{DISKUSSION ZUM SCHWERPUNKTTHEMA}

Katja Kansteiner, Pierre Tulowitzki, Michael Krüger \& Christoph Stamann

Führungskräfteentwicklung in der Lehramtsausbildung

Thesendiskussion zu curricularen Ergänzungen des Lehramtsstudiums 174

Martin Rothland

Die „Lehrerpersönlichkeit“: das Geheimnis des Lehrberufs? 188 


\section{WEITERER BEITRAG}

Anje Ostermann, Anke Lindmeier, Hendrik Härtig, Lorenz Kampschulte,

Mathias Ropohl \& Julia Schwanewedel

Mathematikspezifische Medien nutzen: Was macht den

Unterschied - Lehrkraft, Schulkultur oder Technik?

\section{DISKUSSION}

Hans-Jürgen Kuhn \& Michael Voges

Blindflug beenden und stark aus der Krise kommen

Bildungschancen für Benachteiligte jetzt sichern!

\section{Detlef Fickermann}

\section{Daten für Taten}

Verbesserung der Datengrundlagen für zielgerichteteres

politisches Handeln zur Eindämmung und Bewältigung der

Folgen der Corona-Pandemie.

\begin{tabular}{ll}
\hline 3021 & Vorschau \\
Themenschwerpunkt: Kooperation von Eltern und Schule?! \\
In der aktuellen Situation des Distanzunterrichts wird die Bedeutung der \\
Unterstützung der Kinder durch ihre Eltern für das schulische Lernen \\
besonders evident. Aber auch jenseits pandemischer Verhältnisse wurde \\
vielfach nachgewiesen, dass sich die Zusammenarbeit von Schule und \\
Eltern positiv auf den Bildungsverlauf der Kinder auswirkt. Dabei ist das \\
Zusammenspiel dieser beiden Akteursgruppen in Schule eingebettet in \\
ein komplexes Geflecht von gesellschaftlichen Diskursen, schulkultureller \\
Traditionen bis hin zu individuellen Erwartungen, Vorerfahrungen aber \\
auch rechtlichen Vorgaben. Das nächste Heft gibt einen Überblick über \\
den aktuellen Forschungsstand zur Kooperation von Eltern und Schule. \\
Ausgewählte Befunde aktueller Forschungsprojekte bieten vertiefende \\
Einblicke zu wichtigen Aspekten wie etwa Vertrauen von Eltern in die Bil- \\
dungsinstitution Schule oder Wirkungen elterlicher Unterstützung beim \\
Lesekompetenzerwerb. Darüber hinaus beleuchten Berichte das Thema \\
Eltern und Schule aus unterschiedlichen Akteursperspektiven. \\
Heft 3 erscheint im August 2021. \\
Waxmann • Steinfurter Str. $555 \bullet 48159$ Münster • www.waxmann.com
\end{tabular}


DDS - Die Deutsche Schule Zeitschrift für Erziehungswissenschaft, Bildungspolitik und pädagogische Praxis https://doi.org/10.31244/dds.2021.02

113. Jahrgang 2021 / Heft 2

\title{
CONTENTS
}

\author{
EDITORIAL
}

Götz Bieber \& Marianne Krüger-Potratz

Editorial to the Focus Topic: Recruiting Educational Leaders 127

\section{RECRUITING EDUCATIONAL LEADERS}

Colin Cramer, Jana Groß Ophoff, Marcus Pietsch \& Pierre Tulowitzki

School Principals in Germany

Representative Findings on Attractiveness, Career Motives and

Turnover Intentions 132

\section{REPORTS ON THE FOCUS TOPIC}

Stephan Gerhard Huber \& Nadine Schneider

Consistent and Systematic Recruitment and Development

of School Leaders

Christiane Montandon \& Marie Brégeon

Recruitment, Qualification, Status and Career Options of Headteachers for the Secondary School Forms in France 161

\section{DISCUSSION ON THE FOCUS TOPIC}

Katja Kansteiner, Pierre Tulowitzki, Michael Krüger \& Christoph Stamann

Leadership Development in Teacher Education

Discussion of Theses Regarding a Curricular Expansion

of Teacher Education

Martin Rothland

The “Teacher Personality": Secret of the Teaching Profession? 188 


\section{FURTHER CONTRIBUTION}

Anje Ostermann, Anke Lindmeier, Hendrik Härtig, Lorenz Kampschulte, Mathias Ropohl \& Julia Schwanewedel

Using Mathematics-specific Media: What Makes the Difference Teachers, School-Culture, or Equipment?

\section{DISCUSSION}

Hans-Jürgen Kuhn \& Michael Voges

Quitting the Blind Flight and Coming Out of the Crisis Strong

Secure Educational Opportunities for the Disadvantaged now!

\section{Detlef Fickermann}

\section{Data to Act Upon}

Improving the Data Basis for Targeted Political Action to Contain and Overcome the Consequences of the Corona Pandemic

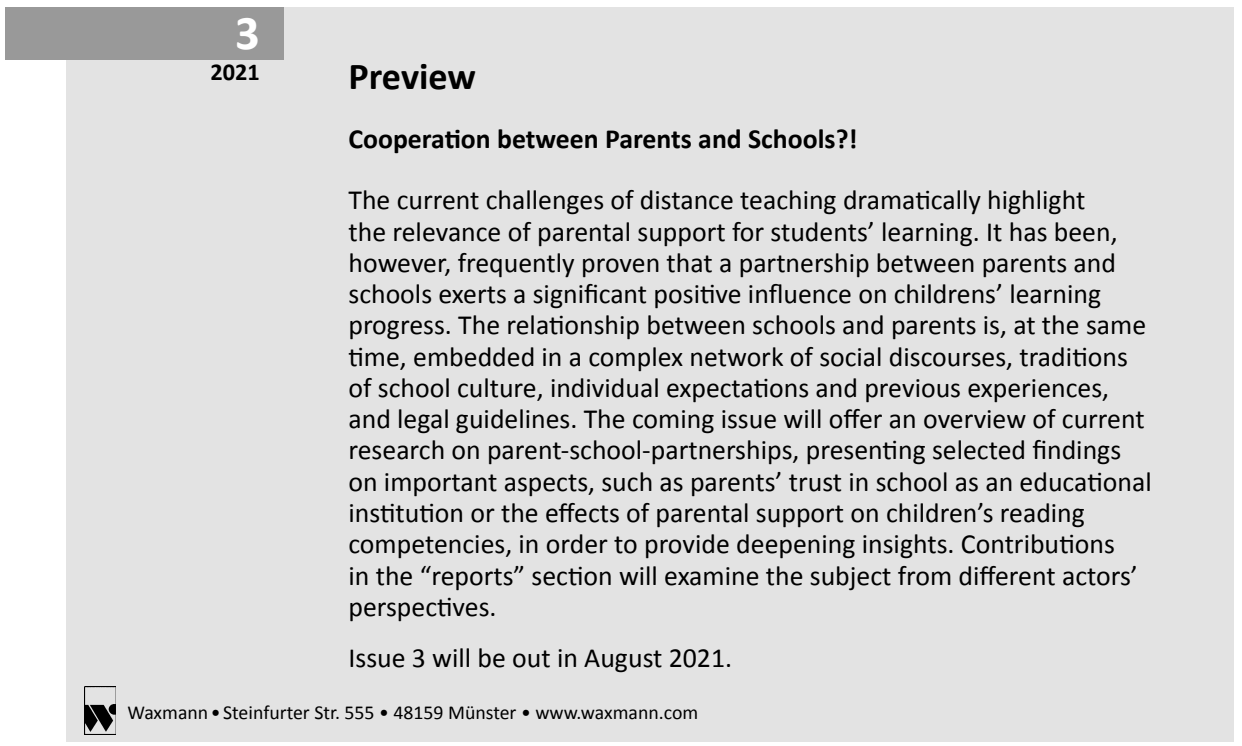




\section{EDITORIAL}

\section{Editorial zum Schwerpunktthema: Führungskräfte gewinnen}

\section{Editorial to the Focus Topic: Recruiting Educational Leaders}

An schulische Führungskräfte werden hohe Anforderungen gestellt, die sich aus ihren, im Zuge der letzten Jahrzehnte deutlich erweiterten, Aufgaben hinsichtlich der inhaltlichen und organisatorischen Steuerung der Schule nach innen wie nach außen ergeben. Zentrale Entwicklungsaufgaben, wie die Umsetzung der notwendigen Veränderungen für eine inklusive und digital gestützte Schule angesichts der Herausforderungen in einer zunehmend digital geprägten Welt, bestimmen die strategische Arbeit der schulischen Führungskräfte. Dabei liegt der Fokus auf einer stärker am Individuum ausgerichteten Unterrichtsgestaltung, auch unter Nutzung der Potenziale digitaler Medien und Werkzeuge. Um allein dieses Ziel zu erreichen, müssen Prozesse der Personalentwicklung, u.a. durch Angebote in der Qualifizierung wie durch Fortbildung des pädagogischen Personals der Schule, initiiert bzw. ausgebaut werden. Gleichzeitig müssen die Organisationsprozesse in der Schule durch Digitalisierung effizienter und die Kooperation im Kollegium nachhaltig gestaltet werden.

Aktuell dominiert die Frage, wie es gelingen kann, schulische Bildung in der Pandemiesituation weiterhin so anbieten zu können, dass die Schülerinnen die Jahresziele erreichen. Dies erweckt den Eindruck, als würden derzeit alle anderen Aufgaben von Führungskräften dahinter zurückstehen bzw. zurückgestellt werden. Doch genau das Gegenteil ist der Fall. Neben den weiterhin bestehenden Aufgaben müssen die Schulleiter*innen sich diesen und anderen Herausforderungen unter erschwerten Bedingungen in allen Bereichen der pandemiegeprägten Schulrealität und Schulentwicklung stellen: Unterricht und Unterrichtsentwicklung sind in Modellen $\mathrm{zu}$ organisieren und zu gestalten, in denen ganz oder zum Teil auf die Präsenz im Klassenraum verzichtet werden muss; die Organisation schulischer Prozesse sowie deren Entwicklung ist ohne oder nur mit geringen Anteilen an direkter Kommunikation im und mit dem Kollegium und den Gremien sicherzustellen, um den Fortgang der Arbeitsprozesse zu gewährleisten, aber auch, um die Motivation aller aufrecht zu erhalten. Bereitzustellen sind weiterhin Unterstützungs- und Qualifizierungsmaßnahmen, nun aber ohne Präsenz von außen; Kooperation als zentrales 
Element der Gestaltung von Arbeits- und Entwicklungsprozessen ist in überwiegend und zeitweise in ausschließlich digitaler Form aufrechtzuerhalten bzw. weiterzuentwickeln. Technologieentwicklung ist aktuell tägliche Realität in der Umsetzung. All das muss unter Beachtung und Abwägung des Gesundheitsschutzes aller an Schule Beteiligten gestaltet werden. Die fünf oben genannten Aspekte von Schulentwicklung (vgl. Eickelmann \& Gerick, 2018; Labusch, Eickelmann \& Conze, 2020) werden auch künftig, nach der Pandemie, für die Transformation des Bildungssystems in einer sich permanent verändernden Gesellschaft bedeutsam sein.

Daraus ergeben sich zwei entscheidende Herausforderungen, um sicherzustellen, dass es nicht an gut qualifizierten Führungskräften fehlt, und dies nicht nur auf nationaler, sondern auch auf europäischer Ebene. Denn - so die Europäische Kommission:

„Ohne Lehr- und Führungskräfte kann die schulische Bildung nicht existieren. Sie sind jeden Tag für die Entwicklung der Lernenden verantwortlich, in deren Interesse sie ihren Beruf ausüben. Die europäischen Schulbildungssysteme sind nicht nur darauf angewiesen, kontinuierlich genügend Stellen mit Lehr- und Führungskräften zu besetzen, sondern sie wollen diese auch halten und fördern. Gleichwohl wird über einen erheblichen Lehrermangel berichtet. Zudem wird die Laufbahnentwicklung in der schulischen Bildung oft noch immer als flach oder eindimensional mit nur geringen oder gar keinen Aufstiegsmöglichkeiten gesehen. Dies stellt für die Schulen und die Bildungssysteme eine enorme Herausforderung dar, denn sie brauchen nicht nur nachhaltige und hochkompetente Berufsprofile, sondern auch leidenschaftliche und zukunftsorientierte Menschen, die diesen Beruf ausüben“ (GD EAC, 2020, S. 2).

Um dies zu erreichen, muss die Qualifizierung von Schulleitungen weiter ausgebaut und auf die o.g. Anforderungen ausgerichtet werden. Zugleich gilt es, die Gewinnung geeigneter Personen für Führungsaufgaben zu verstärken und Qualifizierungsangebote zu entwickeln, die nicht nur auf den Stellen- und Statuswechsel, sondern auch darauf zugeschnitten sind, dass Personen frühzeitig in ihrer Karriere, ggf. auch zeitlich begrenzt, Führungsaufgaben in kleinerem Rahmen übernehmen. Ein erster Schritt ist insofern getan, als die Qualifizierung und Fortbildung für Schulleitungen sowohl vor dem Amt als auch tätigkeitsbegleitend in den letzten Jahren immer stärker ins Bewusstsein aller Beteiligten gelangt ist (vgl. Klein, Richter \& van Ackeren, 2020). Für weitere Schritte sind die Ergebnisse der Studien von Interesse, die auch auf die Frage eingehen, wie das Personal für diese anspruchsvollen Führungsaufgaben gewonnen werden kann. Die aktuellen Vorschläge, die auch in diesem Heft vertreten und diskutiert werden, reichen von der Entwicklung von ersten Maßnahmen in der Lehrkräftebildung bis hin zu Modellen einer teilweise gemeinsamen Qualifizierung von Schulleitungen und Schulaufsicht.

Die hier angesprochenen Themen werden im vorliegenden Heft unter verschiedenen Gesichtspunkten aufgegriffen. Ausgehend von ihrem Projekt „Schulleitungen in Deutschland“ - einer Online-Befragung von 405 Lehrkräften und Schulleitungen - 
gehen Colin Cramer, Jana Groß Ophoff, Marcus Pietsch und Pierre Tulowitzki der Frage nach, warum Lehrkräfte zögern, sich für eine Schulleitung zu bewerben, obwohl sie an der Aufgabe selbst interessiert sind, und wie Schulleiter*innen ihre Karriere weiterentwickeln möchten. Deutlich wird, dass es nicht an Interesse fehlt weder bei Lehrkräften noch bei den Schulleitungen. Aber in beiden Fällen wird auf die Arbeitsrealität verwiesen: seitens der Lehrkräfte, um zu erklären, warum sie sich - trotz Interesse - letztlich doch (noch) nicht für einen Karrierewechsel entschieden haben, und seitens der Schulleitungen, von denen immerhin 20 Prozent angaben, einen Arbeitsplatzwechsel in Erwägung zu ziehen. Sie dürften allerdings mehrheitlich zugleich zu den 95 Prozent der Schulleitungen gehören, die erklärten, dass sie Freude an ihrer Arbeit haben bzw. zu den 88 Prozent, die ihre Arbeit als inspirierend bezeichneten.

In der Rubrik „Berichte“ befassen sich Stephan Gerhard Huber und Nadine Schneider mit den vielfältigen Aufgaben von Leadership auf den verschiedenen Ebenen des Bildungssystems und den dafür notwendigen Kompetenzen und Qualifizierungen. Gestützt auf eine langjährige Beschäftigung mit dem Thema Personalmanagement in Forschung und wissenschaftlicher Beratung einerseits und speziell bezogen auf ein Kooperationsprojekt der Pädagogischen Hochschule Zug mit zwei großen Stiftungen andererseits, setzen sie sich mit der Frage der frühzeitigen Gewinnung von Führungspersonal und der (teilweisen) Verzahnung von Qualifizierungsmaßnahmen auseinander. Sie plädieren für eine „durchgängige und systematische schulische Führungskräftegewinnung und -entwicklung“ - so auch der Titel ihres Beitrags. Unter „durchgängig“ verstehen sie zum einen, dass Fragen der Führung schon in den Phasen der Lehrkräftebildung thematisiert werden sollten, und zum anderen, dass die Qualifizierung von Schulleitung und Schulaufsicht in bestimmten Teilen miteinander verbunden werden sollte. Ziel müsse ein besseres Zusammenspiel von bislang hierarchisch getrennten Berufen angesichts gemeinsamer Ziele bei geteilter Verantwortung sein.

Mit dem von Christiane Montandon und Marie Brégeon verfassten Beitrag richtet sich der Blick nach Frankreich und auf die dortige zentral gesteuerte Führungskräftegewinnung und -qualifizierung. Unter dem Titel „Rekrutierung, Qualifizierung, Status und Karriereoptionen von Schulleiter*innen staatlicher Sekundarschulen in Frankreich" wird ein hierarchisch und zentral organisiertes und gesteuertes System der Rekrutierung, Qualifizierung und Beschäftigung von Schulleitungen vorgestellt. Die Autorinnen gehen auf die in den letzten Jahren erfolgten Veränderungen der Qualifizierung der Schulleitungen in Theorie und Praxis ein. Dazu gehört auch die mit dem Stichwort „personalisierte Ausbildung“ verbundene Aufforderung, dass die zukünftigen Schulleiter*innen sich mit Unterstützung des ihnen an ihrer „Ausbildungsschule“ zugewiesenen Mentors von Beginn an Gedanken über den weiteren Verlauf ihrer Karriere machen sollen, z.B. darüber, an welcher Schule sie sich zukünftig um einen Schulleitungsposten bewerben wollen, da - je nach Schulform, 
Lage der Schule, Besonderheiten des Angebots usw. - nicht nur die Aufgaben und Anforderungen, sondern auch die Höhe der Zulagen variieren. Ergänzt wird diese Darstellung durch Aussagen von zwei Schulleitern, die im Rahmen einer Interviewstudie auf ihre Karriere zurückblicken.

In der Rubrik „Zur Diskussion“ greifen Katja Kansteiner, Pierre Tulowitzki, Michael Krüger und Christoph Stamann das im Bericht von Huber und Schneider angesprochene Thema der „Führungskräfteentwicklung in der Lehramtsausbildung“ in Form einer „Thesendiskussion zu curricularen Ergänzungen des Lehramtsstudiums“ auf. Aus ihrer Sicht ist die Aufnahme der Themen Personalführung und Management in die Kompetenzstandards für eine auf die aktuellen Herausforderungen ausgerichtete wissenschaftliche Lehrkräftebildung zwingend; zugleich sehen sie darin eine Chance für eine frühzeitige Führungskräftegewinnung und -entwicklung. In ihrem Beitrag skizzieren sie anhand schon existierender MA-Weiterbildungsstudiengänge zu Personalführung und Management, wie sie sich entsprechende Angebote in der ersten Phase der Lehramtsausbildung vorstellen.

Unter dem Titel „Die ,Lehrerpersönlichkeit': das Geheimnis des Lehrberufs?“ setzt sich Martin Rothland mit einer Art „Gegenkonzept“ zur Diskussion über Konzepte der Qualifizierung und Qualifizierbarkeit von Führungskräften auseinander: mit dem „Mythos“, man müsse zur Lehrkraft berufen sein. Dass diese Idee immer noch populär ist, belegt er durch zahlreiche Beispiele aus der neueren Literatur, in der die ”Lehrerpersönlichkeit“ entweder - in der Tradition der geisteswissenschaftlichen Pädagogik - als „Wesensmerkmal“ definiert wird oder als „Gegenstand und Ziel der Lehrerbildung und Professionalisierung“ unter „Persönlichkeitsbildung“ und somit als auch „erlernbar“ gefasst wird. Aber selbst in den letztgenannten Texten werde daran festgehalten, dass die „Lehrerpersönlichkeit“ empirisch nicht erfassbar und damit nicht erklärbar und letztlich auch nicht lernbar sei. Folge man dieser Position, so sei „die Forderung, Professionalisierung für und Professionalität im Lehrberuf an die Ausbildung der „Lehrerpersönlichkeit“ zu knüpfen, [...] nicht einzulösen“.

In einem fachlich ausgerichteten weiteren Beitrag geht es um die Frage, was den Unterschied bei der Nutzung „Mathematikspezifischer Medien“ mache: „Lehrkraft, Schulkultur oder Technik?" Ihr sind Anje Ostermann, Anke Lindmeier, Hendrik Härtig, Lorenz Kampschulte, Mathias Ropohl und Julia Schwanewedel im Rahmen eines Projekts zum "Medieneinsatz im mathematisch-naturwissenschaftlichen Unterricht“ nachgegangen. Auf der Basis der Ergebnisse ihrer - allerdings nicht repräsentativen - Lehrkräftebefragung diskutieren sie u.a. die Implikationen zur Förderung des Medieneinsatzes im Mathematikunterricht.

Aus aktuellem Anlass sind zwei weitere Diskussionsbeiträge in dieses Heft aufgenommen worden. Angesichts der Tatsache, dass nunmehr seit einem Jahr Schule pandemiebedingt in Form von Fern-, Hybrid- und Wechselunterricht gestaltet wird, wer- 
den sich Bildungsungerechtigkeiten wohl weiter verschärfen und verfestigen. Um dem entgegen wirken zu können, fordern Hans-Jürgen Kuhn und Michael Voges in ihrem Diskussionsbeitrag zum einen Forschung zur Gewinnung gesicherter Erkenntnisse über die kognitiven, sozialen und psychischen Auswirkungen der pandemiebedingten Schulschließungen und zum anderen diagnosebasierte, wissenschaftlich fundierte Förderkonzepte. Ziel sollte ihres Erachtens die Aufstellung eines umfassenden Förderprogramms sein, um die Folgen der Pandemie auffangen und überwinden zu können.

Hierzu bedarf es weiterer und anderer Daten zum Infektionsgeschehen und vorhandene Daten aus verschiedenen anderen Bereichen müssen ergänzend für vertiefte Analysen genutzt werden. Detlef Fickermann benennt in seinem Diskussionsbeitrag Datenerfordernisse für ein geplantes Bund-Länder-Programm zur Schließung von coronabedingten Lern- bzw. Kompetenzlücken der Schüler*innen und für eine Evaluation eines solchen Programms. Ferner skizziert er Ansätze zur Identifikation von kleinräumigen Infektionsclustern und zu berufsgruppen- und branchenspezifischen Auswertungen unter Nutzung von zusätzlichen Datenquellen. Seinen Vorschlägen gemein ist die zusätzliche Nutzung vorhandener Datensätze und die Inanspruchnahme eines vertrauenswürdigen Dritten, um datenschutzkonform (Individual-)Daten aus unterschiedlichen Quellen miteinander verknüpfen und für vertiefte Auswertungen als Grundlage für evidenzbasierte politische Entscheidungen nutzen zu können.

Götz Bieber und Marianne Krüger-Potratz

\section{Literatur und Internetquellen}

Eickelmann, B., \& Gerick, J. (2018). Herausforderungen und Zielsetzungen im Kontext der Digitalisierung von Schule und Unterricht. Teil 2: Fünf Dimensionen der Schulentwicklung zur erfolgreichen Integration digitaler Medien. SchulVerwaltung NRW, 29 (4), 111-115.

GD EAC (Generaldirektion der Europäischen Kommission für Bildung, Jugend, Sport und Kultur). (2020). Unterstützung von Lehr- und Führungskräften an Schulen - Ein Strategieleitfaden. Zusammenfassung. Luxemburg: Amt für Veröffentlichungen der Europäischen Kommission. https://doi.org/10.2766/45392

Klein, E. D., Richter, D., \& Ackeren, I. van (Hrsg.). (2020). Schulleitungsfortbildung - Stand und Desiderate. DDS - Die Deutsche Schule, 112 (3). Münster: Waxmann. https://doi. org/10.31244/dds.2020.03

Labusch, A., Eickelmann, B., \& Conze, D. (2020). ICILS 2018 \#Transfer. Gestaltung digitaler Schulentwicklung in Deutschland. Münster: Waxmann.

https://doi.org/10.31244/dds.2021.02.01 


\title{
FÜHRUNGSKRÄFTE GEWINNEN
}

DDS - Die Deutsche Schule

113. Jahrgang 2021, Heft 2, S. 132-148

https://doi.org/10.31244/dds.2021.02.02

CC BY-NC-ND 4.0 Waxmann 2021

Colin Cramer, Jana Groß Ophoff, Marcus Pietsch \& Pierre Tulowitzki

Schulleitung in Deutschland

\section{Repräsentative Befunde zur Attraktivität, zu Karrieremotiven und zu Arbeitsplatzwechselabsichten}

\section{Zusammenfassung}

Dieser Beitrag stellt repräsentative Befunde zu Karrieren von Schulleitung in Deutschland vor. Ein knappes Drittel der Lehrpersonen hat bereits über eine Schulleitungsposition nachgedacht, diesen Weg aber vorwiegend aus Gründen der Zufriedenheit mit der pädagogischen Arbeit und der befürchteten Beanspruchung nicht verfolgt. Schulleitungen ergreifen das Amt zuvorderst, um mehr Gestaltungsmöglichkeiten, Verantwortung und Autonomie zu haben. Trotz einer hohen Zufriedenheit mit der Arbeit insgesamt zieht derzeit ein Fünftel der Schulleitungen in Deutschland einen Arbeitsplatzwechsel in Erwägung. Die Befunde werden vor dem Hintergrund des virulenten Mangels an Schulleitungen diskutiert.

Schlüsselwörter: Schulleitung, Attraktivität, Karrieremotive, Arbeitsplatzwechselabsicht

\section{School Principals in Germany}

Representative Findings on Attractiveness, Career Motives and Turnover Intentions

\begin{abstract}
This article reports representative findings regarding the careers of school principals in Germany. Almost a third of teachers already thought about applying for a position as a school principal but decided otherwise, not only because they fear stress and strain, but also because they are content with their current pedagogical work. School principals choose their career first and foremost to widen their creative scope and to gain more responsibility and autonomy. Despite a high level of overall job satisfaction, one fifth of school principals in Germany has a turnover intention. The findings are discussed against the backdrop of the virulent shortage of school principals.
\end{abstract}

Keywords: leadership, attractiveness, career motives, turnover intentions 


\section{$1 \quad$ Einleitung}

Die Relevanz von Schulleitung für die Schulentwicklung und indirekt auch für Leistungen von Schüler*innen wird international (Day \& Sammons, 2013; Leithwood, Harris \& Hopkins, 2020; Leithwood \& Louis, 2012) wie national (Bonsen, 2016; Pietsch \& Tulowitzki, 2017) konstatiert. Angesichts der hohen Relevanz von Schulleitung ist erstaunlich, dass in Deutschland zwar vereinzelt Studien zum Schulleitungshandeln vorliegen (im Überblick z. B. Schwanenberg, Klein \& Walpuski, 2018), doch was das Amt der Schulleitung für Lehrpersonen attraktiv macht, warum Schulleitungen diese Position ergriffen haben und welche Gründe gegen einen Verbleib im Amt sprechen, ist bislang im deutschsprachigen Raum wenig erforscht, obgleich es recht häufig zu Schulleitungswechseln kommt (Pietsch, Tulowitzki \& Hartig, 2019). Dabei galten 2018 z. B. in Baden-Württemberg 251 Schulleitungspositionen als unbesetzt (Kramer \& Bauer, 2019); die dpa ging im Dezember 2019 von deutschlandweit mehr als 1.000 unbesetzten Stellen aus (Sadigh \& dpa, 2019). Vor dem Hintergrund dieses akuten Mangels an Schulleitungen wirft dieser Beitrag im Projekt Leadership in German Schools (LineS2020) die Frage auf: Was macht das Amt der Schulleitung (un)attraktiv und welche Karrieremotive und Arbeitsplatzwechselabsichten haben Schulleitungen?

\section{Forschungsstand}

In Deutschland - wie auch in anderen westeuropäischen Ländern oder den USA - liegt ein Mangel an Bewerbungen für das Amt der Schulleitung vor (Tulowitzki, 2019a). Grundsätzlich nehmen sich Schulleitende in Deutschland als erfolgreich wahr, vor allem im Bereich Administration, weniger in Personalführung oder Organisationsentwicklung (Schwanenberg et al., 2018). Trotz der zunehmend wichtigeren Aufgabe der Schulentwicklung wenden Schulleitende mindestens 30 Prozent ihrer Arbeitszeit für Administration und nur 16 Prozent für Aufgaben rund um Curriculum und Unterricht auf (OECD, 2018, S. 38). Entsprechend gilt in Deutschland das Aufgabenprofil als stark durch Verwaltung geprägt (Huber, Gördel, Kilic \& Tulowitzki, 2016, S. 176; Meyer, Richter, Marx \& Hartung-Beck, 2019). Ebenso gelten Arbeitsbelastung und Burnout-Risiko als hoch (Pont, Nusche \& Moorman, 2008, S. 177). Mehrere Studien verdeutlichen, dass Schulleitende in Deutschland besonderen Belastungen aufgrund struktureller Rahmenbedingungen ausgesetzt sind (z. B. Warwas, 2012; Brauckmann \& Herrmann, 2013; Dadaczynski \& Paulus, 2016). Studien aus den USA und Kanada zeigen wiederum negative Wirkungen der Arbeitsbelastung auf Motivation und Effektivität im Beruf (Federici \& Skaalvik, 2012; Wang, Pollock \& Hauseman, 2018; für Deutschland vgl. Huber, Wolfgramm \& Kilic, 2013). 
Die Motive von Lehrpersonen, sich (potenziell) für oder gegen das Schulleitungsamt $z u$ entscheiden, wurden in Deutschland bislang kaum untersucht. Müller, Hancock, Wang und Stricker (2017) widmeten sich der Thematik in einer internationalen, nicht repräsentativen vergleichenden Befragung (Deutschland - USA). Sie unterschieden drei Faktoren: Schulkultur (z.B. Wunsch nach mehr Beteiligung an Schüler*innenaktivitäten), Supervision/Unterstützung von Mitarbeiter*innen (z.B. Wunsch nach mehr Zeit für Entwicklungsaktivitäten) sowie Vergütung und Sozialleistungen (z.B. Wunsch nach höherer Bezahlung). Vom US-amerikanischen Sample unterschied sich die deutsche Stichprobe im Bedürfnis nach höherem Gehalt und besserer Vorqualifikation.

$\mathrm{Zu}$ zentralen Karrieremotiven von Schulleiter ${ }^{\star}$ innen zählen nach Müller et al. (2017) die (vermeintliche) Hoffnung, Schule gestalten, Einfluss auf die Lernchancen von Schüler ${ }^{\star}$ innen nehmen und Lehrpersonen unterstützen zu können - verbunden mit der Hoffnung auf mehr Autonomie und eine Besserstellung der eigenen Position (Hancock, Hary \& Müller, 2012; Hancock, Müller \& Wang, 2016; Hancock, Müller, Wang \& Hachen, 2019). Die US-amerikanischen Befunde lassen sich verkürzt mit dem Slogan „Deciding to lead: Loving the mission and doing it my way“ (Torres, 2018, S. 8) resümieren. Befunde für Deutschland verweisen ebenfalls auf (persönliche) Verbesserung bzw. Entwicklung als zentrales Motiv für das Ergreifen des Amtes (Brauckmann, 2014). So konnte Wissinger (1996) für bayerische Schulleitungen zeigen, dass diese mit ihrer Karriereentscheidung retrospektiv vor allem mehr Verantwortung tragen wollen, die Vielfalt der Leitungsaufgaben attraktiv erscheint und Freude an verwaltungsbezogenen Aufgaben besteht; höheres Einkommen, höhere Reputation und verstärkte Zusammenarbeit mit Erwachsenen werden hingegen als weniger bedeutsam erachtet.

Die Mehrbelastung durch erweiterte Aufgaben in der Schulleitung (z.B. Brauckmann, 2014) wirft zudem die Frage nach Arbeitsplatzwechselabsichten von Schulleiter ${ }^{\star}$ innen bzw. nach Gründen für den Verbleib im Beruf auf. Tran (2017) zeigt anhand US-amerikanischer Daten, dass eine höhere Zufriedenheit mit dem Gehalt die Wahrscheinlichkeit einer Wechselabsicht verringert, während etwa die Schüler ${ }^{\star}$ innenklientel, die Schulgröße oder die Erfahrung der Befragten keinen signifikanten Einfluss haben. Eine Studie von Tekleselassie und Villarreal (2010) unterstreicht die Bezahlung als Einflussfaktor; zusätzlich geraten jedoch Berufszufriedenheit, Autonomieerleben und individuelle Merkmale in den Blick. Boyce und Bowers (2016) unterscheiden zwei Schulleitungstypen, die ihren Arbeitsplatz verlassen: solche, die zufrieden sind und einen Wechsel als Chance sehen, Gehalt, Handlungsspielräume und Herausforderungen zu vergrößern, und die es deshalb aus dem aktuellen Amt zieht („pull“) - und jene Unzufriedenen, die politische Rahmenbedingungen für problematisch erachten, zwischenmenschliche Konflikte und schlechte Arbeitsbeziehungen sehen oder persönliche Gründe anführen, warum sie sich aus dem Amt gedrängt („push“) fühlen. 
Schließlich existieren angesichts der im internationalen Vergleich eher kurzen Qualifizierung von Schulleitungen in Deutschland z.T. beträchtliche Unterschiede zwischen den Bundesländern bezüglich des Verpflichtungsgrades, Umfanges sowie der Modalitäten der Maßnahmen (Tulowitzki, Hinzen \& Roller, 2019). Ebenso stellt sich die berufliche Entwicklung von Schulleitungen, etwa mit Blick auf die einschlägigen Fortbildungsangebote, heterogen dar (Klein \& Tulowitzki, 2020).

\section{Methode}

Die repräsentativen Daten zu Lehrpersonen und Schulleitungen in Deutschland wurden durch den Felddienstleister forsa $\mathrm{GmbH}$ erhoben. Gefördert wurde die Durchführung der Studie durch die Qualitätsoffensive Lehrerbildung von Bund und Ländern aus Mitteln des Bundesministeriums für Bildung und Forschung (FKZ: 01JA1611).

\subsection{Grundgesamtheit, Stichprobe und Durchführung der Studie}

Die Grundgesamtheit der beiden repräsentativen Studien waren Lehrpersonen und Schulleitungen (inkl. Stellvertretungen) an allgemeinbildenden Schulen in ganz Deutschland. Auswahl und Rekrutierung erfolgten telefonisch im Rahmen bevölkerungsrepräsentativer Mehrthemenumfragen. Zur Teilnahme bereite Personen erhielten unmittelbar per E-Mail eine Einladung zur Online-Befragung. Im Rekrutierungsverfahren hatte jedes Mitglied der Grundgesamtheit die gleiche statistische Chance, in die Stichprobe aufgenommen zu werden. Kleinere Abweichungen der Struktur der Stichprobe von der Grundgesamtheit wurden durch Gewichtung (iteratives Verfahren) angeglichen und entsprechende Gewichtungsfaktoren ermittelt. Grundlage hierfür waren Daten des Statistischen Bundesamtes für Lehrkräfte im Schuljahr 2018/2019 an allgemeinbildenden Schulen, die für die Ziel-Verteilung der Stichprobe die Merkmale Alter, Geschlecht, Region (Ost/West) und Schulform vorgaben.

In Summe wurden 1.636 Lehrpersonen in vier in sich repräsentativen Rotationen (Zufallssplits) befragt. Hiervon bilden $n=405$ Lehrpersonen (Befragung: September bis November 2019) die hier analysierte Stichprobe, welche kontrastiert wird mit der Stichprobe von $n=405$ Schulleitungen oder deren Stellvertretungen (Befragung: August bis November 2019). Die Beschreibung der beiden Stichproben in Tabelle 1 auf der folgenden Seite weist die Verteilung nach Alter, Geschlecht, Berufserfahrung, Migrationshintergrund und Schulform aus. 
Colin Cramer, Jana Groß Ophoff, Marcus Pietsch \& Pierre Tulowitzki

Tab. 1: Stichprobenmerkmale der Lehrpersonen und Schulleitungen (in \%)

\begin{tabular}{|c|c|c|c|}
\hline Variable & Kategorie & Lehrpersonen & Schulleitungen \\
\hline \multirow[t]{4}{*}{ Alter } & bis 29 Jahre & 4.7 & 0.0 \\
\hline & 30-44 Jahre & 36.8 & 11.6 \\
\hline & 45-59 Jahre & 42.7 & 56.0 \\
\hline & 60 und mehr Jahre & 15.8 & 32.2 \\
\hline \multirow[t]{2}{*}{ Geschlecht } & weiblich & 65.2 & 55.8 \\
\hline & männlich & 34.8 & 43.7 \\
\hline \multirow{6}{*}{$\begin{array}{l}\text { Berufs- } \\
\text { erfahrung }\end{array}$} & bis 1 Jahr & 1.0 & 0.0 \\
\hline & 1-5 Jahre & 12.3 & 0.7 \\
\hline & 6-10 Jahre & 16.3 & 12.8 \\
\hline & 11-20 Jahre & 33.1 & 28.9 \\
\hline & 20 und mehr Jahre & 35.1 & 64.4 \\
\hline & unsicher bzw. keine Angabe & 2.2 & 1.0 \\
\hline Migration & Migrationshintergrund & 4.7 & 4.2 \\
\hline \multirow[t]{8}{*}{ Schulform } & Grundschule & 23.7 & 51.9 \\
\hline & Hauptschule & 5.7 & 3.0 \\
\hline & Realschule & 11.0 & 9.1 \\
\hline & (Berufliches) Gymnasium & 30.7 & 14.8 \\
\hline & Integrierte Gesamtschule & - & 3.2 \\
\hline & $\begin{array}{r}\text { Schule mit mehreren Bildungsgängen } \\
\text { (z.B. Stadtteilschule oder Gemeinschaftsschule) }\end{array}$ & 19.5 & 8.1 \\
\hline & Sonder- und Förderschule & 8.5 & 6.4 \\
\hline & andere Schulform & 1.0 & 3.5 \\
\hline
\end{tabular}

Anm.: Lehrpersonen: $n=405$; Schulleitungen: $n=405$. Eine (formale) Leitungsposition hatten die Schulleitungen im Schnitt bereits $10.1(S D=7.4)$ Jahre inne; an der spezifischen Schule arbeiteten sie im Mittel bereits $8.7(S D=6.9)$ Jahre.

Quelle: eigene Berechnung

\subsection{Erhebungsinstrumente und Datenanalyse}

Die Online-Fragebögen bestanden aus geschlossenen Fragen, die neben thematisch relevanten Skalen und Items auch demografische Angaben (u.a. Alter, Geschlecht, Schulform, Dienstalter) umfassten und zur Vermeidung von Reihenfolgeeffekten in zufälliger Reihenfolge innerhalb einer Rotation angezeigt wurden. Hierbei wurden überwiegend etablierte Skalen (s. u.) eingesetzt, die durch forsa einem Pretest im Feld unterzogen wurden (je nach Rotation 20-28 Lehrpersonen; 25 Schulleitungen). Die deskriptiven Statistiken wurden mit der Software SPSS 26 berechnet. 
In der Befragung der Lehrpersonen wurde zunächst die Affinität, eine Schulleitungsstelle zu übernehmen, über ein kategoriales Single-Item erhoben: „Haben Sie jemals konkret darüber nachgedacht, sich auf eine Stelle als Schulleiterin oder Schulleiter zu bewerben?" (Antwort: ja, nein, keine Angabe). Weiterhin wurde eine Skala entwickelt zu Gründen, warum sich Lehrpersonen nicht auf eine Schulleitungsstelle bewerben bzw. nicht Mitglied der Schulleitung sind (vgl. Kap. 4.1). Grundlage hierfür war eine Masterarbeit, in welcher Lehrpersonen hierzu interviewt wurden (Nebel, 2016). Aus den Ergebnissen wurden zehn Items abgeleitet, die typische Hindernisgründe (Unattraktivität Schulleitung) operationalisieren. Mittels explorativer Faktorenanalysen (Mplus Version 8; Muthén \& Muthén, 2017; vgl. die Anmerkungen zu Tab. 2 auf S. 139) wurden Modelle unterschiedlicher Dimensionalität über etablierte Goodness-of-Fit-Indizes verglichen (Moosbrugger \& Schermelleh-Engel, 2012). Dabei erwies sich eine dreidimensionale Lösung als die passendste (vgl. Tab. 2), wobei von ursprünglich zehn Items zwei aufgrund keiner eindeutigen Faktorzugehörigkeit bzw. einer Mindestladung $\lambda_{\mathrm{ij}} \leq .30$ auf allen Faktoren ausgeschlossen wurden (Comrey \& Lee, 1992).

Bei der Befragung der Schulleitungen wurden deren Karrieremotive für das Anstreben einer (formalen) Schulleitungsposition mithilfe einer Adaption des revidierten Work Values Inventory (Super, 1970; Zytowski, 2006; Robinson \& Betz, 2008) erhoben. $\mathrm{Zu}$ zehn der dort enthaltenen zwölf Dimensionen wurde je ein Item zur Erhebung der folgenden Bereiche eingesetzt: geistige Herausforderung, Leistung, Kreativität, Altruismus, Abwechslung, Führung Anderer, Sicherheit des Arbeitsplatzes, Unabhängigkeit, Berufsprestige und Einkommen. Ergänzt wurde dies durch eine Frage zu den antizipierten Karrierechancen: „Ich wurde Schulleitung, um meine beruflichen Aufstiegsmöglichkeiten zu verbessern." (vgl. die Anmerkungen zu Abb. 1). Zur Erfassung der Arbeitsplatzwechselabsicht wurde ein Item aus dem US-amerikanischen Schools and Staffing Survey (Boyce \& Bowers, 2016) übernommen: „Wie lange planen Sie, Schulleiterin/Schulleiter an dieser Schule zu bleiben?" (vgl. die Anmerkungen zu Abb. 2). Sofern die Befragten einen Arbeitsplatzwechsel in Erwägung zogen, wurden sie zudem mit Items aus der Studie Berufliche Laufbahnentwicklung von Akademikerinnen und Akademikern der Universität Erlangen-Nürnberg (Abele \& Spurk, 2006) sowie mit zwei ergänzten Items (altersbedingte und gesundheitliche Gründe) nach Gründen für den anvisierten Stellenwechsel befragt (Mehrfachauswahl: nein / ja).

\section{Ergebnisse}

Im Folgenden werden die zentralen Ergebnisse zu Schulleitung in Deutschland mit Blick auf die Aussagen der bevölkerungsrepräsentativen Samples von Lehrpersonen (vgl. Kap. 4.1) sowie Schulleitungen (vgl. Kap. 4.2) dargestellt. 


\subsection{Was Schulleitung für Lehrpersonen unattraktiv macht}

Ein knappes Drittel der Lehrpersonen in Deutschland (30.1\%) hat entsprechend der Angaben in der Befragung bereits konkret darüber nachgedacht, sich auf eine Stelle in der Schulleitung zu bewerben. Empirisch lassen sich drei Dimensionen (vgl. Tab. 2 auf der folgenden Seite) unterscheiden, die das Amt der Schulleitung potenziell unattraktiv machen, nämlich mit dem Amt unvereinbare, andere (pädagogische) Interessen (Dimension 1), Unsicherheit und mangelnde Vorbereitung auf die mit Schulleitung verbundenen Aufgaben (Dimension 2) sowie die erwartete Beanspruchung im Amt und seine geringen Anreize (Dimension 3). Das Modell zeigt gemäß dem Comparative Fit Index (CFI) eine gute, nach dem Root Mean Square Error of Approximation (RMSEA) eine akzeptable Modellpassung. Trotz der vergleichsweise niedrigen Faktorladung (vgl. Kap. 3) des Items „Ich empfinde die formalen Anreize als zu gering [...]" auf dem zugehörigen Faktor wurde es angesichts der inhaltlichen Passung beibehalten. Die Skalen weisen eine für Gruppenvergleiche akzeptable interne Konsistenz auf (Rost, 2013), und es ergeben sich keine signifikanten Schulformunterschiede.

Gegen das Amt der Schulleitung sprechen demnach vor allem die erwartete Beanspruchung und die geringen Anreize, die ihm zugeschrieben werden. Aufgabenfülle und Arbeitsbelastung werden als zu hoch angesehen. Die Rahmenbedingungen der Amtsausübung werden als schwierig erachtet, obwohl formalen Anreizen (z. B. Gehaltssteigerung) vergleichsweise weniger Bedeutung beigemessen wird. Die sich überschneidenden Konfidenzintervalle der Mittelwerte lassen den Schluss zu, dass die Lehrpersonen mit dem Amt der Schulleitung unvereinbare (pädagogische) Interessen als ähnlich bedeutsam erachten. Dagegen scheinen Unsicherheit und mangelnde Vorbereitung auf Aufgaben von Schulleitungen signifikant weniger ins Gewicht zu fallen: Lehrpersonen sind der Auffassung, Aufgaben von Schulleitung recht gut einschätzen zu können. Eher noch sehen sie in der mangelnden Vorbereitung auf die Aufgaben einen Grund, sich nicht für das Amt zu interessieren.

\subsection{Karrieremotive und Arbeitsplatzwechselabsichten von Schulleitungen}

Die zentralen Karrieremotive von Schulleitungen sind in der Retrospektive vor allem der Wunsch, neue Ideen entwickeln und erproben (93\%), eine abwechslungsreiche und kreative Tätigkeit ausüben ( $86 \%$ ), für das Wohl anderer Menschen sorgen (85 \%) sowie eigene Entscheidungen treffen zu wollen (85 \%) (vgl. Abb. 1 auf S. 140). Auch wollen 81 Prozent der befragten Schulleiter*innen anderen Menschen beistehen und helfen. Demgegenüber artikulieren nur 22 Prozent der Schulleitungen, dass ein (sehr) wichtiges Motiv für ihre Berufswahl gewesen sei, ihr berufliches Ansehen verbessern zu wollen, und 27 Prozent gaben an, so ihre weiteren Aufstiegsmöglichkeiten zu erhöhen. Die Steigerung des Einkommens spielte für 36 Prozent der Befragten 
Tab. 2: Gründe von Lehrpersonen gegen die Entscheidung für die Schulleitung

\begin{tabular}{lrrrrr}
\hline Dimension 1: Andere (pädagogische) Interessen & & & & & \\
\cline { 2 - 5 } Item & $M$ & $S D$ & $\lambda_{1}$ & $\lambda_{2}$ & $\lambda_{3}$ \\
\hline $\begin{array}{l}\text { Ich habe berufliche Interessen, die sich nicht mit Schullei- } \\
\text { tung verbinden lassen (z. B. primäres Interesse am Unter- } \\
\text { richten). }\end{array}$ & 4.8 & 2.1 & $\mathbf{0 . 9 2}$ & -0.00 & -0.11 \\
\hline $\begin{array}{l}\text { Ich habe kein Interesse an zentralen Aufgaben der Schul- } \\
\text { leitung (z. B. Verwaltung, Personalführung, Konfliktmanage- } \\
\text { ment). }\end{array}$ & 4.2 & 2.2 & $\mathbf{0 . 5 6}$ & 0.01 & 0.10 \\
\hline $\begin{array}{l}\text { Ich bin mit meiner Tätigkeit als Lehrperson zufrieden (z. B. } \\
\text { Zufriedenheit mit dem Unterrichten und Arbeitsumfeld). }\end{array}$ & 5.6 & 1.5 & $\mathbf{0 . 5 4}$ & $\mathbf{- 0 . 1 5}$ & 0.01 \\
\hline Skala gesamt (a $=.70)$ & 4.8 & 1.6 & (95\%-KI: 4.7-5.0) \\
\hline
\end{tabular}

Dimension 2: Unsicherheit und mangelnde Vorbereitung auf Aufgaben

\begin{tabular}{lllllll}
\hline Ich bin unsicher, welche Aufgaben sich letztlich mit Schullei- & 3.0 & 1.8 & -0.02 & $\mathbf{0 . 8 9}$ & -0.01
\end{tabular} tung verbinden (z. B. unzureichende Kenntnis der Abläufe).

Ich erachte mich nicht ausreichend für die Aufgaben der Schulleitung vorbereitet (z. B. keine hinreichende Qualifikation).

\begin{tabular}{llll}
\hline Skala gesamt $(a=.65)$ & 3.5 & 1.7 & (95\%-KI: 3.4-3.7)
\end{tabular}

Dimension 3: Erwartete Beanspruchung und geringe Anreize

$\begin{array}{lllllll}\text { Ich erachte die Aufgabenfülle und Arbeitsbelastung von } & & 5.6 & 1.8 & -0.03 & -0.02 & \mathbf{1 . 0 3}\end{array}$ Schulleitungen als zu hoch (z. B. hohe zeitliche Belastung, große Verantwortung, Stress).

Ich sehe die Rahmenbedingungen der Schulleitung als $\begin{array}{lllll}5.4 & 1.7 & 0.22 & 0.09 & \mathbf{0 . 4 6}\end{array}$ schwierig an (z. B. enge Vorgaben aus der Schuladministration).

Ich empfinde die formalen Anreize als zu gering (z. B. Gehaltssteigerung ist zu klein).

$\begin{array}{lllll}4.1 & 2.1 & 0.08 & \mathbf{0 . 5 2} & 0.07\end{array}$

Skala gesamt $(a=.65)$

$5.2 \quad 1.5 \quad(95 \%-K I: 5.1-5.4)$

Anm.: Frage: Inwieweit treffen die folgenden Gründe auf die Tatsache zu, dass Sie sich bislang nicht auf eine Stelle als Schulleiterin oder Schulleiter beworben haben bzw. nicht der Schulleitung angehören? Antwortformat: siebenstufige Skala mit Polbeschriftung ( $1=$ trifft überhaupt nicht zu; $7=$ trifft voll und ganz $\mathrm{zu}$ ). Anmerkungen: $N_{\min }=386$; $M=$ Arithmetisches $\quad$ Mittel; $\quad S D=$ Standardabweichung; $\lambda=$ Faktorladung; $95 \%$ $K I=95 \%$-Konfidenzintervall Mittelwert. Faktorenanalyse: Extraktionsmethode: Maximum Likelihood; Rotationsmethode: Geomin; $\mathrm{Chi}^{2}=18.09 ; \mathrm{df}=7 ; \mathrm{Chi}^{2} / \mathrm{df}=2.6 ; \mathrm{RMSEA}^{2}=$ .063 ; CFI $=.982$.

Quelle: eigene Berechnung

eine wichtige oder sehr wichtige Rolle bei der Entscheidung. Während aus Sicht von Schulleitungen insbesondere intrinsische und autonomiebezogene Motive genannt werden, scheinen extrinsische Motive eine vergleichsweise nebengeordnete Rolle zu spielen. 
Abb. 1: Die fünf wichtigsten Motive von Schulleitungen, sich für den Karriereschritt entschieden zu haben

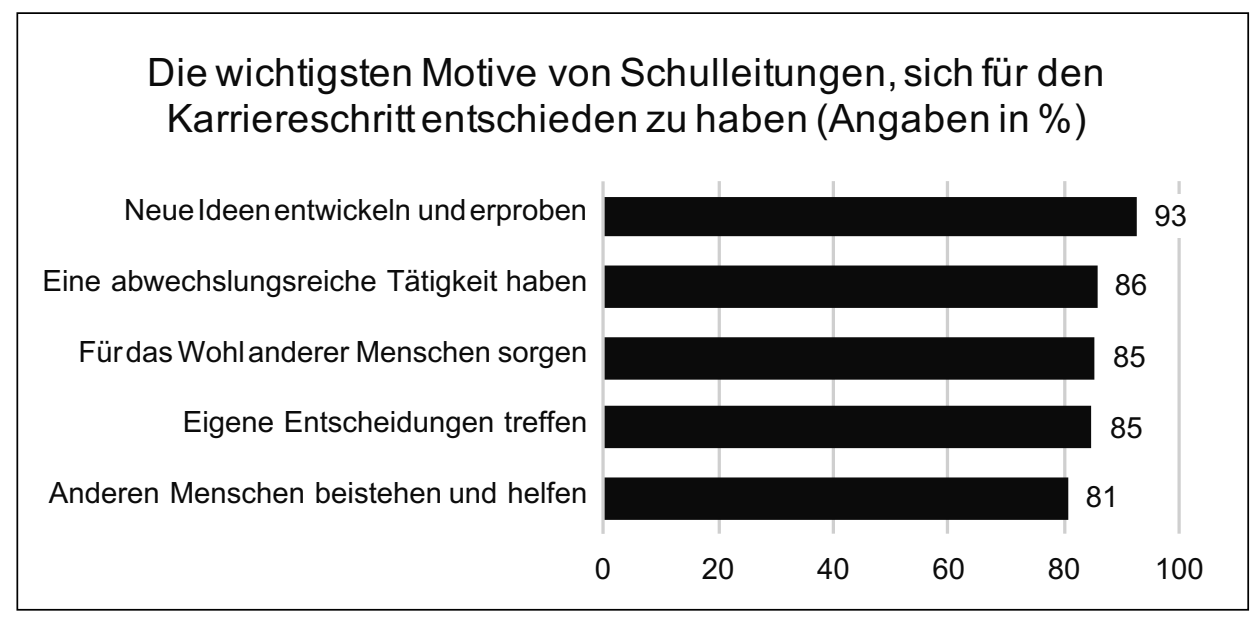

Anm.: Frage: Wie wichtig war der jeweilige Grund für Ihre Entscheidung, Schulleiterin oder Schulleiter zu werden? Antwortformat: Die prozentuale Zustimmung wurde als Summe über die Antwortstufen 3 und 4 gebildet: 1 = sehr unwichtig, $2=$ eher unwichtig, $3=$ eher wichtig, $4=$ sehr wichtig.

Quelle: eigene Darstellung

Mit Blick auf die Arbeitsplatzwechselabsichten von Schulleitungen zeigt sich, dass die befragten Schulleitungen vor Besetzung der aktuellen Stelle im Mittel 2,8mal $(S D=1,91)$ die Stelle gewechselt haben. Dabei beabsichtigt der überwiegende Anteil (67\%), auf der jetzigen Stelle so lange wie möglich zu bleiben. Dennoch planen 15 Prozent der Schulleitungen, die aktuelle Schule zu verlassen, sobald sich eine bessere Möglichkeit ergibt. Vier Prozent geben an, ihren aktuellen Arbeitsplatz so schnell wie möglich verlassen zu wollen. 13 Prozent der Befragten haben hierzu keine Auskunft gegeben. Somit beabsichtigt etwa jede fünfte Schulleitung in Deutschland, (in Zukunft) ihren Arbeitsplatz zu wechseln. Ob für diese Personen eine Schulleitungsposition an einer anderen Schule in den Blick gerät oder ob sie nicht mehr als Schulleiter*in arbeiten wollen, kann auf Basis der Daten nicht differenziert werden. Der Wechselwille variiert zwischen Schulformen (vgl. Tab. 3 auf der folgenden Seite): Schulleitungen an integrierten Gesamtschulen haben eine 71 Prozent geringere Chance, ihren Arbeitsplatz zu wechseln $(O R=0.29)$ als Schulleitungen aller anderen Schulformen. Die Schulleitungen an Hauptschulen hingegen haben eine deutlich stärker ausgeprägte Absicht, ihren Arbeitsplatz zu wechseln, als Schulleitungen an anderen Schulformen $(O R=1.86)$. 
Tab. 3: Arbeitsplatzwechselabsicht nach Schulform

\begin{tabular}{lcc}
\hline Schulform & Wechselabsicht (in \%) & Odds Ratio (OR) \\
\hline Grundschule & 22.4 & 1.17 \\
Hauptschule & 36.4 & 1.86 \\
Realschule & 26.7 & 1.13 \\
(Berufliches) Gymnasium & 16.7 & 0.74 \\
Integrierte Gesamtschule & 7.7 & 0.29 \\
Schule mit mehreren Bildungsgängen & 21.2 & 0.94 \\
(z. B. Stadtteilschule oder Gemeinschaftsschule) & & \\
\hline
\end{tabular}

Anm.: Odds Ratio (OR) geben an, wie hoch die Chance von Schulleitungen einer bestimmten Schulform ist, im Vergleich zu Schulleitungen aller anderen Schulformen ihren aktuellen Arbeitsplatz verlassen zu wollen. Ein Wert von $O R=1$ zeigt keinen Unterschied zwischen den Gruppen, bei $O R>1$ ist die Chance der Arbeitsplatzwechselabsicht größer, bei $O R<1$ geringer.

Quelle: eigene Berechnung

Wird nach Gründen für die Arbeitsplatzwechselabsicht gefragt (vgl. Abb. 2 auf der folgenden Seite), so verweisen wechselwillige Schulleiter ${ }^{\star}$ innen insbesondere auf schwierige Kontextbedingungen. Der zentrale Grund für einen beabsichtigten Arbeitsplatzwechsel ist der Wunsch nach beruflicher Entwicklung (52\%), was etwa jeder zehnten aller Schulleitungen in Deutschland entspricht. 44 Prozent der wechselwilligen Schulleitungen nennen eine nicht angemessene Bezahlung als Motiv, 31 Prozent beklagen zu wenig Unterstützung. 25 Prozent der Schulleitungen nennen Überforderung durch die Arbeitsbedingungen als Motiv der Wechselabsicht, und 24 Prozent weisen auf allgemein schlechte Arbeitsbedingungen hin, während nur zwölf Prozent der Befragten ein generell unbefriedigendes Aufgabenfeld nennen. Der Wunsch nach mehr Zeit für die Familie (19\%) sowie gesundheitliche (15\%) und altersbedingte Gründe (15\%) spielen hingegen eine geringere Rolle. Vergleichsweise selten werden auch Gründe wie ungünstige Arbeitszeiten (7 \%), das Arbeitsklima vor Ort (5\%) oder ein ungünstiger Standort der Schule (5\%) genannt. 
Abb. 2: Die fünf wichtigsten Motive von Schulleitungen, den aktuellen Arbeitsplatz wechseln $\mathrm{zu}$ wollen

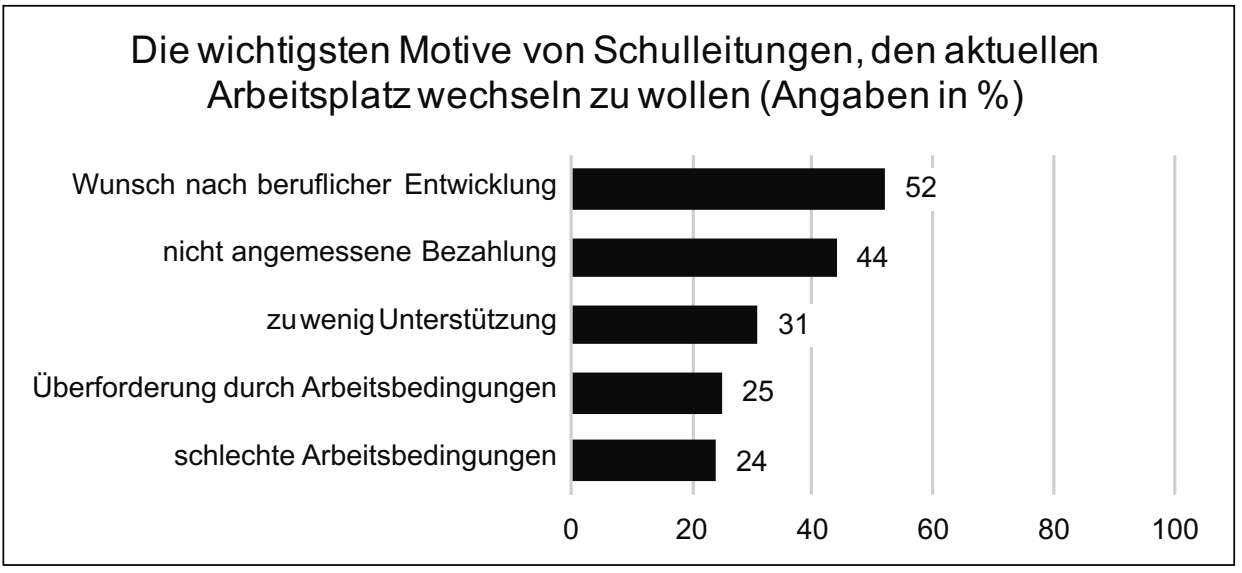

Anm.: Frage: Wie lange planen Sie, Schulleiterin/Schulleiter an dieser Schule zu bleiben? Antwortformat: Die prozentuale Zustimmung wurde als Summe über die Antwortstufen 2 und 3 gebildet: 1 = so lange, wie möglich, 2 = so lange, bis sich mir eine bessere Möglichkeit bietet, 3 = Ich plane definitiv, die Schule so schnell wie möglich zu verlassen, $4=$ kann ich nicht sagen.

Quelle: eigene Darstellung

\section{Diskussion}

Die Befragung von Lehrpersonen konnte zeigen, dass nur ein knappes Drittel bereits einmal konkret darüber nachgedacht hat, Schulleiter*in zu werden. Die wichtigsten Gründe gegen eine Schulleitungskarriere sind insbesondere die mit dem Amt verbundene erwartete hohe Arbeitsbelastung und Aufgabenfülle sowie die Zufriedenheit mit der (Unterrichts-)Tätigkeit als Lehrperson. Auch werden die Rahmenbedingungen, unter denen Schulleitungen arbeiten, als problematisch angesehen. Fehlende (finanzielle) Anreize spielen eine, wenn auch nachgeordnete Rolle.

Der Befragung von Schulleitungen zufolge erweisen sich ein Streben nach Gestaltungsmöglichkeiten, nach Verantwortung für andere und nach Autonomie in der Berufsausübung retrospektiv als zentrale Karrieremotive, während extrinsische Anreize (z.B. ein besseres Gehalt) eine geringere Rolle spielen - was sich überwiegend mit dem eingangs dargestellten Stand der (US-)Forschung deckt. Im Vergleich zu den befragten Lehrpersonen ist für Schulleitungen (also für Lehrpersonen, die den Karriereschritt vollzogen haben) die tatsächlich hohe Belastung im Amt kein maßgeblicher Hinderungsfaktor für ihre Entscheidung. Ob sich damit die Klientel der Lehrpersonen von derjenigen der Schulleitungen auch mit Blick auf Beanspruchungserleben unterscheidet, bleibt eine offene Forschungsfrage. Wenn ein Fünftel der 
Schulleitungen - insbesondere an Hauptschulen - über einen Arbeitsplatzwechsel nachdenken, so tun sie dies insbesondere aufgrund des Wunsches nach beruflicher Weiterentwicklung sowie aufgrund von zu geringer Bezahlung und Unterstützung oder schlechten Arbeitsbedingungen; private bzw. gesundheitliche Motive oder ein generell als unbefriedigend erachtetes Aufgabenfeld fallen dagegen kaum ins Gewicht.

Angesichts dieser repräsentativen Befunde steht infrage, ob alleine die von der Bildungsadministration bislang unternommenen formalen Maßnahmen zur Behebung des Mangels an Schulleitungen (z.B. moderate Erhöhung der Bezüge, Ausbau des Fortbildungsangebots, Werbemaßnahmen) zielführend sind: Entscheidend erscheint, ob die passenden (im Sinne tätigkeitsbezogener Affinität) Lehrpersonen für die Schulleitung gewonnen werden können und inwiefern Entwicklungsmöglichkeiten gegeben sind, die sie im Amt der Schulleitung halten. Offenbar zu wenig wird bislang in Rede gestellt, dass die Beanspruchung im Amt auf Seiten von Lehrpersonen möglicherweise als $(\mathrm{zu})$ bedrohlich wahrgenommen wird und sie zugleich mit ihrer Tätigkeit in Schule und Unterricht zufrieden sind. Es erscheint daher zuvorderst notwendig zu diskutieren, wie Schulleitungen im Amt unterstützt, gefördert und entlastet werden können (z.B. Raum schaffen für Entwicklungsaktivitäten durch höhere Schulautonomie, Delegation von Verwaltungsaufgaben an neu zu schaffende Stellen, Aufwertung der Qualifizierung für Schulleitungen), um für potenziell interessierte (und geeignete) Lehrpersonen attraktiver zu sein. Auch erscheint ein frühzeitigerer Kontakt mit Themen der Leitung und Schulentwicklung auf Organisationsebene sinnvoll, beispielsweise im Lehramtsstudium im Rahmen eines Wahlmoduls, um ein Verständnis für die Organisationsebene zu schärfen und frühzeitig für die Karriereoption Schulleitung zu sensibilisieren. Schulleitungen selbst sind im Amt ebenfalls überwiegend mit ihrem Aufgabengebiet zufrieden, da die Tätigkeit an sich kaum einen Grund für eine Arbeitsplatzwechselabsicht darzustellen scheint.

Keinen Einfluss hingegen hat die Bildungsadministration auf die Berufswahlmotive von Lehrpersonen, die aus ganz spezifischen und vorwiegend pädagogisch-intrinsischen Motiven heraus ihren Beruf ergreifen (Cramer, 2016) und damit kaum einen ausreichenden Pool zur Akquise von geeigneten Schulleitungen darstellen. Solche intrinsischen Motive (wie z.B. das pädagogische Interesse) sind schon zu Beginn des Lehramtsstudiums ausgeprägt (Cramer, 2012; Franz \& Groß Ophoff, 2019), vergleichsweise stabil (Roness \& Smith, 2010; Richardson \& Watt, 2016) und - zumindest aus Sicht der Mehrheit der befragten Lehrpersonen - inkompatibel mit den Tätigkeiten von Schulleitungen. Während der Fit-Choice-Theorie zufolge die Berufswahl von Lehrpersonen ein komplexes Zusammenspiel der Bewertung beruflicher Anforderungen und Erträge unter Einbezug der eigenen Kompetenzerwartung ist (Watt et al., 2012), spielen für Schulleitungen extrinsisch-verlegenheitsbezogene Motive retrospektiv eine geringere bzw. keine Rolle. Mentoring-Programme müssten daher eher dem Abgleich der Motivlagen dienen als zum Ziel zu haben, Lehrpersonen 
das Amt der Schulleitung „schmackhaft“ zu machen; denn sie meinen, die Aufgaben von Schulleitung zu kennen, und nehmen diese ggf. als abschreckend wahr.

Damit gerät die Frage nach einem eigenen und vom Lehramt zu unterscheidenden Berufsstand Schulleitung samt spezifischer Qualifikationswege in den Blick. Nicht beantwortet werden kann allerdings, ob solche Lehrpersonen ihre Affinität im Rahmen ihrer besonderen Aufgaben allererst entwickeln oder ob diese Affinität bereits der Anlass für die Übernahme besonderer Aufgaben ist. Nur im letzteren Fall könnte Schulleitung als ein vom Lehrer*innenberuf entkoppeltes Berufsbild verstanden werden, für das interessierte und geeignete Personen auch außerhalb des Lehrkörpers gewonnen und qualifiziert werden sollten. Plausibel erscheint es vielmehr, vielfältige Wege in den Beruf zu schaffen. Eine eigenständige Qualifizierung vor dem Hintergrund des tatsächlichen Aufgabenprofils könnte Diskrepanzen zwischen Erwartungen an die Schulleitung und dem Berufsalltag verringern. Bislang ist die Schulleitungsqualifizierung in Deutschland nach wie vor zu heterogen und unstrukturiert (Tulowitzki et al., 2019), während Schulleitungen fast vollständig aus dem Lehrpersonal akquiriert werden. Die Rolle von Schulaufsicht und Schulinspektion, die Schulleitungen unterstützen und motivieren können, ist ungeklärt (Heinrich, 2017; Tulowitzki, 2019b).

Die repräsentativen Ergebnisse der Studie zum Thema Schulleitung aus der teils komplementären Sicht von Lehrpersonen und Schulleitungen weisen besonderes Potenzial für die Qualifikationswege von Schulleitung auf. Limitationen ergeben sich u.a. daraus, dass die Karrieremotive von Schulleitungen retrospektiv erhoben wurden, womit die realen Motivlagen zum Zeitpunkt der Karriereentscheidung verzerrt erfasst sein könnten (recall bias; z. B. Schwarz, 2007). Diese Unschärfe kann durch die Befragung von Lehrpersonen zu Hinderungsgründen, eine Schulleitungsposition zu ergreifen, partiell kompensiert werden. Das eigens hierfür entwickelte Instrument zeigt zwar eine akzeptable bis gute Modellpassung, weist aber nur befriedigende interne Konsistenzen in den Subskalen auf. Es sollte daher in künftiger Forschung optimiert (z.B. Entwicklung weiterer Items) und um Aspekte wie Schulkultur und Schulentwicklung (vgl. Müller et al., 2017) erweitert werden, um die Motive von Lehrpersonen und Schulleitungen stärker aufeinander beziehen zu können. Ferner sollte untersucht werden, warum gerade Schulleitungen an Hauptschulen besonders häufig über einen Arbeitsplatzwechsel nachdenken. Solche Analysen werden möglich, weil das Projekt LineS als Längsschnitt angelegt ist. Forschungsdesiderate zeigen sich zudem hinsichtlich der Analyse homogener Subgruppen sowohl seitens Lehrpersonen als auch Schulleitungen. 


\section{Literatur und Internetquellen}

Abele, A. E., \& Spurk, D. (2006). Berufliche Laufbahnentwicklung von Akademikerinnen und Akademikern der Universität Erlangen-Nürnberg (BELA-E). Zugriff am 24.01.2020. Verfügbar unter: https://www.sozialpsychologie.phil.fau.de/files/2016/10/BELA2.pdf.

Bonsen, M. (2016). Wirksame Schulleitung. In H. Buchen \& H.-G. Rolff (Hrsg.), Professionswissen Schulleitung (4., überarb. u. erw. Aufl.) (S. 193-228). Weinheim: Beltz.

Boyce, J., \& Bowers, A. J. (2016). Principal Turnover: Are there Different Types of Principals Who Move from or Leave Their Schools? Leadership and Policy in Schools, 15 (3), 237272. http://dx.doi.org/10.1080/15700763.2015.1047033

Brauckmann, S. (2014). Ergebnisbericht im Rahmen des BMBF Forschungsschwerpunkts "Steuerung im Bildungssystem" (SteBis) geförderten Forschungsprojekts „Schulleitungshandeln zwischen erweiterten Rechten und Pflichten (SHaRP)“. Unter Mitarbeit von C. Herrmann, K. D. Hanßen, S. Böse, T. Holz \& T. Feldhoff. Berlin: DIPF.

Brauckmann, S., \& Herrmann, C. (2013). Belastungserleben von Schulleiterinnen und Schulleitern im Rahmen erweiterter schulischer Eigenständigkeit - Erste empirische Befunde aus der SHaRP-Studie. In I. van Ackeren, M. Heinrich \& F. Thiel (Hrsg.), Evidenzbasierte Steuerung im Bildungssystem? Befunde aus dem BMBF-SteBis-Verbund (DDS - Die Deutsche Schule, 12. Beiheft) (S. 172-196). Münster: Waxmann.

Comrey, A. L., \& Lee, H. B. (1992). A First Course in Factor Analysis. Hillsdale, MI: Lawrence Erlbaum.

Cramer, C. (2012). Entwicklung von Professionalität in der Lehrerbildung. Empirische Befunde $z u$ Eingangsbedingungen, Prozessmerkmalen und Ausbildungserfahrungen Lehramtsstudierender. Bad Heilbrunn: Klinkhardt.

Cramer, C. (2016). Berufswahl Lehramt: Wer entscheidet sich warum? In M. Rothland (Hrsg.), Beruf Lehrer/Lehrerin. Ein Studienbuch (S. 261-276). Münster: Waxmann.

Dadaczynski, K., \& Paulus, P. (2016). Wohlbefinden von Schulleitungen in Deutschland: Ausprägungen und Zusammenhänge mit Arbeit und Gesundheit. Prävention und Gesundheitsförderung, 11 (3), 171-176. https://doi.org/10.1007/s11553-016-0544-3

Day, C., \& Sammons, P. (2013). Successful Leadership: A Review of the International Literature. Zugriff am 23.07.2020. Verfügbar unter: https://files.eric.ed.gov/fulltext/ ED546806.pdf.

Federici, R. A., \& Skaalvik, E. M. (2012). Principal Self-Efficacy: Relations with Burnout, Job Satisfaction and Motivation to Quit. Social Psychology of Education, 15 (3), 295-320. https://doi.org/10.1007/s11218-012-9183-5

Franz, E.-K., \& Groß Ophoff, J. (2019). Kompetenzerleben Lehramtsstudierender im Semesterpraktikum. In T. Ehmke, P. Kuhl \& M. Pietsch (Hrsg.), Lehrer. Bildung. Gestalten. Beiträge zur empirischen Forschung in der Lehrerbildung (S. 116-127). Weinheim: Beltz.

Hancock, D. R., Hary, C., \& Müller, U. (2012). An Investigation of Factors Impacting the Motivation of German and US Teachers to Become School Principals. Research in Comparative and International Education, 7 (3), 352-363. https://doi.org/10.2304/ rcie.2012.7.3.352

Hancock, D. R., Müller, U., \& Wang, C. (2016). Motivating Teachers to Become School Principals in the U.S.A. and Germany. Journal of Global Research in Education and Social Science, 7 (2), 83-93.

Hancock, D. R., Müller, U., Wang, C., \& Hachen, J. (2019). Factors Influencing School Principals' Motivation to Become Principals in the U.S.A. and Germany. International Journal of Educational Research, 95, 90-96. https://doi.org/10.1016/j.ijer.2019.04.004

Heinrich, M. (2017). Zum Verhältnis von Schulinspektion und Schulaufsicht: Zwei Institutionen zwischen Ausdifferenzierung und Entdifferenzierung. In A. Paseka, M. 
Heinrich, A. Kanape \& R. Langer (Hrsg.), Schulentwicklung zwischen Steuerung und Autonomie (S. 155-174). Münster: Waxmann.

Huber, S. G., Gördel, B.-M., Kilic, S., \& Tulowitzki, P. (2016). Accountability in the German School System. In J. Easley II \& P. Tulowitzki (Hrsg.), Educational Accountability International Perspectives on Challenges and Possibilities for School Leadership (S. 165183). London: Routledge.

Huber, S. G., Wolfgramm, C., \& Kilic, S. (2013). Vorlieben und Belastungen im Schulleitungshandeln. In S. G. Huber (Hrsg.), Jahrbuch Schulleitung 2013 (S. 259-271). Köln: Carl Link.

Jong, D. D., Grundmeyer, T., \& Yankey, J. (2017). Identifying and Addressing Themes of Job Dissatisfaction for Secondary Principals. School Leadership \& Management, 37 (4), 354-371. https://doi.org/10.1080/13632434.2017.1338253

Klein, E. D., \& Tulowitzki, P. (2020). Die Fortbildung von Schulleiter*innen in Forschung und Praxis - ein Systematisierungsversuch. DDS - Die Deutsche Schule, 112 (3), 257276. https://doi.org/10.31244/dds.2020.03.02

Kramer, C., \& Bauer, L. (2019). Das Standortnetz von Grundschulen in Baden-Württemberg im Wandel - Entwicklungen und Einflussfaktoren. In D. Fickermann \& H. Weishaupt (Hrsg.), Bildungsforschung mit Daten der amtlichen Statistik (DDS - Die Deutsche Schule, 14. Beiheft) (S. 87-104). Münster: Waxmann. https://doi.org/10.31244/dds. bh.2019.14.06

Leithwood, K., Harris, A., \& Hopkins, D. (2020). Seven Strong Claims about Successful School Leadership Revisited. School Leadership \& Management, 40 (1), 5-22. https:// doi.org/10.1080/13632434.2019.1596077

Leithwood, K., \& Louis, K. S. (2012). Linking Leadership to Student Learning. San Francisco, CA: Jossey-Bass.

Meyer, A., Richter, D., Marx, A., \& Hartung-Beck, V. (2019). Welche Aufgaben haben Schulleitungen heute? Eine Analyse von Schulleitungsaufgaben im innerdeutschen Vergleich. Zeitschrift für Bildungsverwaltung, 35 (2), 23-44.

Moosbrugger, H., \& Schermelleh-Engel, K. (2012). Exploratorische (EFA) und Konfirmatorische Faktorenanalyse (CFA). In H. Moosbrugger \& A. Kelava (Hrsg.), Testtheorie und Fragebogenkonstruktion (S. 325-343). Berlin: Springer.

Müller, U., Hancock, D. R., Wang, C., \& Stricker, T. (2017). Warum fällt es so schwer, sich für das Schulleitungsamt zu entscheiden? Beruf: Schulleitung, 12 (1), 33-35.

Muthén, L. K., \& Muthén, B. O. (2017). MPlus Version 8. Los Angeles, CA: Muthén \& Muthén.

Nebel, L. (2016). Unbeliebter Chefsessel. Eine explorative Untersuchung von hemmenden Faktoren bei der Übernahme von Schulleitungspositionen. Unveröff. Masterarbeit. Tübingen: Universität Tübingen.

OECD (Organization for Economic Cooperation and Development). (2018). TALIS 2018 Results, Vol. I: Teachers and School Leaders as Lifelong Learners. Paris: OECD. https:// doi.org/10.1787/1d0bc92a-en

Pietsch, M., \& Tulowitzki, P. (2017). Disentangling School Leadership and Its Ties to Instructional Practices - an Empirical Comparison of Various Leadership Styles. School Effectiveness and School Improvement, 28 (4), 629-649. https://doi.org/10.1080 /09243453.2017.1363787

Pietsch, M., Tulowitzki, P., \& Hartig, J. (2019). Examining the Effect of Principal Turnover on Teaching Quality: A Study on Organizational Change with Repeated Classroom Observations. School Effectiveness and School Improvement, Advance Online Publication, 1-23. https://doi.org/10.1080/09243453.2019.1672759

Pont, B., Nusche, D., \& Moorman, H. (2008). Improving School Leadership, Vol. 1: Policy and Practice. Paris: OECD. 
Richardson, P. W., \& Watt, H. M. G. (2016). Factors Influencing Teaching Choice: Why Do Future Teachers Choose the Career? In J. Loughran \& M. L. Hamilton (Hrsg.), International Handbook of Teacher Education (S. 275-304). Singapore: Springer.

Robinson, C. H., \& Betz, N. E. (2008). A Psychometric Evaluation of Super's Work Values Inventory-Revised. Journal of Career Assessment, 16 (4), 456-473.

Roness, D., \& Smith, K. (2010). Stability in Motivation during Teacher Education. Journal of Education for Teaching, 36 (2), 169-185. https://doi/10.1080/02607471003651706

Rost, D. H. (2013). Interpretation und Bewertung pädagogisch-psychologischer Studien. Eine Einführung (3. Aufl.). Bad Heilbrunn: Klinkhardt.

Ryan, R. M., \& Deci, E. L. (2017). Self-Determination Theory. Basic Psychological Needs in Motivation, Development, and Wellness. New York: Guilford.

Sadigh, P., \& dpa (2019, 15. Dezember). Schulleiter: Bundesweit fehlen Rektoren in den Schulen. ZEIT online. Zugriff am 06.02.2020. Verfügbar unter: https://www.zeit.de/ge sellschaft/schule/2019-12/schulleiter-mangel-schule-grundschule.

Schwanenberg, J., Klein, E. D., \& Walpuski, M. (2018). Wie erfolgreich fühlen sich Schulleitungen und welche Unterstützungsbedürfnisse haben sie? (SHIP Working Paper Reihe, No. 03). Essen: Universität Duisburg-Essen. https://doi.org/10.17185/duepublico/ 47202

Schwarz, N. (2007). Retrospective and Concurrent Self Reports. The Rationale for Real-time Data Capture. In A. A. Stone, S. Shiffman, A. A. Atienza \& L. Nebeling (Hrsg.), The Science of Real-time Data Capture (S. 11-26). New York: Oxford University Press.

Steiger, J. H. (1990). Structural Model Evaluation and Modification: An Interval Estimation Approach. Multivariate Behavioral Research, 25 (2), 173-180. http://dx.doi.org/10.1207/ s15327906mbr2502_4

Super, D. E. (1970). Work Values Inventory. Boston, MA: Houghton Mifflin.

Tekleselassie, A. A., \& Villarreal, P. I. (2010). Career Mobility and Departure Intentions among School Principals in the United States: Incentives and Disincentives. Leadership and Policy in Schools, 10 (3), 251-293.

Torres, A. C. (2018). Push, Pull, Tap and Switch: Understanding the Career Decisions of Charter School Leaders. Leadership and Policy in Schools, 19 (2), 171-189. https://doi. org/10.1080/15700763.2018.1513155

Tran, H. (2017). The Impact of Pay Satisfaction and School Achievement on High School Principals' Turnover Intentions. Educational Management Administration \& Leadership, 45 (4), 621-638. https://doi.org/10.1177/1741143216636115

Tulowitzki, P. (2019a). School Leader Recruitment, Retention and Motivation in Europe. Desk-Research-Bericht für das European Educational Policy Network (EEPN). http:// dx.doi.org/10.26041/fhnw-2034

Tulowitzki, P. (2019b). Supporting Instructional Leadership and School Improvement? Reflections on School Supervision from a German Perspective. Journal of Educational Administration, 57 (5), 571-581. https://doi.org/10.1108/JEA-03-2019-0040

Tulowitzki, P., Hinzen, I., \& Roller, M. (2019). Die Qualifizierung von Schulleiterinnen und Schulleitern in Deutschland - ein bundesweiter Überblick. DDS - Die Deutsche Schule, 111 (2), 149-170. https://doi.org/10.31244/dds.2019.02.04

Wang, F., Pollock, K., \& Hauseman, C. (2018). School Principals' Job Satisfaction: The Effects of Work Intensification. Canadian Journal of Educational Administration and Policy, 185, 73-90.

Warwas, J. (2012). Berufliches Selbstverständnis, Beanspruchung und Bewältigung in der Schulleitung. Wiesbaden: VS.

Watt, H. M. G., Richardson, P. W., Klusmann, U., Kunter, M., Beyer, B., Trautwein, U., et al. (2012). Motivations for Choosing Teaching as a Career: An International Comparison 
Colin Cramer, Jana Groß Ophoff, Marcus Pietsch \& Pierre Tulowitzki

Using the FIT-Choice Scale. Teaching and Teacher Education, 28 (6), 791-805. http:// dx.doi.org/10.1016/j.tat

Wissinger, J. (1996). Perspektiven schulischen Führungshandelns. Eine Untersuchung über das Selbstverständnis von SchulleiterInnen. Weinheim: Juventa.

Zytowski, D. (2006). Super Work Values Inventory-Revised: Technical Manual (Version 1.0). Zugriff am 01.07.2020. Verfügbar unter: https://www.kuder.com/research/technicalbriefs/supers-work-values-inventory-r/.

Colin Cramer, Prof. Dr., geb. 1979, Lehrstuhlinhaber für Professionsforschung unter besonderer Berücksichtigung der Fachdidaktiken an der Eberhard Karls Universität Tübingen.

E-Mail: colin.cramer@uni-tuebingen.de

Korrespondenzadresse: Eberhard Karls Universität Tübingen, Wilhelmstraße 31, 72074 Tübingen

Jana Groß Ophoff, Prof. Dr., geb. 1976, Professorin für Bildungswissenschaften an der Pädagogischen Hochschule Vorarlberg.

E-Mail: jana.grossophoff@ph-vorarlberg.ac.at

Korrespondenzadresse: Pädagogische Hochschule Vorarlberg, Liechtensteiner Straße 33-37, 6800 Feldkirch, Österreich

Marcus Pietsch, PD Dr., geb. 1974, Professur für Empirische Grundschulpädagogik an der Leuphana Universität Lüneburg.

E-Mail: marcus.pietsch@leuphana.de

Korrespondenzadresse: Leuphana Universität Lüneburg, Universitätsallee 1, 21335 Lüneburg

Pierre Tulowitzki, Prof. Dr., geb. 1982, Leiter der Professur für Bildungsmanagement und Schulentwicklung an der Pädagogischen Hochschule der Fachhochschule Nordwestschweiz.

E-Mail: pierre.tulowitzki@fhnw.ch

Korrespondenzadresse: Pädagogische Hochschule der Fachhochschule Nordwestschweiz, Bahnhofstrasse 6, 5210 Windisch, Schweiz 


\title{
BERICHTE ZUM SCHWERPUNKTTHEMA
}

DDS - Die Deutsche Schule

113. Jahrgang 2021, Heft 2, S. 149-160

https://doi.org/10.31244/dds.2021.02.03

CC BY-NC-ND 4.0 Waxmann 2021

Stephan Gerhard Huber \& Nadine Schneider

\section{Durchgängige und systematische schulische Führungskräftegewinnung und -entwicklung}

\section{Zusammenfassung}

Das Spektrum des Handelns von Schulleitung und Schulaufsicht als pädagogische Führungskräfte im schulischen Personalmanagement ist vielschichtig, kann mehrebenenanalytisch betrachtet werden, ist direkt und indirekt: Zum einen qualifizieren sich pädagogische Führungskräfte selbst weiter und zum anderen initiieren, begleiten und unterstützen sie die Gewinnung und Professionalisierung von schulischen Akteur*innen bzw. Nachwuchsführungskräften. Ausgerichtet ist schulisches Personalmanagement auf die Sicherung und Weiterentwicklung der Qualität pädagogischer Arbeit. In dieser doppelten Perspektive wird über ein Kooperationsprojekt der Stiftung der Deutschen Wirtschaft (sdw), der Robert Bosch Stiftung (RBS) und des Instituts für Bildungsmanagement und Bildungsökonomie (IBB) der Pädagogischen Hochschule Zug/Schweiz berichtet, das impulsgebend sein könnte für eine schulische Führungskräfteentwicklung als systematischer und kontinuierlicher berufsphasenübergreifender Prozess, der in der Lehrer*innenbildung beginnen sollte.

Schlüsselwörter: Pädagogische Führung, Schulleitung, Schulaufsicht, Führungskräfteentwicklung, systematischer Prozess, kontinuierlicher Prozess, Personalentwicklung, Personalmarketing

\section{Consistent and Systematic Recruitment and Development of School Leaders}

\begin{abstract}
Being educational leaders in the school system, the scope of action of school leadership and school administration concerning human resource management is multi-layered, can be analyzed from a multi-level perspective, is direct and indirect: On the one hand, educational leaders care for their own professional development, and on the other hand they initiate, accompany, and support the recruitment and professionalization of school actors or teachers aspiring leadership. Human resource management within
\end{abstract}


the school system is aimed at ensuring and further developing the quality of educational work. Along this double perspective, this paper focuses on a cooperation project of the Foundation of the German Economy ( $s d w)$, the Robert Bosch Foundation (RBS) and the Institute for the Management and Economics of Education (IBB) at the University of Teacher Education Zug (PH Zug), Switzerland, which could give impulses for school leadership development as a systematic and continuous process.

Keywords: educational leadership, school leadership, school administration, school supervising authorities, school inspectorate, leadership development, systematic process, continuous process, personnel development, personnel marketing.

\section{Einleitung}

Schulleitung und Schulaufsicht sind pädagogische Führungsfunktionen, deren Vertreter ${ }^{\star}$ innen auf unterschiedlichen Hierarchieebenen des Schulsystems agieren: Die Schulleitung trägt die Gesamtverantwortung auf der Ebene der Einzelschule und bewältigt dort vielfältige Aufgaben mit dem Ziel der Sicherung und Weiterentwicklung der Qualität schulischer Arbeit. Ihr Kerngeschäft ist die Sicherung der Qualität von Lehren und Lernen. Die Schulaufsicht ist im Sinne einer

„staatlichen Gesamtverantwortung für den Zustand und die Entwicklung des öffentlichen Schulwesens [zuständig]. Schulaufsicht ist Aufsicht, Administration, Planung, Beratung und Unterstützung zugleich“ (Rolff, 1998, S. 215).

In den letzten zwei Dekaden gab es weitreichende Veränderungen und Erweiterungen von Aufgaben und Verantwortlichkeiten im Zusammenspiel von Schulleitung und Schulaufsicht und zugleich haben sich die Steuerungslogiken verändert (vgl. u.a. Huber, 2020a, 2020b; Huber, Arnz \& Klieme, 2020; Huber, Tulowitzki \& Hameyer, 2017; Maaz, Huber, Arnz \& Klieme, 2020). Im Zuge zunehmender Dezentralisierung im Hinblick auf Führungs- und Organisationsstrukturen und der daraus folgenden Verlagerung von Aufgaben und Verantwortlichkeiten wurde der Einzelschule mehr Eigenverantwortung zugesprochen. Gleichzeitig verstärkten sich aber auch die zentrale Einflussnahme und Kontrolle, beispielsweise durch mehr Rechenschaftspflicht und Qualitätskontrollen durch externe Evaluation, die auf die Weiterentwicklung der Schule als Gesamteinheit ausgerichtet ist und somit auch unterstützende und beratende Elemente umfasst.

Infolgedessen fungiert Schulaufsicht nicht mehr nur als Kontroll- bzw. ControllingInstanz, sondern zu ihren Aufgaben gehören auch die Unterstützung, Förderung und Beratung von Schulen. In Anlehnung an Rosenbuschs (1994) Formulierung von der „strukturell gestörten Beziehung“ zwischen Lehrerinnen und Schulrät*innen sprechen Buchen und Burkard (2002, S. 51) knapp eine Dekade später „vom strukturell gestörten Verhältnis zur professionellen Arbeitsbeziehung“ in der Zusammenarbeit 
Durchgängige und systematische schulische Führungskräftegewinnung und -entwicklung |

von Schule und Schulaufsicht. Letztendlich geht es um ein umfassendes Modell des Qualitätsmanagements, das auf die stattfindenden Veränderungen ausgerichtet ist. Denn es zeigt sich,

„dass die alleinige Verschiebung von Zuständigkeiten, weg von Schulbehörden hin zu den Einzelschulen, noch kein Garant für die Verbesserung von Schule ist. Zentral ist dabei die Entwicklung der Schulverwaltung von einer linear agierenden Bürokratie hin zu einem kooperativen und bedarfsorientierten Dienstleister für Schulen“ (Rosenbusch \& Huber, 2018, S. 751).

\section{Personalentwicklung auf der Ebene der Schulaufsicht}

Die erweiterten Aufgaben der Schulaufsicht und die neuen Rollenzuschreibungen erfordern vielfältige Kompetenzen, deren Erwerb und Entwicklung für Verantwortliche der Schulaufsicht bislang noch unzureichend gegeben sind. Hajo Sassenscheidt ${ }^{1}$ spricht in einer ersten Zwischenbilanz aus der Perspektive der Aus- und Fortbildung schulischer Führungskräfte von einem Paradoxon: Während Personal- resp. Führungskräfteentwicklung für die Funktion Schulleitung im bundesdeutschen Kontext mittlerweile auf einem guten Niveau sei, sei die Führungskräfteentwicklung für schulaufsichtliche und schuladministrative Führungsfunktionen bislang kaum etabliert, und kritisch bemerkt er, dass ihm

„keine Bildungsadministration bekannt [sei], die in ihrer hausinternen PE und im behördeneigenen Qualitätsmanagement den Standards [entspreche], die sie von den Schulen routinemäßig erwartet[en]. Die Schulleitungen [seien] wesentlich besser qualifiziert als die Führungsebene über ihnen. Schulaufsicht und Bildungsadministration [hätten] somit keine Vorbild-, geschweige denn eine Vorreiterfunktion für die Personalentwicklung der Schulen“" (Sassenscheidt, 2020, S. 246).

Er empfiehlt daher Entwicklung und Umsetzung von Konzepten für die schulaufsichtliche Personalentwicklung, da die gestiegenen Qualitätserwartungen an Schule insgesamt zu einem Bedeutungszuwachs von Personalentwicklung an den Schulen selbst führten (vgl. Sassenscheidt, 2020, S. 243 unter Bezug auf Arnold, 2010, S. 79ff.). Eine impulsgebende Aufsicht über diese Schulen könne jedoch nur dann erfolgreich sein, wenn das Schulaufsichtspersonal entlang dieser Qualitätserwartungen handeln und auch sich selbst weiterentwickeln würde. Denn Personalentwicklung sei ein wichtiger „Erfolgstreiber“ (Sassenscheidt, 2020) - auch für das Personal der Schulaufsicht. Entsprechende Ansätze sind in einigen Bundesländern $\mathrm{zu}$ beobachten: Leitbilder für Schulaufsicht gibt es unter anderem in Niedersachsen, Nordrhein-Westfalen, im

$1 \mathrm{Zu}$ den beruflichen Erfahrungen von Dr. Hajo Sassenscheidt und aktuellen Tätigkeiten hinsichtlich Schul- und Organisationsberatung, Personalentwicklung, Moderation und Evaluation siehe http://hajo-sassenscheidt.de/index.php/berufliche-stationen/. 
Regierungsbezirk Oberbayern oder (für die Förderschulaufsicht) in Mittelfranken. In Nordrhein-Westfalen liegt seit längerem auch eine Aufgabenbeschreibung für die Schulaufsicht vor. In Berlin befasst sich die Projektgruppe „Qualitätsmanagement der Schulaufsicht“ seit einigen Jahren verstärkt mit der Rolle und Funktion der Schulaufsicht, jüngst beispielsweise auch im Rahmen des Projekts „School Turnaround“. Die Bundesländer Berlin und Brandenburg gehören zudem zu den ersten, die ein umfassendes und systematisches Qualifizierungsprogramm für die Verantwortlichen der Schulaufsichtsbehörden entwickelt haben, das die diesen zugeschriebene Rollenvielfalt als Berater*in (Unterstützung und Moderation), Leader (Personalentwicklung, Qualitätsentwicklung) und Manager ${ }^{\star}$ in (Steuerung, Controlling, Organisation, Verwaltung) abbildet. Sachsen-Anhalt hat seine Schulfachlichen Referent ${ }^{*}$ innen von 2017 bis 2019 in einer Fortbildungsreihe zur systemischen Organisationsberatung (nach)qualifiziert; die Maßnahme wurde von artop - einem An-Institut der Humboldt-Universität Berlin - durchgeführt, eine Qualifizierung, die der vielschichtigen, über die der Führungskraft und Entscheider*in hinausreichenden Rolle gerecht zu werden versucht und auch deren Rolle als Mutmacher ${ }^{\star}$ in, Nothelfer*in und Berater*in in den Blick genommen hat. ${ }^{2}$

Was jedoch bislang fehlt, ist ein verlässlicher, empirisch gesicherter Gesamtüberblick zum Ist-Stand der Professionalisierung von Schulaufsicht. Hilfreich hierfür wären Untersuchungen zu Aufgabenbeschreibungen und Tätigkeitsprofilen von Schulaufsicht, zu den aktuellen Anforderungen an dieselbe, zu den dafür zu erwerbenden Kompetenzen und dem sich daraus ergebenden Leitbild einer bzw. eines Verantwortlichen als pädagogische Führungskraft; notwendig wären Untersuchungen zu Rekrutierungs- und Auswahlverfahren, zur aktuellen Qualifizierungssituation von Schulaufsichtspersonal sowie zu berufsbegleitenden Unterstützungs- und Beratungsangeboten.

\section{Personalentwicklungskonzepte für Schulleitungen als Anregung für Qualifizierungsmaßnahmen der Schulaufsicht}

Die Schulleitung hat in den letzten 15 Jahren in den deutschen Bundesländern (wie auch in Österreich und der Schweiz) einen enormen Professionalisierungsschub erlebt. ${ }^{3}$ Die zu ihrer Qualifizierung und Professionalisierung entwickelten Maßnahmen

2 Weitere Informationen zur Projektgruppe „Qualitätsmanagement der Schulaufsicht“ sind zu finden in Senatsverwaltung für Bildung, Wissenschaft und Forschung (2007), zum Projekt „School Turnaround“ in Berlin in Trageser \& Huber (2020) und in Huber, Schneider \& Pruitt (2020), zu Professionalisierung der Schulaufsicht in Berlin und Brandenburg in Missal (2017) und zu Sachsen-Anhalt in Schmelzer \& Löffler (2020).

3 Zu diesem Fazit kommen die Autor*innen aufgrund der Befunde und Erfahrungen aus den empirischen Studien und wissenschaftlichen Begleitungen mit evaluativen Anteilen, die sie in den letzten zwei Jahrzehnten im Projekt-Cluster „Wirksamkeit von Fort- und Weiterbildung / Führungskräfteentwicklung - Theorie und Empirie" durchgeführt haben. Zentrale 
bieten zugleich vielfache Anregungen für die Qualifizierung der Schulaufsicht. Beispielsweise wurden vielfältige, umfangreiche Qualifizierungsprogramme entwickelt: orientierende und auf die Führungsfunktion Schulleitung vorbereitende Qualifizierungen, einführende Qualifizierungen für neu ernannte sowie berufsbegleitende Programme für erfahrene Schulleitungen. Mit Blick auf die Professionalisierung von Schulaufsicht wäre ein erster wichtiger und folgelogischer Schritt, auch für diese Akteur*innen ähnlich umfangreiche Qualifizierungsangebote zu entwickeln, auch in Anknüpfung an die für Schulleitungen etablierten Programme, und dabei beide Führungsfunktionen integriert zu denken. Dies bietet sich an, weil die Überschneidungen im Bereich der für pädagogische Führung innerhalb der Einzelschule $\mathrm{zu}$ erwerbenden Kompetenzen und der für die Schulaufsicht benötigten Kompetenzen groß sind, so dass diese Angebote sogar kooperativ bearbeitet werden könnten. Hinzu müssten speziell auf die Zielgruppe Schulaufsicht ausgerichtete Angebote kommen.

\section{Kompetenzen für pädagogische Führung in der Lehrkräftebildung}

Die Themen pädagogische Führung und Leadership sowie Entwicklungsmöglichkeiten von Lehrkräften sind mittlerweile zentrale phasenübergreifende „new topics“ auch in der Lehrkräfteausbildung. Dies lässt sich der 2017 durchgeführten länderübergreifenden Studie von Drahmann und Huber zu den damaligen Entwicklungen und Herausforderungen in allen drei Phasen der Lehrkräftebildung im deutschsprachigen Raum entnehmen. Dabei geht es nicht um eine „vorzeitige Qualifizierung für Schulleitung oder Schulaufsicht“, sondern um die auch für alle Lehrkräfte wichtige Auseinandersetzung mit Fragen rund um die Qualität von Schule als Organisation und deren Weiterentwicklung, um Fragen gelingender Kooperation der schulischen Akteur*innen innerhalb der eigenen Schule wie auch zwischen Schulen und mit anderen nichtschulischen Partner*innen. Darin eingebettet können GovernanceThemen wie Aufgaben und Rollen von Führungskräften in Schule und Schulaufsicht sowie das Zusammenwirken von Steuerungsmechanismen und Führungsfunktionen sowie Schulreformen thematisiert werden.

In dieser Perspektive ist das Kooperationsprojekt „Entwicklung von Kompetenzen für pädagogische Führung“ der Stiftung der Deutschen Wirtschaft (sdw), der Robert Bosch Stiftung (RBS) und des Instituts für Bildungsmanagement und Bildungsökonomie (IBB) der Pädagogischen Hochschule Zug/Schweiz von Interesse, ${ }^{4,5}$ in dem Ansätze für ein Konzept einer folgelogischen, stimmigen und verzahnten Qualifi-

Publikationen, insbesondere Huber, 2003, 2013, 2019, sind zu finden unter www.Bildungs management.net/FKE.

4 Für alle Informationen zum Projekt und zu den im Rahmen dessen erschienenen Publikationen siehe www.Bildungsmanagement.net/EKPF.

5 Dieser Abschnitt fußt auf der von Huber, der Stiftung der Deutschen Wirtschaft und der Robert Bosch Stiftung herausgegebenen Publikation (2015). 
zierung des pädagogischen Personals von der Lehrkräftebildung bis hin zur Ebene der schulaufsichtlichen Führungskräfte als kontinuierlichem Prozess entwickelt wurden. Unter der wissenschaftlichen Begleitung des Autors und der Autorin des vorliegenden Beitrags und unterstützt von einer Expert*innengruppe aus Wissenschaft, Schulpraxis und allen drei Phasen der Lehrkräftebildung ${ }^{6}$ wurden in diesem Projekt ein Kompetenzmodell pädagogischer Führung erarbeitet sowie Empfehlungen für eine systematische, langfristig orientierte und nachhaltige Entwicklung entsprechender Kompetenzen in den verschiedenen Phasen der Lehrkräftebildung erarbeitet. Das Kompetenzmodell und die Empfehlungen werden nachfolgend kurz vorgestellt. Darüber hinaus wurden auch eine explorative Befragung zu Beispielen hinsichtlich der Entwicklung von Kompetenzen für pädagogische Führung in der Lehrkräftebildung durchgeführt und Fallvignetten zu Situationen aus dem Arbeitsalltag von Schulleiter*innen für den Einsatz in Auswahl- (z.B. Needs-Assessment) und Qualifizierungsmaßnahmen erarbeitet. Ziel der Expert*innengruppe war es, ein Verständnis für und eine Motivation zur Mitgestaltung von Schule als immanenten Bestandteil der gesamten Lehrkräftebildung zu verankern.

Die Besonderheit dieses Kooperationsprojektes ist das erweiterte Verständnis von „pädagogischer Führung“ durch den systematischen Einbezug der drei Qualifizierungsphasen - Lehramtsstudium, Vorbereitungsdienst und Fortbildung - mit dem Ziel, dass sich Lehrkräfte über ihre Führungsaufgaben in Unterricht und Erziehung hinaus für die Qualität von Schule engagieren und ihre Schule - sei es auch nur zeitbegrenzt - (mit-)gestalten: zum Beispiel als Leitung oder Mitglied einer Steuergruppe oder Projektgruppe der Schule. Sie alle tragen im Rahmen des Schulmanagements und der Schulentwicklung zur Weiterentwicklung der Schulqualität bei, und dies erfordert Kompetenzen in pädagogischer Führung. In dieses Kontinuum könnte und sollte auch die Schulaufsicht einbezogen werden.

Das im Kooperationsprojekt entwickelte Kompetenzstrukturmodell für pädagogische Führung kann als Ausgangspunkt für die Planung, Konzeption und Durchführung von kompetenzorientierten Qualifizierungsmaßnahmen für die Entwicklung von Kompetenzen für pädagogische Führung auf allen Ebenen des Schulsystems dienen wie auch als Orientierung und Anhaltspunkt für die Forschung und Evaluation (vgl. Abb. 1; vgl. Huber, Schneider, Gleibs \& Schwander, 2013; Frey \& Jung, 2011). Ausgangspunkt sind tätigkeitsbezogene Kompetenzen auf Basis von Berufsanforderungen auf verschiedenen Ebenen der Führung bzw. in Bezug auf ver-

6 Die Mitglieder der Expert ${ }^{*}$ innengruppe stammen aus verschiedenen deutschen Bundesländern und nehmen unterschiedliche Perspektiven auf die Lehrer*innenbildung ein: Schulverwaltung und Schulaufsicht: Torsten Klieme, Claudia Rugart; erste Phase der Lehrer*innenbildung: Heike Ekea Gleibs, Birgit Weyand; zweite Phase der Lehrer*innenbildung: Carsten Groene, Peter Koderisch; dritte Phase der Lehrer*innenbildung: Matthias Böckermann, Sibylle Engelke, Norbert Ryl, Hajo Sassenscheidt; Lehramtsstudierende und Lehramtsanwärter: Kay Adenstedt, Inéz-Maria Wellner; Wissenschaft: Martin Drahmann (†), Stephan Gerhard Huber, Nadine Schneider, Pierre Tulowitzki. 
Durchgängige und systematische schulische Führungskräftegewinnung und -entwicklung |

schiedene Funktionen. Der Darstellungsform nach bietet es eine Übersicht über die tätigkeitsübergreifenden, erfolgsrelevanten Dispositionen und erfolgsrelevanten Führungskonzepte zur Sicherung und Weiterentwicklung der Qualität von Schule durch die frühzeitige Sensibilisierung für und Übernahme von Gestaltungsaufgaben.

Abb. 1: Drei Elemente einer Kompetenzstruktur pädagogischer Führung (nach Huber et al., 2013, 2015; Huber \& Hiltmann, 2011)

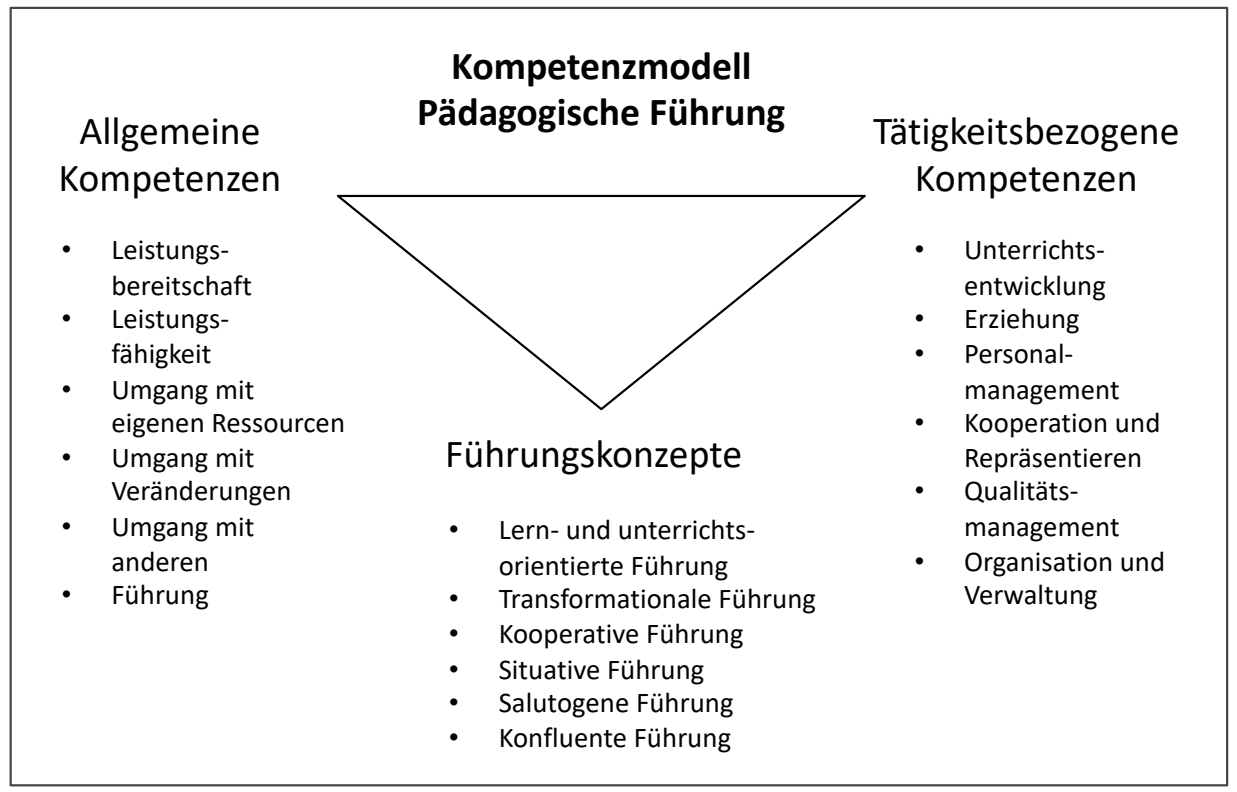

Quelle: eigene Darstellung

\section{Empfehlungen für eine systematische, langfristig orientierte und nachhaltige Entwicklung von Kompetenzen für pädagogische}

\section{Führung}

Im Rahmen des Kooperationsprojekts wurden „Empfehlungen“7 für eine systematische, langfristig orientierte und nachhaltige Entwicklung von Kompetenzen für pädagogische Führung in der gesamten Lehrkräftebildung formuliert, die hier nur überblicksartig präsentiert werden können. Sie sind einerseits darauf ausgerichtet, die eigene(n) Rolle(n) als schulische oder schulaufsichtliche Führungskraft und die daraus abzuleitenden Aufgaben zu reflektieren. Anderseits bieten sie Orientierung in Fragen der Kooperation, Beratung und Unterstützung - sowohl seitens der Zusammenarbeit von Schulaufsicht und Schulleitung als auch seitens der Schulleitung

7 „Empfehlungen“ ist der Terminus, der im Projekt verwendet wurde. Die Empfehlungen fußen teilweise auf bereits umgesetzten Konzepten an Hochschulen und Fortbildungsinstituten; ausführlicher sind sie in Huber et al. (2015) beschrieben. 
und den Lehrkräften der jeweiligen Schule. Ziel ist immer das Erleben beruflicher Wirksamkeit, die Weiterentwicklung beruflicher Kompetenzen und ein Zugewinn an Expertise. In diesem Sinne

1) ist Schule als ein Gestaltungsraum zu verstehen, der die an Bildung beteiligten Akteur*innen einbindet und ihnen vielfältige Entwicklungs- und Bewährungsfelder bietet;

2) ist in der Schule als Institution des Lernens Kooperation als Ziel und Methode zugleich zu verstehen;

3) professionalisieren sich Lehrkräfte und Führungskräfte im Gestaltungsraum Schule systematisch und nachhaltig;

4) kooperieren Verantwortliche aller drei Phasen der Lehrkräftebildung funktional und intensiv;

5) professionalisieren sich auch die Ausbilder ${ }^{*}$ innen sowie die Fort- und Weiterbildner ${ }^{*}$ innen systematisch und nachhaltig;

6) steht systematisches Personalmanagement im Zentrum von Führung und Management in Schule und Schulsystem;

7) setzen Personalmarketing und Nachwuchsförderung für Aufgaben der pädagogischen Führung frühzeitig an und werden intensiver betrieben;

8) erfolgt ein Feedback zur Förderung der Professionalisierung frühzeitig und letztere kann laufbahnbegleitend unterstützt werden;

9) werden langfristig angelegte Anreizsysteme für pädagogische Führung auf- und ausgebaut, z. B. Entlohnung, Bonussysteme, Beförderung, Gratifikation, weitere Nebenleistungen wie Versorgungsleistungen.

Auf die Empfehlungen 6 und 7 wird im Folgenden genauer eingegangen. Ausgehend von den Teilfunktionen des Human Resource Management (vgl. Liebel \& Oechsler, 1994) gehören zu einer kontinuierlichen Professionalisierung pädagogischer Führungskräfte im Sinne einer systematischen Führungskräfteentwicklung neben den impliziten Maßnahmen der Personalgewinnung und geeigneten Qualifizierungsangeboten auch explizite Maßnahmen zur kurz-, mittel- und langfristigen Nachwuchsgewinnung. Ziel ist ein systematisches Personalmarketing, eine gezielte kontextbezogene Auswahl von Führungskräften sowie deren Unterstützung und Förderung. Eine systematische Personalentwicklung inklusive Nachwuchsförderung ist Aufgabe der jeweils personalführenden Stellen - der Schulleitung mit Blick auf die an Führung und Schulmanagement interessierten Lehrkräfte in ihrer Schule und der Schulaufsicht mit Blick auf Führungskräfte in Schule. In diesem Sinne kann Personalgewinnung über (frühzeitige) Personalentwicklung gelingen. Dafür bedarf es - wie Natalie Schick (2013) im Zusammenhang mit der strategischen innerschulischen Führungskräfteentwicklung durch die Schulleitung betont - Lerngelegenheiten und ausreichend Raum und Zeit für die Erprobung einschließlich (dialogischer) Reflexion. 
Beide Führungsfunktionen sind auf die Sicherung und Weiterentwicklung der Qualität der pädagogischen Arbeit ausgerichtet. Zwar agieren sie auf unterschiedlichen Hierarchieebenen - die Schulleitung auf der Ebene der Einzelschule und die Schulaufsicht auf der Ebene des öffentlichen Schulwesens einer Region oder eines Landes -, aber als zwei Seiten einer Medaille haben sie beide die schulische Qualität im Blick. Was läge da nicht näher, als den potenziellen Nachwuchs für diese Funktionen frühzeitig mit diesen Aufgaben und deren Rolle bekannt zu machen, den ansonsten vor allem auf den Unterricht gerichteten Blick für die schulisch-organisatorischen und steuerungsdynamischen Zusammenhänge zu öffnen und den Nachwuchs für die Möglichkeiten der Mitgestaltung des eigenen Arbeitsfeldes zu gewinnen wie auch gegebenenfalls für die Übernahme entsprechender Funktionen?

In dieser Perspektive muss die Schulaufsicht, sofern sie ihrer Verantwortung für Führungskräfteentwicklung gerecht werden will, eine doppelte Perspektive einnehmen: auf die eigene Person und Profession sowie auf die Schulleitungen als ihre Kernzielgruppe (vgl. Huber, Arnz \& Klieme 2020). Denn zum einen sind Vertreter*innen der Schulaufsicht pädagogische Führungskräfte, die ihre eigene Weiterentwicklung im Sinne einer stetigen Professionalisierung im Blick haben, zum anderen obliegt es der Schulaufsicht, die Führungskräfteentwicklung in der Einzelschule, d.h. der Schulleiter*innen und weiterer Mitglieder der Schulleitung, aktiv zu initiieren, $\mathrm{zu}$ begleiten, wie auch sie $\mathrm{zu}$ beraten und $\mathrm{zu}$ unterstützen, wenn es darum geht, weitere Personen aus dem Kollegium für schulische Führungsaufgaben zu gewinnen und entsprechend zu fördern. Diese Aufgaben hat sie nicht nur der Schule, sondern auch ihrem eigenen Nachwuchs gegenüber. In beiden Fällen bedeutet Personalgewinnung (frühzeitige) Personalentwicklung auf Schulebene.

Strategische Überlegungen dieser Art sind umso dringender, als diese zentralen Stellen bereits seit Jahren oft nicht besetzt ist (vgl. Huber, 2008). Es zeichnet sich $a b$, dass derzeit und zukünftig umfangreiche Stellenbesetzungsverfahren erforderlich sind und somit eine entsprechend frühzeitig einsetzende und langfristig angelegte Nachwuchsrekrutierung und -förderung notwendig sind. Der gegenwärtige Mangel an Lehrkräften verschärft diese Problematik und wirft die Frage auf, ob nicht auch für die Gruppe der Schulaufsicht intensive Personalgewinnung betrieben werden sollte, zum Beispiel durch die Schaffung eines Pools an potenziellen Führungskräften für alle Ebenen des Schulsystems. Auf regionaler Ebene gibt es z. B. in Thüringen seit 2009 einen Nachwuchsführungskräftepool (vgl. Althaus, 2013; siehe Thüringer Schulportal, o.D.). Solche Maßnahmen der gezielten und frühzeitigen „Bewerberansprache“ und „Personalwerbung“ sind Kennzeichen eines effektiven und effizienten Personalmarketings.

Ein weiterer wichtiger Schritt, der im damaligen Kooperationsprojekt noch unberücksichtigt blieb, ist die Entwicklung geeigneter Instrumente zur Gewinnung, Auswahl, 
Vorbereitung und Einführung interessierter Kolleg*innen für die Schulaufsicht sowie Überlegungen zur Gestaltung der Arbeitsbedingungen. Genauso wie sich die Erkenntnis durchgesetzt hat, dass Schulleitung ein eigenes Berufsbild darstellt, das Schnittmengen, aber auch viele Unterschiede zur Lehrer*innentätigkeit aufweist und auch andere Kompetenzen erfordert, muss klar werden, dass natürlich auch Schulaufsicht nicht einfach nur eine Verlängerung von Schulfunktionslaufbahnen darstellt, sondern ebenfalls als ein eigenes Berufsbild mit spezifischen Kompetenzanforderungen zu verstehen ist.

\section{Literatur und Internetquellen}

Althaus, B. U. (2013). Nachwuchsführungskräftepool. In S. G. Huber (Hrsg.), Handbuch Führungskräfteentwicklung. Grundlagen und Handreichungen zur Qualifizierung und Personalentwicklung im Schulsystem (S. 202-206). Köln: Wolters Kluwer Deutschland.

Arnold, R. (2010). Schulleitung als Personalentwickler. In H.-G. Rolff (Hrsg.), Führung, Steuerung, Management (S. 81-98). Seelze: Friedrich.

Buchen, H., Burkhard, C. (2002). Schule und Schulaufsicht lernen gemeinsam. In H.-G. Rolff \& H.-J. Schmidt (Hrsg.), Brennpunkt Schulleitung und Schulaufsicht. Konzepte und Anregungen für die Praxis (S. 43-59). Neuwied, Kriftel: Luchterhand.

Drahmann, M., \& Huber, S. G. (2017). Aktuelle Entwicklungen und Herausforderungen in der Lehrerbildung. In M. Drahmann \& S. G. Huber (Hrsg.), SchulVerwaltung spezial. Zeitschrift für Schulgestaltung und Schulentwicklung, 19 (5), 196-199.

Frey, A., \& Jung, C. (2011). Kompetenzmodelle und Standards in Lehrerbildung und Lehrerberuf. In E. Terhart, H. Bennewitz \& M. Rothland (Hrsg.), Handbuch der Forschung zum Lehrerberuf (S. 540-572). Münster: Waxmann.

Huber, S. G. (2003). Qualifizierung von Schulleiterinnen und Schulleitern im internationalen Vergleich: Eine Untersuchung in 15 Ländern zur Professionalisierung von pädagogischen Führungskräften für Schulen (Wissen \& Praxis Bildungsmanagement). Kronach: Wolters Kluwer.

Huber, S. G. (2008). Expertise „Führungskräfteentwicklung im Schulwesen. Professionalisierungsbedarf von Schulleitungen infolge zunehmender Eigenverantwortung von Schulen" für die Bertelsmann Stiftung. Gütersloh.

Huber, S. G. (2013). Handbuch Führungskräfteentwicklung. Grundlagen und Handreichungen zur Qualifizierung und Personalentwicklung im Schulsystem. Köln: Carl Link \& Wolters Kluwer Deutschland.

Huber, S. G. (2019). Entwicklung von Kompetenzen für pädagogische Führung: Ein Kontinuum über die gesamte Lehrerbildung. In W. Böttcher, U. Heinemann, C. Kubina \& B. Priebe (Hrsg.), Steuerung und Qualitätsentwicklung im Fortbildungssystem. Probleme und Befunde - Standardbildung und Lösungsansätze (S. 152-181). Seelze: Kallmeyer \& Klett.

Huber, S. G. (2020a). Rolle und Aufgaben von Schulaufsicht in der Schulentwicklung. In S. G. Huber, S. Arnz \& T. Klieme (Hrsg.), Schulaufsicht im Wandel. Rollen und Aufgaben neu denken (S. 121-137). Stuttgart: Raabe.

Huber, S. G. (2020b). Germany: Education State Agencies in Germany - Their Organization, Role and Function in School Governing and Quality Management. In H. Ärlestig \& O. Johansson (Hrsg.), Educational Authorities and the Schools. Organisation and Impact in 20 States (S. 169-187). Dordrecht: Springer. https://doi.org/10.1007/978-3-030-38759-4 
Durchgängige und systematische schulische Führungskräftegewinnung und -entwicklung |

Huber, S. G., Arnz, S., \& Klieme, T. (Hrsg.). (2020). Schulaufsicht im Wandel. Rollen und Aufgaben neu denken. Stuttgart: Raabe.

Huber, S. G., Schneider, J. A., \& Pruitt, J. (2020). Schulen mit besonderen Herausforderungen - Besonders belastete Schulen. SchulVerwaltung spezial, 4.

Huber, S. G., \& Hiltmann, M. (2011). Competence Profile School Management (CPSM) - an Inventory for the Self-Assessment of School Leadership. Educational Assessment, Evaluation and Accountability, 23 (1), 65-88. https://doi.org/10.1007/s11092-010-9111-1

Huber, S. G., Schneider, N., Gleibs, H. E., \& Schwander, M. (2013). Leadership in der Lehrerbildung. Entwicklung von Kompetenzen für pädagogische Führung. Herausgegeben von der Stiftung der Deutschen Wirtschaft (sdw) und der Robert Bosch Stiftung (RBS).

Huber, S. G., Stiftung der Deutschen Wirtschaft (sdw) \& Robert Bosch Stiftung (RBS) (Hrsg.). (2015). Schule gemeinsam gestalten. Entwicklung von Kompetenzen für pädagogische Führung. Beiträge zu Leadership in der Lehrerbildung. Münster: Waxmann.

Huber, S. G., Tulowitzki, P., \& Hameyer, U. (2017). Curriculum and School Leadership: Adjusting School Leadership to Curriculum. In M. Uljens \& R. M. Ylimaki (Hrsg.), Bridging Educational Leadership, Curriculum Theory and Didaktik. Non-affirmative Theory of Education (S. 309-332). Dordrecht: Springer. https://doi.org/10.1007/978-3319-58650-2_9

Liebel, H. J., \& Oechsler, W. A. (1994). Handbuch Human-Resource-Management. Wiesbaden: Gabler.

Maaz, K., Huber, S. G., Arnz, S., \& Klieme, T. (2020). Schulaufsicht gestern, heute und morgen: tradierte und neue Aufgaben und Rollen. In S. G. Huber, S. Arnz \& T. Klieme (Hrsg.), Schulaufsicht im Wandel. Rollen und Aufgaben neu denken (S. 341-366). Stuttgart: Raabe.

Missal, S. (2017). Professionalisierung der Schulaufsicht. In S. G. Huber \& N. Schneider (Hrsg.), SchulVerwaltung spezial. Zeitschrift für Schulgestaltung und Schulentwicklung, 19 (3), 128-131.

Projektgruppe „Qualitätsmanagement der Schulaufsicht“ der Senatsverwaltung für Bildung, Wissenschaft und Forschung (2007). Abschlussbericht. Berlin: Senatsverwaltung für Bildung, Wissenschaft und Forschung. Zugriff am 05.03.2021. Verfügbar unter https:// digital.zlb.de/viewer/resolver?urn=urn:nbn:de:kobv:109-opus-212652.

Rolff, H.-G. (1998). Schulaufsicht und Administration in Entwicklung. In H. Altrichter, W. Schley \& M. Schratz (Hrsg.), Handbuch der Schulentwicklung (S. 190-217). Innsbruck: Studien.

Rosenbusch, H. S. (1994). Lehrer und Schulräte. Ein strukturell gestörtes Verhältnis. Bad Heilbrunn: Klinkhardt.

Rosenbusch, H. S., \& Huber, S. G. (2018). Schulen als Orte organisationspädagogischer Forschung und Praxis. In M. Göhlich, A. Schröer \& S. M. Weber (Hrsg.), Handbuch Organisationspädagogik (S. 745-755). Wiesbaden: Springer. https://doi.org/10.1007/ 978-3-658-07512-5_69

Sassenscheidt, H. (2020). Personalentwicklung der Schulaufsicht: Impulse und Leitideen. In S. G. Huber, S. Arnz \& T. Klieme (Hrsg.), Schulaufsicht im Wandel. Rollen und Aufgaben neu denken (S. 243-269). Stuttgart: Raabe.

Schick, N. (2013). Nachwuchskräftegewinnung und -förderung. In S. G. Huber (Hrsg.), Handbuch Führungskräfteentwicklung. Grundlagen und Handreichungen zur Qualifizierung und Personalentwicklung im Schulsystem (S. 195-201). Köln: Wolters Kluwer Deutschland.

Schmelzer, F., \& Löffler, J. (2020). Anspruch und Wirklichkeit: Vom erschöpften Alleskönner zum wirksamen Supervisor der Schulentwicklung. In S. G. Huber, S. Arnz \& T. Klieme (Hrsg.), Schulaufsicht im Wandel. Rollen und Aufgaben neu denken (S. 88-108). Stuttgart: Raabe. 
Thüringer Schulportal (o. D.). Führungskräfte - Führungskraft werden. Zugriff am 05.03.2021. Verfügbar unter: https://www.schulportal-thueringen.de/fuehrungskraefte/fuehrungs kraft_werden.

Trageser, H., \& Huber, S. G. (2020). Schulen in kritischer Lage: Wie kann und sollte Schulaufsicht unterstützen? In S. G. Huber, S. Arnz \& T. Klieme (Hrsg.), Schulaufsicht im Wandel. Rollen und Aufgaben neu denken (S. 209-234). Stuttgart: Raabe.

Stephan Gerhard Huber, Prof. Dr., geb. 1971, Leiter des Instituts für Bildungsmanagement und Bildungsökonomie (IBB) an der Pädagogischen Hochschule Zug.

E-Mail: stephan.huber@phzg.ch

Nadine Schneider, M. A., geb. 1979, Wissenschaftliche Mitarbeiterin in der Arbeitsgruppe "Bildungsmanagement" der Erfurt School of Education (ESE) an der Universität Erfurt und freie Projektmitarbeiterin am Institut für Bildungsmanagement und Bildungsökonomie der Pädagogischen Hochschule Zug

E-Mail: nadine.schneider@phzg.ch

Korrespondenzadresse: Pädagogische Hochschule Zug, Zugerbergstr. 3, CH-6300 Zug 
Christiane Montandon \& Marie Brégeon

\title{
Rekrutierung, Qualifizierung, Status und Karriereoptionen von Schulleiter ${ }^{*}$ innen staatlicher Sekundarschulen in Frankreich ${ }^{1}$
}

\section{Zusammenfassung}

Gegenstand des Berichts sind die aktuell in Frankreich geltenden Verfahren für die Rekrutierung und Qualifizierung von Schulleiter*innen der Sekundarschulformen collège und lycée. Dargestellt werden das Zulassungs- und Auswahlverfahren (concours) für die spezielle Qualifizierung, der Qualifizierungsverlauf und verschiedene Möglichkeiten für die weitere Karriereplanung und Profilentwicklung. Ziel des Berichts ist es, verständlich zu machen, weshalb Lehrkräfte wie auch Personen aus bestimmten anderen Berufsfeldern sich für eine Schulleitungsstelle interessieren und qualifizieren und welche weiteren Optionen sie damit verbinden.

Schlüsselwörter: Schulleitungsqualifizierung Frankreich, Qualifizierungsverlauf, Einstellungsvoraussetzungen, Aufgabenbereich, Karrierewege

\section{Recruitment, Qualification, Status and Career Options of Headteachers for the Secondary School Forms in France}

\begin{abstract}
The article deals with current recruitment and qualification procedures of headteachers for the secondary school forms collège and lycée in France. The admission and selection procedure (concours) for the special qualification, the qualification process, and different possibilities for further career planning and profile development are presented. The aim of the report is to clarify why teachers as well as people from certain other professional fields are interested in and choose to qualify for a school leadership position and which further options they associate with it.
\end{abstract}

Keywords: headteacher qualification, France, qualification process, recruitment requirements, scope of duties, career paths

1 Bei diesem Beitrag handelt es sich um einen Originalbeitrag, der von den Autorinnen in französischer Sprache verfasst und von Marianne Krüger-Potratz für die Veröffentlichung in der DDS ins Deutsche übersetzt wurde. Der Titel des französischen Originalbeitrags lautet „Recrutement et formation des chefs d'établissement: quels statuts pour les cadres du personnel de l'Éducation Nationale dans le secondaire en France?" 
Anders als in Deutschland ist es in Frankreich nicht möglich, die Qualifizierungsmodalitäten wie auch das Tätigkeitsfeld von Schulleiter ${ }^{\star}$ innen von Grund- und Sekundarschulen so darzustellen, als handele es sich um eine Berufsgruppe. Die Unterschiede hinsichtlich des Aufgabenbereichs und des Status von Primarschulleiter*innen (directeurs, directrices d'école primaire) und Sekundarschulleiter ${ }^{\star}$ innen (chefs détablissement d'enseignement secondaire - principal, proviseur) sind zu bedeutend, ${ }^{2}$ auch wenn es Änderungen in der Qualifizierung der Lehrkräfte gegeben hat, um die beiden Ausbildungswege einander anzugleichen. Kürzlich wurde sogar vorgeschlagen, den Schulleiter*innen von Primarschulen mehr Entscheidungsmacht zuzugestehen; allerdings wurde dies seitens der Vertreter*innen des Berufsstandes abgelehnt. ${ }^{3}$ Der Statusunterschied zwischen denen, die eine Grundschule, und denen, die eine Schulform der Sekundarstufe 1 (collège) oder der Sekundarstufe 2 (lycée) leiten, lässt erkennen, wie schwierig die Angleichung und Verknüpfung dieser beiden Schulleitungssysteme in Frankreich ist. ${ }^{4}$

Für Schulleiter*innen an Grundschulen hat sich bisher wenig verändert. Der Aufgabenbereich der Führungskräfte an Sekundarschulen hingegen ist seit ungefähr zehn Jahren kontinuierlich verändert und erweitert worden. Waren sie schon immer die Vertreter ${ }^{\star}$ innen des Staates in der Schule und somit vor allem anderen für Rechts- und Verwaltungsangelegenheiten zuständig, so sind sie heute auch Teammanager*innen, herausgefordert durch ständige Reformen und neue Technologien als pädagogische Arbeitsmittel wie auch als Steuerungsinstrumente (Progin, Etienne \& Pelletier, 2019; Barrère, 2006). Neu ist, dass sie zusätzlich zu ihren Verwaltungsaufgaben einen „pädagogischen Auftrag“ erhalten haben: die innerschulische Gestaltung und die Förderung und Weiterentwicklung der Zusammenarbeit der Lehrkräfte und des schulischen Personals zur Umsetzung der verschiedenen Reformvorhaben. Um die damit verbundene Vielzahl von Interventionsmöglichkeiten wahrnehmen und koordinieren zu können, müssen sie verschiedene Kompetenzen erwerben:

2 Grundschuldirektor*innen werden - im Unterschied zu den Schulleiter*innen von Sekundarschulen - ausschließlich aus der Lehrer*innenschaft rekrutiert. Sie sind für innerschulische Verwaltungs- und Organisationsaufgaben zuständig; sie sind nicht Vorgesetzte der Kolleg*innen (anschaulich hierzu Zickgraf, 2006). Im Kontext der von der Macron-Regierung eingeleiteten Schulreform soll der Status von Primarschulleiter ${ }^{*}$ innen dem der Leiter ${ }^{\star}$ innen von Sekundarschulen angeglichen werden (MENJS / Gesetz, 2019). (Anm. d. Übers.)

3 Für die vom Bildungsministerium im November 2019 durchgeführte Befragung von Grundschuldirektoren siehe MENJS / Befragung, 2019. (Anm. d. Übers.)

4 Anmerkung zum Schulsystem: Nach dem „Gesetz für eine Schule des Vertrauens“ von 2019 (Loi pour une école de confiance; MENJS / Gesetz, 2019) beginnt die Schulpflicht mit dem dritten und endet mit dem 18. Lebensjahr. Bis zum Ende der Sekundarstufe I trennen sich die Bildungswege der Kinder und Jugendlichen nicht: drei Jahre Vorschule (école maternelle), fünf Jahre Grundschule (école primaire) und vier Jahre Sekundarschule (collège). Danach besteht die Möglichkeit, entweder drei weitere Jahre ein lycée zu besuchen (es gibt verschiedene Fachrichtungen), oder eine Ausbildung zu machen. - Auch die Lehrkräftebildung und -tätigkeit in Frankreich unterscheidet sich deutlich von der in Deutschland; siehe Campus Frankreich - Deutschland, o. D.; Villinger, 2018. (Anm. d. Übers.) 
Kompetenzen in Fragen des Personalmanagements, der Steuerung innovativer Projekte, des Auf- wie Ausbaus von Partnerschaften mit Einrichtungen und Personen des lokalen politischen, wirtschaftlichen und gesellschaftlichen Umfelds und die Kompetenz, diese Interventionsansätze zu koordinieren (siehe IH2EF, 27.06.2020).

Im Folgenden geht es daher um die Berufsgruppe der Sekundarschulleiter*innen, um die Voraussetzungen für deren Gewinnung und das staatliche, wettbewerbsförmig organisierte Zulassungs- und Auswahlverfahren (concours ${ }^{5}$ ) als Voraussetzung für die Teilnahme an der obligatorischen zentral angebotenen staatlichen Qualifizierungsmaßnahme, um den Verlauf der Qualifizierung und schließlich um die Faktoren, die für die weitere Karriereplanung und Profilentwicklung eine Rolle spielen. Ziel des Berichts ist es, die aktuellen Formen der Gewinnung und Qualifizierung schulischer Führungskräfte darzustellen und - unter Einbezug von zwei Interviews mit Schulleitern - verständlich zu machen, aus welchen Motiven sich Personen, insbesondere Lehrkräfte, für eine Führungsposition in einer Schule interessieren, sich für das entsprechende Qualifizierungsangebot bewerben und bei Erfolg, infolge des Statuswechsels, dafür ihre Lehrtätigkeit aufgeben (siehe MENJS / Personal, 2020).

\section{Die Qualifizierung zu Schulleiter*innen für Sekundarschulen in Frankreich}

Seit 2018 ist die "Staatliche Hochschule für Bildung und Ausbildung" (Institut des Hautes Études de l'Éducation et de la Formation - IH2EF) mit Sitz in Poitiers landesweit für die einjährige Qualifizierung der Schulleitungs-Anwärter*innen zuständig. ${ }^{6}$ Die Qualifizierungsmaßnahme am IH2EF beginnt nach erfolgreichem Bestehen der schriftlichen und mündlichen Abschlussprüfungen des landesweiten concours. Die Zugelassenen werden einer Schule - einem collège oder einem lycée - als stellvertretende Schulleiter*innen zugewiesen; ihr Status ist der von „Auszubildenden“" (statut de stagiaire). Die „Auszubildenden“ können ihrerseits Wünsche bezüglich ihrer „Einsatzschule“ äußern, sowohl was die Schulform und den Schultyp als auch was die Lage der Schule angeht. Inwieweit ihren Wünschen entsprochen wird, hängt jedoch davon ab, wie sie beim concours abgeschnitten haben und dementsprechend auf der Rangliste platziert sind.

5 Die jährlich stattfindenden concours sind ein wichtiges Steuerungsinstrument für die Stellenbesetzung im öffentlichen Dienst in Frankreich. Zu den concours werden deutlich mehr Personen zugelassen, als für die offenen Stellen benötigt werden. Daher ist es möglich und auch üblich, sich mehrfach am concours zu beteiligen, um das gewünschte Ziel zu erreichen. (Anm. d. Übers.)

6 Für weitere Informationen siehe die Webseite des IH2EF, o. D. (Anm. d. Übers.) 
$\mathrm{Zu}$ den Aufgaben des Instituts gehört es, zusammen mit der jeweiligen académie ${ }^{7}$, d.h. dem jeweiligen Bildungsverwaltungsbezirk, die Inhalte und Modalitäten der Qualifizierung, die Zusammensetzung der Gruppen und die Praktika zu konzipieren, die Konzepte umzusetzen, die Qualifizierungsprozesse zu steuern und in dieser Weise die Anwärter*innen auf dem Weg zu ihrem neuen Verantwortungsbereich zu begleiten. Die Dauer der Qualifizierungsmaßnahme wie auch die Modalitäten derselben sind erst kürzlich mit Erlass vom 27.03.2020 geändert worden (MENJS / Erlass, 2020; siehe auch MENJS / Personal, 2020).

Die vom IH2EF und der académie organisierten Ausbildungseinheiten müssen insgesamt mindestens 154 Stunden umfassen; bei Bedarf können sie durch zusätzliche Ausbildungsmodule ergänzt werden. Dabei hat das rectorat der académie - gemäß Artikel 3 des Erlasses vom 27. März 2020 - darauf zu achten, dass das Qualifizierungsprogramm „personalisiert“ wird, d.h., dass in früheren Funktionen und Arbeitsbereichen erworbene Kenntnisse und Fähigkeiten bei der Planung des Qualifizierungsprogramms für die einzelnen Teilnehmenden berücksichtigt werden. Das Programm umfasst sowohl Module mit Präsenzveranstaltungen als auch ergänzende, zumeist digitale Fernstudienangebote. Die vom IH2EF - in Kooperation mit der académie und der jeweiligen „Einsatzschule“ - ausgearbeiteten Inhalte sind aufeinander abgestimmt.

Im Anschluss an diese einjährige Qualifizierung verbleiben die Teilnehmenden noch zwei Jahre als stellvertretende Schulleiter*innen an der Schule und nehmen an weiteren Fortbildungsveranstaltungen teil. Sie können in ihrer Ausbildungszeit ein Erkundungs-Praktikum (stage d'ouverture), z. B. in einem Unternehmen oder in einer (pädagogisch relevanten) Einrichtung außerhalb des Zuständigkeitsbereichs des Bildungsministeriums, ableisten oder an einer internationalen Mobilitätsaktion im Umfang von mindestens 30 Stunden teilnehmen, gegebenenfalls auf mehrere Zeiteinheiten aufgeteilt.

In Anlehnung an Universitätsmodule sind fünf Qualifizierungseinheiten entwickelt worden: (1) Schulische Organisationsgestaltung und Steuerung; (2) Leistungsfähigkeit des Bildungssystems; (3) Recht und staatliche Bildungspolitik; (4) Lernen und Erfolg; (5) Vernetzung von Akteuren in pluralen Räumen. Mit dem Ziel der Internationalisierung werden $\mathrm{zu}$ den Veranstaltungen auch ausländische Schulleiter ${ }^{\star}$ innen eingeladen, und die französischen Teilnehmer ${ }^{\star}$ innen können - bezogen auf ihren Aufgabenbereich - Praktika in anderen europäischen Ländern absolvieren.

7 Académie ist die Bezeichnung für einen der 30 Bildungsverwaltungsdistrikte, zusammengefasst in 18 Bildungsverwaltungsregionen. Das oberste Verwaltungsgremium ist das Rektorat (rectorat), dem der oberste Verwaltungsbeamte - der bzw. die Rektor ${ }^{\star}$ in (recteur / rectrice) - vorsitzt. Da diese Bezeichnungen im Deutschen auf andere Institutionen, Ämter und Positionen verweisen, werden in diesem Fall die französischen Institutions- und Amtsbezeichnungen im Text beibehalten. (Anm. d. Übers.) 
Die Ausbildung umfasst mehrere Abschnitte, die teils am IH2EF, teils von der académie, in der die Schule liegt, wie auch von der "Einsatzschule“ angeboten werden; in letzterer werden sie von einem bzw. einer Mentor ${ }^{\star}$ in und der Schulleitung unterstützt. Hinzu kommen Unterrichtseinheiten per Fernlehre auf der Online-Plattform

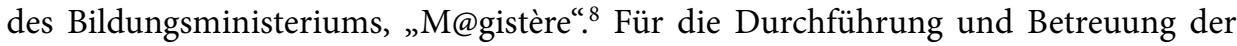
theoretischen wie der praktischen Ausbildungsanteile steht ein Netzwerk von 1.500 Lehrbeauftragten, darunter 400 assoziierte Mentorinnen, bereit.

Mit der Kombination von Theorie und Praxis in der Qualifizierung wird ein zweifaches Ziel verfolgt: die Einarbeitung in die neue Tätigkeit und Position wie auch der Erwerb der dafür benötigten, neuen beruflichen Kenntnisse und Kompetenzen. Zur Unterstützung des Sich-Einarbeitens in das neue Tätigkeitsfeld und der gesetzlich vorgeschriebenen „Personalisierung“ der Qualifizierung ist die digitale Plattform „Socrate“ entwickelt worden. Gleich nach der Zulassung zur Qualifizierungsmaßnahme stellen die "Auszubildenden“ Informationen über die von ihnen im Verlauf ihrer bisherigen beruflichen Karriere erworbenen Kompetenzen ein und besprechen mit ihrem bzw. ihrer Mentor ${ }^{\star}$ in, welche Ziele sie sich im Rahmen der Qualifizierungsmaßnahme und für den weiteren Verlauf ihrer beruflichen Karriere setzen möchten.

Da das IH2EF eine Unterabteilung der Personalabteilung des Bildungs- und des Wissenschaftsministeriums ist (IH2EF, 27.06.2020), wird die von ihm angebotene Qualifizierung für Führungskräfte nicht mit einem Universitätsdiplom abgeschlossen. Um dennoch denjenigen, die einen universitären Abschluss anstreben, die Möglichkeit zu bieten, anstelle der weiteren Schritte auf der Schulleitungslaufbahn ein Master2-Studium ${ }^{9}$ aufzunehmen, wurden mit verschiedenen Universitäten Abkommen über den Zugang zu bestimmten Master-2-Studiengängen geschlossen, z.B. zu den Studiengängen „Schulmanagement und Organisationsentwicklung“ (Management des organisations scolaires - M@dos), „Leitung und Steuerung schulischer Organisation“ (Gestion des organisations scolaires - Gedos) oder zu dem von der Universität Poitiers eingerichteten Masterstudiengang „Bildungstechnologie, Mediation, digitale Bildung“ (Ingéniérie, Mediation, e-Éducation - IME). Die Entscheidung für ein einjähriges universitäres Aufbaustudium anstelle der Fortsetzung der Schulleitungsweiterbildung ist zwar eine persönlich und freiwillig getroffene Entscheidung. Aber es drängt sich die Frage auf, mit welcher Einstellung diejenigen, die diese Entscheidung treffen, die Qualifizierung für Schulleiter*innen aufgenommen haben, ob sie nun gesetzlich vorgeschrieben ist oder nicht.

8 Die Onlineplattform „M@gistère“ wird für die Fort- und Weiterbildung aller im Bildungswesen Tätigen genutzt, darunter auch für die Qualifizierung zu Schulleiterinnen. (Anm. d. Übers.)

9 Der Master 2 ist ein einjähriges Aufbaustudium mit einer schriftlichen Abschlussprüfung; Ziel ist eine praxisorientierte Vertiefung bisheriger Studien. 


\section{Die Entwicklung von Karriereprofilen}

Seit 2001 sind Schulleiter*innen per Gesetz verpflichtet, nach einem Zeitraum von drei bis sieben Jahren an der Spitze derselben Institution ihre Versetzung zu beantragen. ${ }^{10}$ Diese Regelung bietet ihnen zugleich die Möglichkeit, ihre Karriere eigeninitiativ zu gestalten, indem sie sich auf eine aus ihrer Sicht interessante wie lukrative Stelle bewerben. Ein erster Schritt in diese Richtung ist die Möglichkeit, sich nach den drei Jahren Tätigkeit, Qualifizierung und Fortbildung als stellvertretende Schulleitung auf eine Schulleitungsstelle zu bewerben.

Für die Gestaltung der eigenen Karriere kommen unterschiedliche Überlegungen ins Spiel: der Wunsch, eine anspruchsvollere Aufgabe wahrzunehmen und/oder eine bessere Besoldung $\mathrm{zu}$ erreichen, die Aussicht auf interessante anschließende Karriereoptionen wie auch die Zusammenarbeit mit interessanten Partnern. Eine wichtige Grundlage für derartige Überlegungen ist die im Amtsblatt (Bulletin officiel) veröffentlichte aktuelle Fassung der Tabelle, aus der hervorgeht, welcher der vier Kategorien die einzelnen Schulformen und -typen gemäß Artikel 24 des Dekrets vom 11.12.2001 (MENJS / Dekret, 2001) zugeordnet sind. Die Zuordnung erfolgt nach quantitativen und qualitativen Merkmalen. Je nach Kategorie ändert sich mit dem Arbeits- und Aufgabenfeld auch die Höhe der Zulage. Eine erste Einstufung der Schulen und Schulformen erfolgt nach quantitativen Kriterien, d.h. der Zahl der Schüler*innen, wie Tabelle 1 zeigt:

Tab. 1: Beispiel für die Kategorisierung der Schulen nach der Zahl der Schüler*innen in 2020 - ein Indikator für die zu erwartende Gehaltszulage.

\begin{tabular}{|c|c|c|c|c|c|}
\hline \multicolumn{2}{|c|}{$\begin{array}{c}\text { Lycées } \\
\text { (allgemeinbildend) }\end{array}$} & \multicolumn{2}{|c|}{$\begin{array}{c}\text { Lycées } \\
\text { (berufsbildend) }\end{array}$} & \multicolumn{2}{|c|}{ Collèges } \\
\hline päd. Personal & Kategorie & Schüler*innen & Kategorie & Schüler*innen & Kategorie \\
\hline & 1 & bis zu 400 & 1 & bis zu 400 & 1 \\
\hline bis zu 750 & 2 & 400 bis 599 & 2 & 400 bis 699 & 2 \\
\hline 750 bis 999 & 3 & 600 bis 799 & 3 & 700 bis 999 & 3 \\
\hline $\begin{array}{l}1000 \text { und } \\
\text { mehr }\end{array}$ & $\begin{array}{c}4 \text { und } 4+ \\
\text { Sonder- } \\
\text { kategorie }\end{array}$ & 800 und mehr & 4 & 1000 und mehr & 4 \\
\hline
\end{tabular}

Quelle: eigene Darstellung

Die weitere Einstufung erfolgt nach qualitativen Kriterien durch die für Personalressourcen zuständige Abteilung des Ministeriums (Direction Générale des Ressources Humaines - DGRH). Höher eingestuft werden z.B. Schulen, die einem Regionalen Netzwerk von Schulen in sozialräumlich (besonders) deprivierter Lage angehören

10 Ausnahmen sind möglich, z.B. wenn die Pensionierung kurz bevorsteht. 
(Réseau d'Éducation Prioritaire - REP / REP+) sowie Förderschulen, Schulen mit beruflicher Ausbildung, Einrichtungen, die eine Ausbildung für Wissenschafts-, Technologie- und Gesundheitsberufe anbieten, verschiedene regionale bzw. lokale Verbünde von Schulen und Bildungseinrichtungen für Erwachsene oder auch ein lycée mit Vorbereitungsklassen für die Grandes Écoles. Da die Versetzung in eine höherstufige Bildungseinrichtung stufenweise erfolgt, bietet es sich an, den beruflichen Aufstieg strategisch zu planen. ${ }^{11}$

Bei allen strategischen Überlegungen spielt auch die schon angesprochene Erweiterung des Aufgabenbereichs der Schulleitungen, die Aufgabe der „pädagogischen Führung“ im Kontext der vom Bildungsministerium geforderten Verknüpfung von administrativen und pädagogischen Schulleitungsfunktionen, eine Rolle. Die Schulleiter*innen sind seitdem Mitglieder und Vorsitzende der „Bildungsgemeinschaft“ (communauté éducative), der das Verwaltungs- und pädagogische Personal, die Schüler*innen und deren Eltern sowie Vertreterinnen der für die Schule zuständigen lokalen Behörden und weitere institutionelle wie soziale Akteure angehören. Somit haben Schulleiter*innen auch Einfluss auf die Gestaltung der Lehr-Lern-Prozesse, um allen Schüler*innen zu ermöglichen, erfolgreich lernen können. ${ }^{12}$

Möglicherweise ist diese - für Frankreich - relativ neue Rollenverteilung einer der Faktoren, die Lehrkräfte, die schon alle Facetten ihrer Lehrer*innentätigkeit kennengelernt haben und befürchten, in Routine zu verfallen, dazu motiviert, sich für den Statuswechsel zu entscheiden: mehr Verantwortung zu übernehmen, sich komplexeren Herausforderungen $\mathrm{zu}$ stellen mit Aussicht auf eine Karriere, im Verlauf derer immer wieder viel Neues zu entdecken ist und viele Anregungen zu erhalten sind. Um von den Betreffenden selbst die verschiedenen Beweggründe zu erfahren, die Lehrkräfte ${ }^{13}$ dazu veranlassen, ihre Lehrer*innenstelle zugunsten einer Schulleitungskarriere aufzugeben, haben wir Interviews mit aktiven wie ehemaligen Schulleiter*innen von Sekundar-1-Schulen (principal de collège) und Sekundar2-Schulen (proviseur de lycée) geführt. Wir haben sie u.a. gefragt, was sie motiviert hat ${ }^{14}$ und ob sie im Verlauf ihrer Karriere, angesichts des mehrfachen Stellenwechsels, nicht nur das Gefühl haben, „Karriere zu machen“ und ihre berufliche Identität zu stärken, sondern ob auch die jeweils damit verbundenen materiellen Vorteile eine Rolle gespielt haben, sowohl die mögliche Gehaltsverbesserung als auch das mit dem Schulleitungsstatus in der Regel verbundene Recht auf eine Dienstwohnung, ein - vor allem in Großstädten - unschätzbarer Vorteil. Anzumerken bleibt, dass die

11 Anzumerken ist, dass der berufliche Aufstieg von Frauen immer noch geringer ausfällt als der von Männern.

12 Eines der wichtigen Themen in diesem Zusammenhang ist die hohe Zahl von Schulabbrecher*innen (siehe MENJS / Prävention, 2020). (Anm. d. Übers.)

13 Es sei daran erinnert, dass am concours und damit auch an der Qualifizierungsmaßnahme auch Personen teilnehmen können, die zuvor nicht im Bildungsbereich tätig waren; diesbezüglich notwendige Informationen sind der jeweiligen Ausschreibung zu entnehmen.

14 Zitate aus Interviews im Kontext einer noch laufenden Untersuchung. (Anm. d. Übers.) 
Besoldung der Schulleiter*innen große Unterschiede aufweist, je nach Schulform, dem fachlichen Profil der Schule, deren Größe und Lage bzw. nach Art des jeweiligen Schulverbunds. ${ }^{15}$

\section{Karrierewege aus Sicht von Schulleiter*innen - zwei Beispiele}

Einer der Gründe eines inzwischen aus dem aktiven Dienst ausgeschiedenen Schulleiters war, dass er sich nach zehn Jahren Unterrichtstätigkeit langweilte: ${ }^{16}$ "Ich hatte auf allen Klassenstufen unterrichtet, ich hatte alles durchlebt." Daher habe er nach Möglichkeiten gesucht, seine „berufliche Identität zu erweitern“ und seine „Kompetenzen weiter auszubauen“. Sein damaliger Schulleiter habe ihn auf die Idee gebracht habe, diesen Weg einzuschlagen, und ihn ermutigt. "Ich sehe Sie als Schulleiter; Sie haben alle dafür notwendigen Fähigkeiten!", habe er gesagt und dann folgende, für die Tätigkeit als Schulleiter erforderlichen Kompetenzen aufgezählt:

- sich im Umgang mit Menschen wohl zu fühlen, zuhören und sich einfühlen zu können;

- Entscheidungen treffen und diese in transparenter Weise gegenüber seinen Kolleg*innen, Mitarbeiter^innen und dem Kollegium vertreten zu können;

- auf Zusammenarbeit zu setzen, das Gespräch zu suchen, eigene Entscheidungen $\mathrm{zu}$ erklären, individuelle Initiativen zusammenzuführen, insbesondere dank der Anregungen des jeweiligen „Pädagogischen Rats"17, Projekte unter Einbeziehung der Lehrkräfte und zu deren Zufriedenheit umsetzen zu können, weil dies die Schule positiv verändert;

- zu wissen, wie man die Vorgaben der nationalen Bildungspolitik unter Wahrung eines gewissen Autonomiespielraums, d.h. mit einer gewissen Distanz zum System, umsetzt.

15 Unterschieden wird z.B. zwischen allgemein- oder berufsbildendem lycée, dem collège mit oder ohne Förderschulzweig, der Förderschule, der regionalen Sekundar-1-Schule mit Tagesinternatsschule für Kinder beruflich Reisender oder dem lycée mit Vorbereitungsklassen für die Grandes Écoles, d.h. für eine der fachlich spezialisierten Hochschulen, an denen das Führungspersonal für den Staatsdienst, die Wirtschaft und die Wissenschaft ausgebildet wird. Beispiele für lokale Schulverbünde sind der Zusammenschluss öffentlicher Einrichtungen für die Erwachsenenweiterbildung und Lehrlingsausbildung oder der Verbund von collège und lycée, die ihre personellen und materiellen Ressourcen zusammenlegen, um Weiterbildungsaktivitäten für Erwachsene und die Ausbildung von Lehrlingen zu organisieren.

16 Unabhängig davon, ob es in diesem Fall eine Rolle gespielt hat, ist festzuhalten, dass Lehrkräfte in Frankreich i.d.R. nur ein Fach unterrichten; zu weiteren Unterschieden in der Ausbildung siehe: Campus France Deutschland, o. D. (Anm. d. Übers.)

17 Der Conseil Pédagogique de l'école (CP) ist ein 2005 verbindlich eingeführtes Gremium, dessen Mitglieder - auf Vorschlag der in der Schule Tätigen - vom bzw. von der Schulleiter*in ernannt werden. Der bzw. die Schulleiter ${ }^{\star}$ in ist auch zugleich Vorsitzende ${ }^{\star} r$ des CP. Der CP ist zuständig für ein breites Spektrum pädagogischer Fragen. 
Dieser Zuspruch sei für seine Entscheidung, sich für die in Poitiers angebotene Ausbildung zu bewerben, ausschlaggebend gewesen. Alle von ihm im Interview genannten Gründe beziehen sich letztlich auf den Wunsch, seine beruflichen Kompetenzen zu erweitern. Welche Rolle die mit dem Schulleitungsposten ebenso verbundene finanzielle Verbesserung gespielt hat, war trotz Nachfrage nicht eindeutig zu klären.

Ein anderer Schulleiter nennt weitere Beweggründe: Er hatte seine 14-jährige Karriere als Pädagogischer Berater ${ }^{18}$ (Conseiller Principal d'Éducation - CPE) und stellvertretender Schulleiter (principal adjoint) eines collège begonnen. Anschließend hatte er im Zuge der Versetzungen nicht nur die Schule, sondern auch die Schulform gewechselt: Er war Schulleiter eines anderen collège (principal) und danach Schulleiter (proviseur) an verschiedenen lycées. Er sieht die Vorteile der gesetzlich in bestimmten Zeiträumen vorgesehenen Versetzungen und des damit verbundenen Wechsels des jeweils spezifischen Umfelds darin, dass es stets eine neue Berufswelt zu entdecken gebe: Je nach Größe und Lage der Schule (städtische oder ländliche Umgebung) - immer gelte es, mit unterschiedlichen Lehrkräfteteams zusammenzuarbeiten, immer sei das Arbeitsklima ein anderes, und die finanziellen Vorteile seien, je nach der erforderlichen Arbeitsbelastung, von Schultyp zu Schultyp sehr unterschiedlich. Der finanzielle Aspekt habe für seine jeweilige Wahl keine ausschlaggebende Rolle gespielt. Ihn habe vor allem die Gelegenheit gereizt, etwas zu verändern: „die Linien zu verschieben“, wie er es formuliert. So habe er 2015, damals als Schulleiter eines collège, an der Umsetzung des Reformprojekts „Interdisziplinärer Praktischer Unterricht" (Enseignements Pratiques Interdisciplinaires - EPI) mitgearbeitet und 2018 als Schulleiter eines lycée an der Reform des Abiturs (baccalauréat ${ }^{19}$ ). Für die mit diesen Reformprojekten verbundenen Veränderungen sei stets viel Überzeugungsarbeit bei den Lehrkräften zu leisten gewesen; z. B. hätten sich diese im Fall des lycée in einen Konkurrenzkampf der Fächer verstrickt, und auch die Eltern und Schüler*innen hätten Bedenken gehabt, die Modalitäten des Abiturs zu ändern. Im Fall der Reform der collèges und der vorgesehenen interdisziplinären Zusammenarbeit seien die Lehrkräfte geteilter Meinung gewesen; einige hätten sogar den Verlust ihrer fachlichen Identität befürchtet. Der Schulleiter hat die Gestaltung solcher Veränderungen als „intensiv, kompliziert und aufregend“ erlebt; er habe die Gelegenheiten nutzen können, um seine Führungsstrategien auszubauen - immer mit dem Bestreben, alle Kolleg*innen einzubeziehen.

18 Der Conseiller Principal d'Éducation (CPE) ist für das Schulleben und die „Entwicklung und Umsetzung der schuleigenen Bildungsstrategie“ einschließlich der Pflege außerschulischer Partnerschaften und für die Sicherstellung der bestmöglichen Lernbedingungen aller Schüler*innen zuständig. (Anm. d. Übers.)

19 Die Reform des Abiturs geht weiter; für die Änderungen der verschiedenen lycée-Typen siehe das Stichwort „baccalauréat“ auf der Seite des Bildungsministeriums: https://www.education.gouv.fr/. 
Dieser Schulleiter versteht die Auseinandersetzung mit der komplexen Aufgabe der Umgestaltung der Organisation des Unterrichtsangebots und des Schullebens als Herausforderung und Beleg dafür, dass er qua Amt über ein gewisses Maß an Entscheidungsmacht verfügt. Er sieht es als Herausforderung, dass er zwischen den bildungspolitischen Vorgaben und den Vor- und Einstellungen der Lehrer*innenteams immer wieder habe vermitteln und Lösungen finden müssen, und dies oftmals in Situationen, die aufgrund der hierarchischen Strukturen spannungsgeladen waren.

\section{Schulleiter*innenfortbildung - ein Problem?}

Die beiden zitierten Schulleiter betonten die Komplexität der Anforderungen im Umgang mit den institutionellen und strukturellen Gegebenheiten, den formalisierten Rollen und daraus resultierenden Beziehungsproblemen mit dem und im Kollegium. Beide wünschten sich daher, mehr Zeit für Fortbildung aufbringen zu können. Zugleich räumten sie ein, dass sie dafür eigentlich keine Zeit hätten. Schließlich müssten sie einerseits ihre Managementkompetenzen weiterentwickeln, da sich nach fünf oder sechs Jahren die Vorstellungen und Erwartungen sowohl der Lehrkräfte als auch des Ministeriums infolge neuer Richtlinien verändert hätten, vor allem aufgrund der inzwischen eingeführten teilweisen Autonomisierung der Schule. Andererseits gebe es auf nationaler Ebene keinen Raum und keinen Ort für die gemeinsame Analyse der in der Praxis auftretenden Schwierigkeiten und für einen Erfahrungsaustausch über den Umgang damit. Die Fortbildungspolitik, wie sie in dem von jeder académie aufgestellten und veröffentlichten Jahres-Aktionsplan (Plan d'actions de formation - PAF) festgelegt ist, sei vor allem auf die spezifischen Bedürfnisse der betreffenden Region ausgerichtet; regional übergreifende Themen fänden hingegen zu wenig Beachtung.

Der ehemalige Schulleiter beklagt ausdrücklich das Fehlen berufsbegleitender Fortbildung; dies sei „ein echtes Problem“. In wichtigen Fragen habe er nur Hilfe von Seiten seiner Gewerkschaft, der SNPDEN (Syndicat National des Personnels de Direction de l'Éducation Nationale), erhalten. Dort habe er sich mit anderen Schulleiter*innen über wichtige Themen und Erfahrungen austauschen können, über den Umgang mit Widerständen, Steuerungsproblemen, Spannungen innerhalb von Teams oder über das Problem der hierarchischen Strukturen und formalisierten Rollenbeziehungen. Denn was stets eine Rolle spiele, sei sein Status als Führungskraft; er sei derjenige, der zwischen den Lehrkräften und den übergeordneten Behörden, den Vertreter*innen der académie und dem Ministerium vermitteln müsse.

Der andere Schulleiter berichtete von einem „CAFÉ-CHEF“, einem von Schulinspektor*innen gegründeten und geleiteten Treffen. Ausgehend von den Problemen, die anlässlich von Inspektionen beobachtet wurden und über den Einzelfall hinaus relevant seien, seien bei diesen Treffen jeweils verschiedene Themen zur Diskussion 
gestellt worden. Solche Initiativen seien jedoch keineswegs üblich, sondern sie hingen davon ab, wie das rectorat der jeweiligen académie dazu stehe. Seiner Erinnerung nach habe es in seiner académie zwischen 2005 und 2007 dreimal pro Jahr eine solche Möglichkeit gegeben, sich über Strategien für eine erfolgreiche Umsetzung von bildungspolitisch geforderten Veränderungen auszutauschen und damit auch zum Aufbau und zur Konsolidierung einer gemeinsamen Berufskultur beizutragen. Doch das damalige rectorat habe diese Treffen schließlich eingestellt. Nach wie vor bestehe jedoch die Hoffnung, dass solche Orte und Gelegenheiten wieder eingerichtet würden, u. a. auch, um etwas über die Reaktionen auf die jeweiligen Reformanforderungen in den anderen académies zu erfahren. Letzteres sei insbesondere für diejenigen Schulleiter*innen wichtig, die mit ihrer Versetzung nicht nur die Schule, sondern auch die académie wechselten. Er wünschte sich, dass es möglich sei, ein Netzwerk von Führungskräften in unterschiedlichen Positionen zu bilden und sich zwei- oder dreimal im Jahr zum Austausch von Kenntnissen und Erfahrungen zu treffen.

\section{Abschließende Überlegungen}

Der Beruf des Schulleiters bzw. der Schulleiterin ist in Frankreich attraktiv, trotz der Komplexität des Aufgabenbereichs und der zur Bewältigung der vielfältigen Aufgaben benötigten Fähigkeiten. Jedes Jahr meldet sich eine relativ hohe Anzahl von Personen für das nationale Auswahl- und Zulassungsverfahren (concours) an, um die schriftliche und mündliche Prüfung abzulegen. Im Schnitt wird ein Drittel der erfolgreichen Kandidat ${ }^{*}$ innen zur Qualifizierungsmaßnahme zugelassen, und zwar diejenigen, die aufgrund ihrer Prüfungsergebnisse auf der Rangliste auf einem Platz stehen, bis zu dem Einstellungen erfolgen. Im Schnitt sind es jedes Jahr etwa 700 Schulleitungsposten, die auf diese Weise besetzt werden. ${ }^{20}$

Im Unterschied zu Deutschland bedeutet der Wechsel von Lehrkräften in eine Schulleitungskarriere in Frankreich, dass sie den Lehrer*innenberuf aufgeben und in einen neuen Status mit einer neuen Berufskultur eintreten. Eine wichtige Rolle für die „Einpassung“ in den neuen Status spielen die Schulleitungsgewerkschaften; die Mehrheit der Schulleiter*innen ist gewerkschaftlich organisiert: vor allem in der SNPDEN oder auch in der Gewerkschaft Indépendance et direction - ID-FO. Die Gewerkschaften spielen eine entscheidende Rolle bei der Vernetzung von Schulleiter ${ }^{\star}$ innengruppen. Sie tragen dazu bei, dass deren Erfahrungen und Ansichten als Akteure im institutionellen Dialog Gewicht haben.

20 Eine deutlich kleinere Zahl von Schulleiter*innen wird auf der Basis einer Eignungsliste (liste d'aptitude) und eine noch kleinere Zahl über Abordnung (auf Zeit) rekrutiert. (Anm. d. Übers.) 


\section{Literatur und Internetquellen ${ }^{21}$}

Barrère, A. (2006). Sociologie des chefs d'établissement. Les managers de la République. Paris: PUF.

${ }^{*}$ Campus France Deutschland. (o. D.). Lehramt. Zugriff am 06.01.2021. Verfügbar unter: https://www.allemagne.campusfrance.org/lehramt.

${ }^{*} \mathrm{IH} 2 \mathrm{EF}$ (Institut des Hautes Études de l'Éducation et de la Formation). (27.06.2020). La formation statutaire des personnels d'encadrement. Zugriff am 20.12.2020. Verfügbar unter: https://www.ih2ef.education.fr/fr/formations/publics-de-nos-formations/la-formationstatutaire-des-personnels-d-encadrement/.

${ }^{*}$ MENJS / Dekret (Ministère de l'Éducation Nationale, de la Jeunesse et des Sports). (2001). Décret $n^{\circ} 2001-1174$ du 11 décembre 2001 portant statut particulier du corps des personnels de direction détablissement d'enseignement ou de formation relevant du ministère de léducation nationale. Zugriff am 28.12.2020. Verfügbar unter: https://www.legifrance. gouv.fr/loda/id/LEGITEXT000005631808/2017-05-22/.

${ }^{*}$ MENJS / Befragung (Ministère de l'Éducation Nationale, de la Jeunesse et des Sports). (2019). Synthèse de la consultation des directrices et directeurs d'école. Zugriff am 29.12.2020. Verfügbar unter: https://www.education.gouv.fr/synthese-de-la-consultati on-des-directrices-et-directeurs-d-ecole-41597.

*MENJS / Gesetz (Ministère de l'Éducation Nationale, de la Jeunesse et des Sports). (2019). Loi n²019-791 du 26 juillet 2019 pour une école de la confiance. Journal Officiel, 19 (0174), Text 3. Verfügbar unter: https://www.legifrance.gouv.fr/loda/id/ JORFTEXT000038829065/.

${ }^{*}$ MENJS / Erlass (Ministère de l'Éducation Nationale, de la Jeunesse et des Sports). (2020). Arrêté du 27 mars 2020 relatif à la formation professionnelle statutaire des personnels de direction d'établissement d'enseignement ou de formation relevant du ministre de l'éducation nationale et à la formation d'adaptation à l'emploi des personnels détachés dans ce corps. Journal Officiel, 20 (0145), Text 27. Zugriff am 29.12.2020. Verfügbar unter: https://www.legifrance.gouv.fr/jorf/id/JORFTEXT000041991318/.

${ }^{\star}$ MENJS / Personal (Ministère de l'Éducation Nationale, de la Jeunesse et des Sports). (2020). Etre personnel de direction. Zugriff am 28.12.2020. Verfügbar unter: https:// www.education.gouv.fr/etre-personnel-de-direction-6830.

${ }^{\star}$ MENJS / Prävention (Ministère de l'Éducation Nationale, de la Jeunesse et des Sports). (2020). La Lutte contre le décrochage. Zugriff am 08.01.2021. Verfügbar unter: https:// www.education.gouv.fr/la-lutte-contre-le-decrochage-scolaire-7214.

Montandon, F., Wagner, B., \& Krüger-Potratz, M. (Hrsg.). (2021). Les chefs détablissement face à la mobilité institutionnelle en Allemagne et en France - enjeux stratégiques et paradoxes. Paris: Téraèdre (im Druck).22

Progin, L., Etienne, R., \& Pelletier, G. (Dir.). (2019). Diriger un établissement scolaire. Louvain-La-Neuve: De Boeck.

*Villinger, M. (2018). Bildungsreform in Frankreich. Schul- und Hochschulbereich. Zugriff am 30.03.2021. Verfügbar unter: https://www.fachportal-paedagogik.de/literatur/vollanzei ge.html?FId=1171983.

*Zickgraf, P. (2006). Ein Tag im Leben eines französischen Schulleiters. Zugriff am 28.12.2020. Verfügbar unter: https://www.ganztagsschulen.org/de/2983.php.

21 Die mit * gekennzeichneten Publikationen sind für die deutsche Fassung eingefügt worden. (Anm. d. Übers.)

22 Die deutsche Fassung erscheint ebenfalls 2021 im Waxmann Verlag. 
Marie Brégeon, Dr., geb. 1977, promoviert in Erziehungswissenschaft, seit 2019 chef de cabinet de la rectrice de l'académie de Poitiers; von 2016 bis 2019 am Institut des Hautes Études de l'Éducation et de la Formation mit Sitz in Poitiers für die landesweit angebotene Qualifizierung zukünftiger Schulleiter*innen zuständig.

E-Mail: marie.bregeon@yahoo.fr

Christiane Montandon, Prof. em., geb. 1945, emeritierte Professorin für Erziehungswissenschaft an der Université Paris-Est Créteil und Mitglied der Forschergruppe LIRTES - EA 7313 zu Fragen der Veränderung von Bildungs- und sozialen Praktiken. E-Mail: montandon@u-pec.fr 


\title{
DISKUSSION ZUM SCHWERPUNKTTHEMA
}

DDS - Die Deutsche Schule

113. Jahrgang 2021, Heft 2, S. 174-187

https://doi.org/10.31244/dds.2021.02.05

CC BY-NC-ND 4.0 Waxmann 2021

Katja Kansteiner, Pierre Tulowitzki, Michael Krüger \& Christoph Stamann

Führungskräfteentwicklung in der Lehramtsausbildung

Thesendiskussion zu curricularen Ergänzungen des

Lehramtsstudiums

\section{Zusammenfassung}

Im Beitrag diskutieren wir die Aufnahme des Themenfeldes Personalführung und Management in das Lehramtsstudium über die Verankerung in den Kompetenzstandards und begründen, warum sein Fehlen als Lücke in der nötigen wissenschaftlichen Vorbereitung auf die spätere Berufsausübung gelten kann. Das Themenfeld Personalführung und Management in die erste Phase der Lehrer*innenbildung mitaufzunehmen, heißt für uns zugleich, zur Führungskräfteentwicklung beizutragen. Vor diesem Hintergrund skizzieren wir auch, was für einen möglichen Aus- oder Umbau der Curricula der ersten Phase der Lehrer*innenbildung von bereits existierenden MA-Weiterbildungsstudiengängen im Bereich Personalführung und Management gelernt werden könnte.

Schlüsselwörter: Lehrer*innenbildung, Führungskräfteentwicklung, Professionalisierung

\section{Leadership Development in Teacher Education}

Discussion of Theses Regarding a Curricular Expansion of Teacher Education

\begin{abstract}
In this article, we discuss the inclusion of the issues of leadership and management in the teaching education programs by adding them to the competence standards and we explain why its lack prevents from a necessary scientific preparation for the later professional practice. For us, expanding the issues of leadership and management into the first phase of teacher education also means contributing to leadership development. Against this background, we clarify furthermore whether a possible expansion or modification of the curricula of the first phase of teacher education can derive ideas from existing Master programs in the field of further training on leadership and management. Keywords: teacher education, leadership development, professionalization
\end{abstract}




\section{$1 \quad$ Einleitung}

Führungskräfteentwicklung wurde in den letzten 25 Jahren angesichts erweiterter Selbständigkeit von Schulen im deutschsprachigen Raum intensiv wissenschaftlich und konzeptionell-praktisch verfolgt (Arnold, Bonsen, Huber, Schratz \& Rolff, 2010). Dabei erstrecken sich die Forschungs- und Entwicklungsperspektiven vom Aufgaben- und Kompetenzprofil der Schulleitung (z.B. Rolff, 2010; Huber, Wolfgramm \& Kilic, 2013) über Hürden und Strategien der Nachwuchsgewinnung (z.B. Hankock \& Müller, 2012; Kansteiner \& Klose, 2017) bis hin zu angemessenen Weiterbildungsformaten, -verfahren und inhaltlichen Ausrichtungen (z.B. Tulowitzki, Hinzen \& Roller, 2019). Eine gemeinsame Referenz ist das Verständnis einer Schulführung, die darauf ausgerichtet ist, dass die Einzelschule einschließlich ihrer Koordination mit dem System funktioniert, und die sich zugleich umfassend in der Mitarbeiter*innenführung engagiert und Qualitätsentwicklung sichert (z. B. Thom, Ritz \& Steiner, 2002; Schratz et al., 2015). Der jetzt schon eine Weile andauernde Mangel an Bewerber*innen für die Schulleitung (insbesondere im Primarbereich) bereitet zugleich Sorgen (OECD, 2008; Cramer, Gross Ophoff, Pietsch \& Tulowitzki, 2020).

Aufgrund u.a. systemsteuernder Aufgaben wird die Schulführung mitunter als eigenständiges Berufsfeld umrissen (Buchen \& Rolff, 2006). Die Bedeutung der Führungskraft als Change Agent für die Qualitätssicherung an der Einzelschule ist umfassend dargelegt (Fullan, 2007); gleichzeitig wird Schulführung mittlerweile auch als Aufgabe mehrerer Personen konzeptualisiert (Hallinger \& Heck, 2010) bzw. die Beteiligung von Lehrkräften mit führendem Rollenanteil als Teacher Leadership ausgewiesen (Strauss \& Anderegg, 2020). Seit geraumer Zeit regt überdies der Blick auf Leadership zu einem prozessbezogenen Verständnis in einer sonst vor allem personen- und funktionsbezogenen Debatte an (Schratz et al., 2015). Deutlich wird auch, dass der Führungsbegriff containerartig genutzt wird und sowohl für die Bezeichnung einer Person oder eines leitendenden Teams als auch für eine Aufgabe bis hin zur Bezeichnung konkreten Handelns genutzt wird.

Mit Fragen zur Führung wird auch die Gruppe der Lehrkräfte in Verbindung gebracht, meist jedoch unter einer pädagogisch-unterrichtsbezogenen Perspektive, z. B. über ihre erzieherische Funktion und die didaktische Dimension des Classroom Managements. In diesem Beitrag argumentieren wir allerdings, dass Lehrkräfte weitaus umfangreicher mit Fragen von Führung befasst sind, nämlich bis hinein in die Felder von Personalführung und Management.

Für das Spektrum der Aufgabe „Führung“ in der Schule liegen verschiedene wissenschaftliche Ausdifferenzierungen vor; so werden z.B. nach Bonsen (2009) die Organisation des Schulbetriebs, zielgerichtete Führung, Innovationsförderung, Förderung von Fort- und Weiterbildung, Management sozialer Beziehungen und die 
Partizipation in der Entscheidungsfindung als zentrale Aufgabenfelder definiert. Führungshandeln ist dabei als kontingentes, durch den spezifischen Kontext geprägtes Verhalten schulischen Personals zu verstehen, das auf die effektive Beeinflussung anderer Personen im System (vgl. Tulowitzki \& Pietsch, 2020) sowie auf Personalmanagement, Qualitätsmanagement und Organisation und Verwaltung abzielt (Huber \& Schwander, 2015). Wenn wir im Folgenden auf Führung als Personalführung und Management rekurrieren und sie mit Blick auf die Lehrkräfte diskutieren, dann vor dem Hintergrund all dieser Aufgaben und der entsprechenden Kompetenzen, die dafür nötig sind.

Erkenntnisse über Anforderungen an gelingende Führung in die Führungskräfteentwicklung $\mathrm{zu}$ integrieren, erfolgt bislang hauptsächlich im Rahmen der Phase der Berufstätigkeit und adressiert - etwa in Form der Anforderungsprofile (MKJS BW, 2016) - vor allem jene, die für sich bereits den Aufstieg in die Schulleitung sehen. ${ }^{1}$ Während einerseits also in der Praxis die Angebote der Führungskräfteentwicklung mit Themen wie Personalführung und Management nur an aufstiegsinteressierte Lehrkräfte herangetragen werden, werden andererseits in der Fachdebatte Lehrkräfte insgesamt als „führende Subjekte“ (Bonsen \& Berkemeyer, 2014, S. 924) angesehen. Sie kommen in diesem Verständnis neben ihren schüler*innenbezogenen Führungsaufgaben regelmäßig auch mit Fragen der Personalführung und des Managements in Berührung und werden nicht zuletzt generell als Schulentwickler*innen (KMK, 2004) eingeordnet, d.h., es ist eine Überschneidung mit den originären Aufgaben der Schulleitung zu konstatieren. Um dieser Rolle auch entsprechen zu können, benötigen allerdings selbst jene, die gar nicht mit dem Aufstieg in die Leitungsposition befasst sind, ein umfassendes Verständnis für die Schule als Organisation mit ihren spezifischen Führungs- und Managementfragen jenseits des Pädagogischen.

Nachfolgend zeigen wir mit einem kurzen Vergleich der Führungskräfteentwicklung in Deutschland und im US-amerikanischen Raum, dass in letzterem bereits erste Wege beschritten werden, schon Lehramtsstudierende mit diesen Fragen zu befassen.

\section{Personalführungs- und Managementkompetenz vor der Qualifizierung von Schulleiter*innen}

In Deutschland werden Schulleiter ${ }^{\star}$ innen an öffentlichen Schulen quasi vollständig aus dem Pool der Lehrkräfte heraus rekrutiert. Obwohl der Prozess je nach Bundesland etwas variiert, lässt sich vereinfachend festhalten, dass sich interessierte Lehrkräfte auf frei werdende Stellen bewerben können. Von einer bestehenden pä-

1 Das Anforderungsprofil des Landes Baden-Württemberg wird als „Grundlage [...] für gezielte Personalentwicklungsmaßnahmen“ sowie als „Referenzpapier [...] bei der Personalauswahl“ eingeordnet (MKJS BW, 2016, S. 3). 
dagogischen Qualifizierung potenzieller Schulleiter*innen kann aufgrund ihrer beruflichen Erfahrung wie auch aufgrund des Studiums ausgegangen werden. Für die Aufgaben in der Leitungsposition werden Kompetenzen gemeinhin erst in entsprechenden Fortbildungsprogrammen aufgebaut. Wenn diese Aufgaben allerdings nicht nur aufstiegsinteressierte Personen betreffen, wie wir es mit unserer These nahelegen, sondern alle Lehrkräfte darin kompetent sein sollten, ist eine Lücke in der Lehrer*innenbildung zu konstatieren, da die entsprechenden KMK-Standards die eben skizzierten Personalführungs- und Managementthemen in weiten Teilen nicht enthalten (KMK, 2004/i.d. F. 2019). Im Kompetenzbereich „Innovieren“, der am ehesten entsprechende Kompetenzvorgaben für Lehrkräfte vermuten lässt, werden Grundlagen zur Organisation von Strukturen und in ersten Ansätzen auch zur Repräsentation in Form von Verantwortungsbewusstsein vorgegeben (ebd., S. $13 \mathrm{f}$.). In den weiteren Ausführungen wird dann vor allem noch auf die Mitwirkung bei der Schulentwicklung durch das Erlangen von Kenntnissen zur Bildungsforschung sowie zur Selbst- und Fremdevaluation abgezielt (ebd.). Während die Fähigkeit zur Kooperation gefordert wird, fehlen Aspekte der Personalführung und des Managements weitgehend. Hierunter würden beispielsweise fallen:

- ein Verständnis des wechselseitigen Machtverhältnisses innerhalb der dyadischen und gruppenbezogenen (Kollegium) Führungssituation zur Leitung entwickeln, Mechanismen der Mikropolitik identifizieren und konstruktiv mitwirken zu können;

- Strukturbildung im Dienst von Managementaufgaben verstehen und mit beraten zu können;

- die Bereitschaft zur Beteiligung an der Visionsentwicklung und ihrer Umsetzung aufzubauen und sich kundig in Changemanagement-Prozesse einbringen zu können;

- Steuerungsstrategien zu kennen und die angemessene Rolle für eine gelingende Umsetzung darin einnehmen zu können;

- leitende Aufgaben in der Teamentwicklung und Teamkoordination und entgegen dem Autonomie-Paritäts-Muster ${ }^{2}$ exponierte und mit mehr Befugnissen ausgestattete Aufgaben übernehmen zu können.

2 Das Autonomie-Paritäts-Muster (übernommen nach dem autonomy parity pattern; Lortie, 1972) beschreibt die Erwartung von Lehrkräften, dass alle Lehrkräfte als gleich(wertig) zu betrachten sind und sich eine Einmischung in ihren Unterricht aufgrund der notwendigen Handlungsautonomie verbietet. Diese „berufstypische Einstellung und Verhaltensnorm“ (Rothland, Biederbeck, Grabosch \& Heiligtag, 2017, S. 195) gilt als weit verbreitete Haltung, um sich angesichts der strukturell gegebenen Unsicherheit über pädagogisch richtiges Handeln zu schützen. Sie wird allerdings auch als Hürde für eine qualitätsverbessernde Intervention von außen eingestuft (Heinrich \& Altrichter, 2008) und steht einer systematischen und auf das Lernen abzielenden Lehrkräftekooperation im Weg, da in dieser Kompetenzdifferenzen anzuerkennen und zu nutzen sind sowie in der gemeinsamen Beratung letztlich „Einmischung“ in die eigene Praxis geschieht (Kansteiner, 2019). 
Derzeit wird allerdings deutlich, dass Personalführung und Management in den Standards der Lehrer ${ }^{\star}$ innenbildung so gut wie keine Rolle spielen, und es kann davon ausgegangen werden, dass Lehrkräfte im Allgemeinen nicht in die systematische Auseinandersetzung damit kommen, es sei denn, sie sind aufstiegsinteressiert oder gar schon als Schulleiterin ernannt. Es sei am Rande erwähnt, dass auch die im bundesweiten Überblick verpflichtenden Qualifizierungsmaßnahmen zur Schulleitung in Deutschland (Tulowitzki, Hinzen \& Roller, 2019) different ausfallen: Sie existieren in 13 Bundesländern und variieren bezüglich Dauer (zwischen 5 und 37 Tagen) und Inhalt. Derzeit bestehen also auch keine länderübergreifenden Standards zur Qualifizierung oder zum Profil von Schulleitung.

Ähnlich wie in Deutschland werden auch in den USA die Heterogenität der Inhalte und eine schwankende Qualität der Ausbildung kritisiert, und ähnlich wie in Deutschland existieren dort (noch) keine nationalen Schulleitungsausbildungsstandards (Darling-Hammond, Meyerson, LaPointe \& Orr, 2009; Grissom, Mitani \& Woo, 2018). Im Gegensatz zu Deutschland ist jedoch eine Ausbildung vor Stellenantritt deutlich verbreiteter und findet i.d.R. an Hochschulen statt. Zur Führungskräfteentwicklung für alle Lehrkräfte, nicht nur jene, die später eventuell Schulleiter*innen werden, finden sich in den USA außerdem bereits Anregungen in vielen Lehramtsprogrammen. In einigen Bundesstaaten ist es möglich, während des Lehramtsstudiums optional ein Programm rund um schulische Führung zu belegen (siehe z. B. University of Richmond, 2018). Darüber hinaus bieten die Studienprogramme i.d.R. Platz für Wahlkurse (sog. electives). Sucht man in Deutschland in den Modulhandbüchern für die Bildungswissenschaften im Lehramt nach entsprechenden Themen oder Kompetenzformulierungen - wir haben das bei einigen exemplarisch getan -, kann man beispielsweise weder an den Hochschulen in Hamburg, Köln, Rostock, Regensburg, Halle oder Freiburg derartige Kurse oder Wahloptionen finden, wenngleich nicht ausgeschlossen werden kann, dass Themeninitiativen hier$\mathrm{zu}$ an anderen Hochschulen existieren oder von einzelnen Hochschullehrenden eingebracht werden.

Im Vorbereitungsdienst wiederum lassen sich exemplarisch am Beispiel BadenWürttembergs erste Konkretisierungen in Bezug auf personalführungs- und managementbezogene Fragen ausmachen. Beispielsweise findet man im Ausbildungsplan im Bereich der Schulkunde einen ersten Kenntnisaufbau zu Aspekten wie „Schulleitung: kollegiales, direktoriales Prinzip“ (Seminar für Ausbildung und Fortbildung Karlsruhe (Gymnasium), 2020, S. 19), und auch einige Verwaltungsaufgaben wie z. B. Konferenzen, Teambildung oder Organisationsweitergabe werden genannt, die im Bereich von Managementaufgaben zu verorten sind (ebd., S. 20). Bei einem Vergleich mit dem Kerncurriculum des Vorbereitungsdienstes in Nordrhein-Westfalen zeigt sich allerdings auch, dass sich die Ausrichtung von Bundesland $\mathrm{zu}$ Bundesland unterscheidet. Hier werden lediglich wieder primär teambezogene Kooperationen (peer- 
bezogen) ausgeführt (Kerncurriculum für den Vorbereitungsdienst in NordrheinWestfalen, MSW NRW, 2020, S. 9).

Solche ersten Spuren der Führungsthematik in den Ausbildungscurricula können als Zeichen gedeutet werden, dass ein Bewusstsein für diesen Bereich der Kompetenzentwicklung bereits im Ausbildungsstadium wächst, Personalführungs- und Managementperspektiven aber bislang noch nicht verbindlich expliziert sind. Ebenso als erster Schritt und dennoch nur als eine vereinzelte Initiative kann das Förderprogramm der Führungskräfteentwicklung für Lehramtsstudierende der Robert Bosch Stiftung (Baer \& Gleibs, 2013) gesehen werden, das initiiert wurde, weil auch die Kolleginnen dort die Führungskräfteentwicklung als einen vernachlässigten Bereich der ersten Phase der Lehrerinnenbildung wahrgenommen haben (Schneider \& Huber, 2015). Von einem systematisch angelegten Kompetenzaufbau für alle Lehrkräfte kann derzeit noch nicht gesprochen werden. Diesen jedoch einheitlich auf Hochschulniveau bereits über die Formulierung von Kompetenzstandards vorzusehen und in die Pflichtlehre zu integrieren, würde, so unsere These, Lehramtsstudierende auf Aufgaben vorbereiten, für die sie vom ersten Tag in der Schule Mitverantwortung tragen. Außerdem würden zwei weitere Möglichkeiten eröffnet: Alle angehenden Lehrkräfte lernten Möglichkeiten und Anforderungen potenzieller Karrierewege ihres Berufes kennen. Darüber hinaus hätten sie Gelegenheit zum Aufbau von Fähigkeiten, die sie für die Erfüllung von an sie delegierten Aufgaben im erweiterten Verantwortungsfeld der Führung benötigen. Dies soll nachfolgend weiter ausgeführt werden.

\section{Begründungen für die Aufnahme von Themen rund um Personalführung und Management ins Lehramtsstudium}

Die Lehramtsausbildung sieht vor, dass Studierende ihre Berufsrolle reflektieren und sich der besonderen Anforderungen des Berufsfeldes bewusst sind (KMK-Standards, „Innovieren“, 2004, S. 13f.). Dass Lehrer*in zu sein nicht nur Unterrichten, teamorientiertes Kooperieren und Beteiligung an schulentwicklungsbezogenen Aktivitäten bedeutet, sondern auch die Eingebundenheit in ein Mitarbeiter ${ }^{\star}$ innenverhältnis zu einer Schulleitung (zu einer Einzelperson und i.d.R. zu einem Leitungsteam, alleine oder gemeinsam mit dem Kollegium, vgl. Kansteiner-Schänzlin, 2002), wird Lehramtsstudierenden nicht gezielt vor Augen geführt, und anders als zu Unterricht liegen ihnen darüber auch keine Erfahrungen aus ihrer eigenen Schulzeit vor. Die Reflexion der beruflichen Entscheidung bleibt ohne diese Wahrnehmung jedoch eingeschränkt, sowohl im Hinblick auf bedeutsame Alltagsbedingungen als auch auf ihre Mitverantwortung dafür, dass diese beruflichen Beziehungen gelingen. ${ }^{3}$

3 Anders hingegen ist die Zusammenarbeit mit Eltern sowie ihre Beratung mehrfach expliziert (KMK, 2004, S. 3, 10, 11). 
Außerdem bleiben potenzielle Karrierewege im Rahmen ihrer Berufsentscheidung intransparent. Die möglichen Funktionen und die dort zu verantwortenden Aufgaben erschließen sich ihnen erst mit einiger Berufserfahrung. Der Bewerber*innenmangel legt jedoch nahe, viel früher die anderen potenziellen Aufgabenfelder im Schulkontext sichtbar und selbstverständlich werden zu lassen.

Darüber hinaus erscheint eine Kompetenzentwicklung im Bereich Personalführung sinnvoll, da Lehrkräfte in zahlreiche Führungssituationen eingebunden sind und in diesen variabel reagieren müssen. Bilanziert man derzeitig favorisierte Führungskonzepte, findet man schulische Führungskonzepte von lenkend-einbindend bis kooperativ. Aktuell diskutierte Führungsstile fordern Mitarbeiter*innen recht unterschiedlich heraus: Die transformationale Führung legt die Lenkleistung stark in die Hand der Führungsperson, bindet dann jedoch Mitarbeiter*innen mit ein (u.a. Northouse, 2016; Furtner, 2016), während die kooperative, partizipative oder demokratische Führung von vornherein stärker eine Mitverantwortung aller Lehrkräfte bei Entscheidungen vorsieht (u.a. Wunderer, 2009; Schmidt \& Richter, 2009). Bei Instructional Leadership (u.a. Wissinger, 2011; Klein, 2018) wird Führung wiederum stark auf Unterrichtsentwicklung und damit den „üblichen Hoheitsraum“ von Lehrkräften hin angelegt oder bei Distributed Leadership Führung gar auf mehrere Personen aufgeteilt und somit von verschiedenen Personen ausgebracht (u.a. Hallinger \& Heck, 2010; Werther, 2014). Überdies wird je nach Situation eine Mischung der Stile empfohlen (Huber \& Rolff, 2010; Pellny, Schmelcher \& Beinlich, 2014). Mitarbeiter ${ }^{\star}$ innen müssen folglich flexibel mit unterschiedlichem Führungsverhalten und verschiedenen Personen, die Führungsimpulse setzen, rechnen und situativ damit umgehen. Da Führung keine Einbahnstraße ist (Stuke, 2015; GieskeRoland, 2015), sondern für das Gelingen beide, sowohl Führungskräfte als auch Mitarbeiter*innen, verantwortlich sind, lässt sich die Notwendigkeit ableiten, auch Lehrkräfte bereits im Studium zum Aufbau von Kompetenz in Fragen der Personalführung zu begleiten.

Eingebunden in Personalführungs- bzw. Managemententscheidungen sind Lehrkräfte auch, wenn ihnen Aufgaben der Schulleitung übertragen werden. Für deren Erfüllung müssen sie die entsprechenden Kompetenzen zumindest auf einem soliden Niveau bereits entwickelt haben und können nicht erst Fortbildungen besuchen, wenn ihnen eine Aufgabe übertragen wurde. Die im Rahmen der Delegation an Lehrkräfte übergehende Verantwortung umfasst auch Aufgaben wie die Planung, Durchführung und Ergebnissicherung (einschließlich Controlling) innerschulischer und außerschulischer Kooperationen (z. B. Leitung von Fachkonferenzen oder regionalen Konferenzen zum Übergang Kita-Schule), Personalentwicklung (z.B. Ausbringen schulinterner Fortbildung, Leitung Professioneller Lerngemeinschaften) (Stamann \& Kansteiner, 2014) sowie die Steuerung von Schulentwicklung auf Organisationsebene (Rolff, 2010) einschließlich des Einsatzes datengestützter Steuerung (Schratz et al., 2015) (z. B. Steuergruppenmitwirkung, Arbeitszeitorganisation von Lehrkräfteteams). 
Die Mitverantwortung an derlei Aufgaben fällt u.E. in den Bereich einer Führung, die Schulentwicklung ansteuert. In nicht geringem Maße zielt sie darauf ab, bestmögliche Bedingungen für Lehren und Lernen auf allen Ebenen zu schaffen und damit nicht nur für das Funktionieren der Einzelschule zu sorgen, sondern auch für deren Weiterentwicklung. Diese Zielperspektive konstituiert die Notwendigkeit und Legitimität einer „absichtsvolle[n] soziale[n] Einflussnahme von Personen auf andere Personen" (Wegge \& von Rosenstiel, 2014, S. 316). Wenn Führung und Schulentwicklung derart miteinander verknüpft sind, dann spricht dies nicht zuletzt auch dafür, nicht nur eines der beiden im Zielfokus von Kompetenzstandards in der Ausbildung zu verankern.

Bereits in der ersten Phase der Lehrer*innenbildung dazu systematisch Kenntnisse aufzubauen und Bedingungen zu reflektieren, heißt nicht zuletzt, den Verantwortungsraum aus verschiedenen Rollen heraus abschätzen zu lernen. Eine umfassendere Reflexion des Berufswunsches und möglicher Karrierewege, die Vorbereitung auf den beruflichen Start (bereits ab dem Referendariat) mit seinen zentralen Beziehungskonstellationen und Herausforderungen (Keller-Schneider, 2019, 2020; Weiß, Lerche \& Kiel, 2016) sowie die Vorbereitung auf die Übernahme schulischer Aufgaben würden einen wichtigen Beitrag im Feld der Führungskräfteentwicklung leisten.

\section{Lücken in den Standards und Kompetenzmodellen/-katalogen}

Sichtet man exemplarisch derzeit genutzte Standards und Kompetenzmodelle bzw. Kompetenzkataloge der Lehrer*innenbildung, sieht man auch hier Lücken, wie bereits für die KMK-Standards gezeigt. In der für die Diskussion um Kompetenzstandards maßgeblichen Studie COACTIV (Kunter, Baumert, Blum, Klusmann, Krauss \& Neubrand, 2011) wurde der Kompetenzbereich Organisationswissen/Interaktionswissen zwar theoretisch unter das Konzept des bildungswissenschaftlichen Wissens subsummiert, allerdings auch (nur) am Beispiel „Teamfähigkeit“ expliziert. Die Oserschen Standards (Oser, 1997) tangieren in Standardgruppe 9, „Zusammenarbeit in der Schule“, nur die Verteilung der Kompetenzen zwischen Schulaufsicht, Schulleitung und Lehrer*innenschaft, wie Konflikte in diesem Bereich bearbeitet werden können und wie man die zur Verfügung stehenden finanziellen Mittel sinnvoll einsetzt. Führungskonzeptionen, konstruktives Verhalten in Führungsbeziehungen o. ä. werden nicht thematisiert.

Konzeptionell hingegen hat das europäische Tuning-Projekt (Gonzales \& Wagenaar, 2003) Personalführungs- und Managementkompetenz miterfasst. Es legt für die Erhebung von Kompetenzen in verschiedenen Studiengängen das Augenmerk u.a. auf überfachliche Kompetenzen, und hier interessieren in ihrer Erhebung Fähigkeiten wie Mitarbeiter*innenführung sowie Projektgestaltung und -management. 
Folgt man der hier aufgezeigten Überlegung, nach der bereits in der ersten Phase der Lehrer*innenbildung der Aufbau personalführungs- und managementbezogener Kompetenzen anzustreben ist, scheint ein Blick in Studiengänge vielversprechend, die als Weiterbildungsstudiengänge für die dritte Phase der Lehrer*innenbildung konzipiert wurden und Aspekte der Führungskräfteentwicklung abdecken. Im Rahmen einer Vollerhebung (Krüger, 2016) konnten im deutschsprachigen Raum 51 solcher Masterstudiengänge identifiziert werden; neun von diesen adressierten explizit und ausschließlich den schulischen Sektor (kurz Schulmanagement-Studiengänge).

Bilanziert man die Studie nach zwei Kategorien, die Führung grundsätzlich ausmachen - personale Führung (Führung des Menschen durch den Menschen) und strukturelle Führung oder Management (Führung des Menschen durch Strukturen) (Wunderer, 2009; ähnlich Decker, 1995) -, so fällt auf, dass in BildungsmanagementStudiengängen häufiger zu personaler Führung gelehrt wird (insgesamt $25 \%$ ) als zu struktureller Führung, die dem Management gleichgesetzt werden kann (14\%). Innerhalb der Teilgruppe der Schulmanagement-Studiengänge ist dieser Trend sogar noch ausgeprägter. Aufgaben der strukturellen Führung und damit eher managementbezogene Aufgaben wie beispielsweise die klare Koordinierung von Gremienzuständigkeiten, die Abstimmung organisationaler Kommunikations- und Entscheidungswege oder die Entwicklung und Bewertung von Strategien werden vergleichsweise geringfügig behandelt. Da beide Führungsstränge gleichermaßen bedeutsam sind, machen diese Befunde darauf aufmerksam, bei der Aufnahme von Personalführung und Management in das Lehramtsstudium beide Führungsdimensionen hinreichend zu berücksichtigen.

\section{Fazit}

Im vorliegenden Beitrag haben wir für die Aufnahme des Themenfelds Personalführung und Management in die Lehramtsausbildung argumentiert, weil angehende Lehrkräfte grundsätzlich über entsprechende Kompetenzen verfügen müssen, sollen sie in den Führungskonstellationen als Mitarbeiter*innen zum Gelingen beitragen und delegierte Aufgaben erfolgreich bewerkstelligen. Der frühe Kompetenzaufbau stellt zugleich eine Investition in die Führungskräfteentwicklung dar, da Karrierewege antizipiert und der Verantwortungsraum umfassend reflektiert werden können.

Unbenommen der hier vorgebrachten Argumente ist zu berücksichtigen, dass eine solche curriculare Erweiterung aufgrund der zumindest derzeit nicht möglichen zeitlichen Erweiterung des Studienumfangs eine thematische Verdrängung im Studium nach sich ziehen würde. Inwiefern dies auch Auswirkungen auf den Erwerb anderer als zentral erachteter Kompetenzen hätte, ist zum jetzigen Zeitpunkt eine offene Frage, ebenso wie jene danach, wie Lernarrangements aussehen könnten, die dem 
hier dargelegten Anspruch Rechnung tragen. Zudem sehen Schulpraktika aktuell nicht vor, dass Studierende Personalführungs- und Managementaufgaben systematisch explorieren. In der Konsequenz läge hier eine kaum oder nur punktuell ausgeprägte, in Summe aber unsystematische Theorie-Praxisanbindung vor. Eine curriculare Erweiterung müsste, würden Personalführungs- und Managementkompetenzen entsprechend unserer These als bedeutsam aufgenommen, nicht nur über alle Stränge des Lehramtsstudiums hinweg sinnvoll verknüpft, sondern auch passend in die Modulhandbücher eingeflochten werden. Ihre Aufnahme in die Kompetenzstandards würde dies vorbereiten und absichern.

\section{Literatur und Internetquellen}

Arnold, R., Bonsen, M., Huber, S. G., Schratz, M., \& Rolff, H.-G. (2010). Führung, Steuerung, Management. Orientierungsband. Seelze: Klett.

Baer, M., \& Gleibs, H. E. (2013). Führungskompetenzen angehender Lehrerinnen und Lehrer stärken. In S. G. Huber (Hrsg.), Führungskräfteentwicklung. Grundlagen und Handreichungen zur Qualifizierung und Personalentwicklung im Schulsystem (S. 187194). Köln: Carl Link.

Bonsen, M. (2009). Wirksame Schulleitung. In H. Buchen \& H.-G. Rolff (Hrsg.), Professionswissen Schulleitung (S. 193-228) (2., erw. Aufl.). Weinheim: Beltz.

Bonsen, M., \& Berkemeyer, N. (2014). Lehrerinnen und Lehrer in Schulentwicklungsprozessen. In E. Terhart, H. Bennewitz \& M. Rothland (Hrsg.), Handbuch der Forschung zum Lehrerberuf (S. 920-931). Münster: Waxmann.

Buchen, H., \& Rolff, H. G. (Hrsg.). (2006). Professionswissen Schulleitung. Weinheim: Beltz.

Covey, S. (2007). The Transformational Leadership Report. Zugriff am 10.01.2017. Verfügbar unter: http://www.transformationalleadership.net/products/TransformationalLeader shipReport.pdf.

Cramer, C., Gross Ophoff, J., Pietsch, M., \& Tulowitzki, P. (2020). Schulleitung in Deutschland: zentrale Ergebnisse einer repräsentativen Studie. Schulmanagement: Zeitschrift für Schulleitung und Schulpraxis, (5), 17-20.

Darling-Hammond, L., Meyerson, D., LaPointe, M., \& Orr, M. T. (2009). Preparing Principals for a Changing World: Lessons From Effective School Leadership Programs. San Francisco, CA: Jossey-Bass. https://doi.org/10.1002/9781118269329

Decker, F. (1995). Bildungsmanagement. Lernprozesse erfolgreich gestalten pädagogisch und betriebswirtschaftich führen, budgetieren und finanzieren. München: AOL-Verlag \& Lexika-Verlag.

Easley, J., \& Tulowitzki, P. (2013). Policy Formation of Intercultural and Globally Minded Educational Leadership Preparation. International Journal of Educational Management, 27 (7), 744-761. https://doi.org/10.1108/IJEM-04-2012-0050

Fullan, M. (2007). The New Meaning of Educational Change. New York: Teachers College Press.

Furtner, M. (2016). Effektivität der Transformationalen Führung. Helden, Visionen und Charisma. Wiesbaden: Springer Gabler. https://doi.org/10.1007/978-3-658-15321-2

Gieske-Roland, M. (2015). Mikropolitik und schulische Führung. In J. Berkemeyer, N. Berkemeyer \& F. Meetz (Hrsg.), Professionalisierung und Schulleitungshandeln. Wege und Strategien der Personalentwicklung an Schulen (S. 33-51). Weinheim: Beltz Juventa.

Gonzales, J., \& Wagenaar, R. (2003). Universities' Contribution to the Bologna Process. Bilbao: Publicaciones de la Universidad de Deusto. 
Katja Kansteiner, Pierre Tulowitzki, Michael Krüger \& Christoph Stamann

Grissom, J. A., Mitani, H., \& Woo, D. S. (2018). Principal Preparation Programs and Principal Outcomes. Educational Administration Quarterly, 1-43. https://doi.org/10. 1177/0013161X18785865

Hallinger, P., \& Heck, R. H. (2010). Leadership for Learning: Does Collaborative Leadership Make a Difference in School Improvement? Educational Management Administration \& Leadership, 38 (6), 654-678. https://doi.org/10.1177/1741143210379060

Hancock, D., \& Müller, U. (2012). Identifying Factors That Influence the Motivation of German and U.S. Teachers to Become School Principals. In R. Nata (Hrsg.), Germany: Social, Economic and Political Developments (S. 299-306). Hauppauge, NY: Nova Science Publishers.

Harris, A. (2010). Distributed Leadership: Evidence and Implications. In T. Bush, L. Bell \& D. Middlewood (Hrsg.), The Principles of Educational Leadership \& Management (2. Aufl.) (S. 55-69). London: Sage. https://doi.org/10.1007/978-1-4020-9737-9

Heinrich, M., \& Altrichter, H. (2008). Schulentwicklung und Profession. Der Einfluss von Initiativen zur Modernisierung der Schule auf die Lehrerprofession. In W. Helsper, S. Busse, M. Hummrich \& R.-T. Kramer (Hrsg.), Pädagogische Professionalität in Organisationen: Neue Verhältnisbestimmungen am Beispiel der Schule (S. 205-224). Wiesbaden: VS. https://doi.org/10.1007/978-3-531-90777-2_11

Huber, S. G., \& Rolff, H.-G. (2010). Delegation und System Leadership. In H.-G. Rolff (Hrsg.), Führung, Steuerung, Management (S. 43-58). Seelze: Kallmeyer.

Huber, S. G., \& Schwander, M. (2015). Das Kompetenzmodell für pädagogische Führung. In S. G. Huber, Stiftung der Deutschen Wirtschaft \& Robert Bosch Stiftung (Hrsg.), Schule gemeinsam gestalten - Entwicklung von Kompetenzen für pädagogische Führung (S. 17-51). Münster \& New York: Waxmann.

Huber, S. G., Wolfgramm, C., \& Kilic, S. (2013). Vorlieben und Belastungen im Schulleitungshandeln: Ausgewählte Ergebnisse aus der Schulleitungsstudie 2011/2012 in Deutschland, Österreich, Liechtenstein und der Schweiz. In S. G. Huber (Hrsg.), Jahrbuch Schulleitung 2013 (S. 259-271. Köln: Wolters Kluwer.

Kansteiner, K. (2019). Professionelle Lerngemeinschaften. Perspektiven auf die entwicklungsorientierte Kooperation von Lehrkräften und Schulleitungen. SchulmanagementHandbuch, 40 (172), München: Oldenbourg.

Kansteiner, K., \& Klose, O. (2017). Schulleitungsbesetzung unter der integrierenden Perspektive von Gender Mainstreaming und Diversity Management. Abschlussbericht zum Forschungsprojekt. Zugriff am 14.02.2020. Verfügbar unter: https://www.gew.de/index. php? eID $=$ dumpFile \&t $=\mathrm{f} \& \mathrm{f}=75954 \&$ token $=\mathrm{b} 66454877 \mathrm{f} 7 \mathrm{~b} 970695136055 \mathrm{bfd} 5 \mathrm{ac50b} 1 \mathrm{dcd}$ $47 \mathrm{f} \&$ sdownload $=\& \mathrm{n}=$ Kansteiner_Klose_Abschlusbericht_Schulleitungsbesetzungsver fahren_GMDM_FINAL.pdf.

Kansteiner-Schänzlin, K. (2002). Personalführung in der Schule - Übereinstimmungen und Unterschiede zwischen Frauen und Männern in der Schulleitung. Bad Heilbrunn: Klinkhardt.

Keller-Schneider, M. (2019). Professionalisierung im Berufseinstieg von Lehrpersonen: Individuelle Wahrnehmung - institutionelle Angebote - berufsphasenspezifische Herausforderungen und Ressourcen. In M. Syring \& S. Weiß (Hrsg.), Lehrer(in) sein - Lehrer(in) werden - die Profession professionalisieren (S. 145-160). Bad Heilbrunn: Klinkhardt.

Keller-Schneider, M. (2020). Entwicklungsaufgaben im Berufseinstieg von Lehrpersonen. Bearbeitung von beruflichen Herausforderungen im Zusammenhang mit Kontext- und Persönlichkeitsmerkmalen und in berufsphasendifferenten Vergleichen (2., überarb. u. erw. Aufl.). Münster: Waxmann.

Klein, E. D. (2018). Schulleitungen als Instructional Leader. Pädagogische Führung, 29 (5), 178-180. 
Führungskräfteentwicklung in der Lehramtsausbildung |

KMK (Sekretariat der Ständigen Konferenz der Kultusminister der Länder in der Bundesrepublik Deutschland). (2004/i.d.F. 2019). Standards für die Lehrerbildung: Bildungswissenschaften. Zugriff am 11.04.2021. Verfügbar unter: https://www.kmk.org/ fileadmin/Dateien/veroeffentlichungen_beschluesse/2004/2004_12_16-Standards-Leh rerbildung-Bildungswissenschaften.pdf.

Krüger, M. (2016). Das Lernwissen der Bildungsmanagement-Studiengänge im deutschsprachigen Raum. Seine Entstehung sowie seine Formen und Auswirkungen. Hamburg: Dr. Kovač.

Kunter, M., Baumert, J., Blum, W., Klusmann, U., Krauss, S., \& Neubrand, M. (Hrsg.). (2011). Professionelle Kompetenz von Lehrkräften - Ergebnisse des Forschungsprogramms COACTIV. Münster: Waxmann.

Lortie, D. C. (1972). Team Teaching - Versuch der Beschreibung einer zukünftigen Schule. In H. W. Dechert (Hrsg.), Team Teaching in der Schule (S. 37-76). München: Piper.

MKJS BW (Ministerium für Kultus, Jugend und Sport Baden-Württemberg). (2016). Das Anforderungsprofil Schulleiterinnen und Schulleiter. Zugriff am 30.03.21. Verfügbar unter: https://km-bw.de/,Lde/Startseite/Schule/Anforderungsprofil+Schulleitungen.

MSW NRW (Ministerium für Schule und Weiterbildung des Landes Nordrhein-Westfalen). Kerncurriculum für den Vorbereitungsdienst in Nordrhein-Westfalen (2020). Zugriff am 23.01.2020. Verfügbar unter: https://www.schulministerium.nrw.de/docs/bp/Lehrer/ Lehrkraft-werden/Vorbereitungsdienst/index.html.

Northouse, P. (2016). Leadership. Theory and Practice. Los Angeles, CA: Sage.

Organization for Economic Cooperation and Development (OECD). (Hrsg.). (2008). Improving School Leadership, Bd. 1: Policy \& Practice. Paris: OECD.

Oser, F. (1997). Standards in der Lehrerbildung. Beiträge zur Lehrerbildung, 15 (2), 26-37.

Pellny, M., Schmelcher, J., \& Beinlich, A. (2014). Führungskompetenz. Was wirklich wichtig ist. Erlangen: Publicis.

Rolff, H. G. (Hrsg.). (2010). Führung, Steuerung, Management. Seelze: Kallmeyer.

Rothland, M., Biederbeck, I., Grabosch, A., \& Heiligtag, N. (2017). Das Autonomie-ParitätsMuster bei Lehramtsstudierenden. Lehrerbildung auf dem Prüfstand, 10 (2), 195-215.

Schmidt, B., \& Richter, A. (2009). Zwischen Laissez-Faire, Autokratie und Kooperation: Führungsstile von Professorinnen und Professoren. Beiträge zur Hochschulforschung, 31 (4). Zugriff am 21.01.2017. Verfügbar unter: http://www.bzh.bayern.de/uploads/ media/4-2009-schmidt-richter.pdf.

Schneider, N., \& Huber, S. G. (2015). Beispiele aus der Lehrerbildung zur Entwicklung von Kompetenzen für pädagogische Führung und Schulentwicklung. Ausgewählte Ergebnisse einer explorativen Befragung. In S. G. Huber, Stiftung der Deutschen Wirtschaft \& Robert Bosch Stiftung (Hrsg.), Schule gemeinsam gestalten - Entwicklung von Kompetenzen für pädagogische Führung. Beiträge zu Leadership in der Lehrerbildung (S. 109-132). Münster \& New York: Waxmann.

Schratz, M., Wiesner, C., Kemethofer, D., George, A. C., Rauscher, E., Krenn, S., \& Huber S. G. (2015). Schulleitung im Wandel: Anforderungen an eine ergebnisorientierte Führungskultur. In M. Bruneforth, F. Eder, K. Krainer, C. Schreiner, A. Seel \& C. Spiel (Hrsg.), Nationaler Bildungsbericht Österreich 2015, Bd. 2: Fokussierte Analysen bildungspolitischer Schwerpunktthemen (S. 221-262). Graz: Leykam. http://dx.doi. org/10.17888/nbb2015-2-6.

Seminar für Ausbildung und Fortbildung der Lehrkräfte Karlsruhe (Gymnasium) (2020). Ausbildungsplan. Zugriff am 23.01.2020. Verfügbar unter: http://gym.seminar-karlsruhe. de/Lde/Startseite/Referendariat/Ausbildungsplan.

Senator für Arbeit, Frauen, Gesundheit, Jugend und Soziales der Hansestadt Bremen (2005). Führen - Leiten - Zukunft gestalten in Schulen für Gesundheitsfachberufe. Ein Anforde- 
Katja Kansteiner, Pierre Tulowitzki, Michael Krüger \& Christoph Stamann

rungsprofil für Schulleitungen. Zugriff am 03.01.2017. Verfügbar unter: https://www. wisoak.de/fileadmin/user_upload/Schulleitung_Anforderungsprofil-1.pdf.

Stamann, C., \& Kansteiner, K. (2014). Die strategische Gestaltung der Personalentwicklung. In E. Steger Vogt, K. Kansteiner \& M. Pfeifer (Hrsg.), Gelingende Personalentwicklung in der Schule (S. 55-74). Innsbruck: StudienVerlag.

Stuke, T. (2015). Mit Werten führen. Bad Heilbrunn: Klinkhardt.

Strauss, N.-C., \& Anderegg, N. (Hrsg.). (2020). Teacher Leadership - Schule gemeinschaftlich führen. Bern: hep.

Thom, N., Ritz, A., \& Steiner, R. (2002). Effektive Schulführung. Chancen und Gefahren des Public Managements im Bildungswesen (2. Aufl.). Bern: Haupt.

Tulowitzki, P., Hinzen, I., \& Roller, M. (2019). Die Qualifizierung von Schulleiterinnen und Schulleitern in Deutschland - ein bundesweiter Überblick. DDS - Die Deutsche Schule, 111 (2), 149-170. https://doi.org/10.31244/dds.2019.02.04

Tulowitzki, P., \& Pietsch, M. (2020). Stichwort: Lernzentriertes Leitungshandeln an Schulen - Leadership for Learning. Zeitschrift für Erziehungswissenschaft, 23, 873-902. https:// doi.org/10.1007/s11618-020-00964-8

University of Richmond. (2018). Lead I - Center for Leadership in Education. Zugriff am 17.09.2018. Verfügbar unter: https://spcs.richmond.edu/professional-education/areas/ teaching-instruction/leadership-education/lead-one.html.

Wegge, J., \& von Rosenstiel, L. (2014). Führung. In H. Schuler \& K. Moser (Hrsg.), Lehrbuch Organisationspsychologie (S. 315-367). Bern: Hans Huber.

Weiß, S., Lerche, T., \& Kiel, E. (2016). Die zweite Ausbildungsphase in Deutschland aus der Sicht von Lehramtsreferendarinnen und Lehramtsreferendaren im Kontext von Gestaltungsmöglichkeiten und Anpassungsnotwendigkeiten. In J. Košinàr, S. Leineweber \& E. Schmid (Hrsg.), Professionalisierungsprozesse angehender Lehrpersonen in den berufspraktischen Studien (S. 119-137). Münster: Waxmann.

Werther, S. (2014). Geteilte Führung. Ein Überblick über den aktuellen Forschungsstand. Wiesbaden: Springer Gabler. https://doi.org/10.1007/978-3-658-05344-4

Wissinger, J. (2011). Schulleitung und Schulleitungshandeln. In E. Terhart, H. Bennewitz \& M. Rothland (Hrsg.), Handbuch der Forschung zum Lehrerberuf (2. Aufl.) (S. 98-115). Münster: Waxmann.

Wunderer, R. (2009). Führung und Zusammenarbeit. Eine unternehmerische Führungslehre (8., aktual. u. erw. Aufl.). Berlin: Luchterhand.

Katja Kansteiner, Prof. Dr., geb. 1968, Professorin für Erziehungswissenschaft an der Pädagogischen Hochschule Weingarten.

E-Mail: kks@ph-weingarten.de

Korrespondenzadresse: PH Weingarten, Kirchplatz 2, 88250 Weingarten

Pierre Tulowitzki, Prof. Dr., geb. 1982, Leiter der Professur für Bildungsmanagement und Schulentwicklung an der Pädagogischen Hochschule der Fachhochschule Nordwestschweiz.

E-Mail: pierre.tulowitzki@fhnw.ch.

Korrespondenzadresse: PH FHNW, Bahnhofstrasse 6, 5210 Windisch, Schweiz

Michael Krüger, Dr., geb. 1971, Akademischer Rat an der Abteilung für internationales Bildungsmanagement an der Pädagogischen Hochschule Ludwigsburg.

E-Mail: krueger@ph-ludwigsburg.de

Korrespondenzadresse: PH Ludwigsburg, Reuteallee 46, 71634 Ludwigsburg 
Christoph Stamann, M. A., geb. 1984, Akademischer Mitarbeiter im Projekt Leistung macht Schule (LemaS) am Institut für Allgemeine und Historische Erziehungswissenschaft an der Pädagogischen Hochschule Karlsruhe.

E-Mail: christoph.stamann@ph-karlsruhe.de

Korrespondenzadresse: PH Karlsruhe, Bismarckstraße 10, 76133 Karlsruhe 
Martin Rothland

\title{
Die „Lehrerpersönlichkeit“: das Geheimnis des Lehrberufs?
}

\section{Zusammenfassung}

Die ungebrochene Popularität der Idee der "Lehrerpersönlichkeit" beschränkt sich nicht - wie angenommen wird - auf die Selbstentwürfe berufserfahrener Lehrer*innen. Vielmehr offenbaren auch der neuere akademische Lehrer ${ }^{*}$ nnenbildungsdiskurs ebenso wie die wissenschaftliche Diskussion zum Lehrberuf eine erstaunliche Beharrlichkeit des Konstruktes. Die "Lehrerpersönlichkeit“ wird mit der Begründung, dass sie ausschlaggebend für den Lernerfolg der Schüler*innen sei, als Ziel der Lehrer*innenbildung und Professionalisierung als Persönlichkeitsbildung entworfen und gefordert. Zugleich sei die "Lehrerpersönlichkeit“ aber nicht recht bestimmbar, nicht zu operationalisieren oder zu messen. Sie erscheint vielmehr als universelle Antwort auf das, was im Lehrberuf nicht erklärbar und nicht empirisch rekonstruierbar ist. Was aber nicht zu bestimmen ist, was nicht (be)greifbar erscheint, das kann nicht erlernt und vermittelt werden. Insofern ist die Forderung, Professionalisierung für und Professionalität im Lehrberuf an die Ausbildung der „Lehrerpersönlichkeit“ zu knüpfen, gar nicht einzulösen.

Schlüsselwörter: Lehrer*innenbildung, Lehrberuf, Lehrerpersönlichkeit, Professionalisierung, Professionalität

\section{The “Teacher Personality": Secret of the Teaching Profession?}

\begin{abstract}
The popularity of the idea of a "teacher personality" is not limited to the self-awareness of experienced teachers. The newer academic discourse on teacher education, as well as the academic discussion on the teaching profession, reveal an astonishing persistence of the construct. Because of its assumed decisiveness for the learning success of pupils, the "teacher personality" is designed and demanded as the goal of teacher education and professionalization as personality development. At the same time, the "teacher personality" seems indeterminable, as if it can neither be operationalized nor measured. Rather, it appears as a universal answer to what cannot be explained or empirically reconstructed about the teaching profession. But what cannot be determined, what does not seem to be comprehensible, can hardly be learned or taught. In this respect, the demand to link
\end{abstract}


professionalization for and professionalism in the teaching profession to the training of the "teacher personality" cannot be met at all.

Keywords: teacher education, teaching profession, teacher personality, professionalization, professionalism

\section{1 „Lehrerpersönlichkeit“ im (Lehrerinnenbildungs-) Diskurs}

Ist die „Lehrerpersönlichkeit“ in neueren Handbuch- oder Studienbuchbeiträgen Gegenstand der Erörterungen, so wird darauf verwiesen, dass das Konstrukt (1.) insbesondere im Selbstverständnis der „Praktiker“ bedeutsam sei (vgl. Bromme \& Haag, 2008; Haag, 2013). Berufserfahrene und -zufriedene Lehrer*innen führen selbst ihren Berufserfolg bzw. generell Professionalität auf den Faktor „Lehrerpersönlichkeit“ zurück. Sie verstehen unter diesem Begriff „ein Ensemble von Eigenschaften, die erstens zentral für eine erfolgreiche Berufsausübung sind, sich zweitens nicht trennscharf umreißen lassen und drittens den Charakter des ,Nichterlernbaren" tragen" (Herrmann \& Hertramph, 2002, S. 203). Das bedeutet, Lehrer*innen, die sich durch umfassende Erfahrung in ihrem Beruf auszeichnen, erklären, dass zentrale Momente ihres Berufs nicht erlernbar sind. Sie müssen ihnen damit qua Veranlagung als besondere Gabe oder Talent gegeben sein.

Unter der „Lehrerpersönlichkeit“ wird von den Lehrkräften selbst häufig „der Charakter, eine Respektsperson verstanden, und dieses Wort steht als Synonym für eine Wesensart, eine Veranlagung und eine Eigenart" (Fink-Pomberger \& Volkmer, 2015, S. 13). Gemeint sei mit „Lehrerpersönlichkeit“ die „persönliche Note, die authentische Ausstrahlung des Lehrers, also Eigenschaften, die sich auch noch im größten Getümmel des Unterrichts positiv bemerkbar machen" (Werner, 2013, S. 50). Gesprochen wird von natürlicher Autorität und einer charismatischen Persönlichkeit (ebd., S. 52). In neueren Forschungsbefunden bestätigt sich denn auch, dass von den Lehrer*innen die Ausstrahlung dieser natürlichen Autorität und das Zeigen innerer Stärke als persönliche Eigenschaften hervorgehoben werden, Eigenschaften, die - so die hier befragten Grundschullehrkräfte - nicht erlernbar seien (Weiß, Schramm \& Kiel, 2013).

Die Wesensmerkmale, die mit der „Lehrerpersönlichkeit“ verbunden werden, schließen ungebrochen an die Tradition geisteswissenschaftlicher Pädagogik an, die in idealisierenden Übertreibungen des Persönlichkeitskonzeptes in Gestalt von Tugendkatalogen ihren Ausdruck findet (Neuweg, 2008, S. 16). In dieser Tradition erscheint das Konstrukt „Lehrerpersönlichkeit" insofern als besonders bedeutsam, als dass Lehrer*innen nicht allein als Vermittler*innen von Kultur, sondern selbst als Kulturträger*innen, als personifizierte Kultur inklusive durch die Person vorbildlich und modellgebend repräsentierter Normen und Werte entworfen werden (Bürkler, 
Rosenmund \& Schmid, 2005; Rothland, Cramer \& Terhart, 2018). Die Rede von der „Lehrerpersönlichkeit“ ist hier zudem auch aktuell verbunden mit der Vorstellung einer Berufung für den Lehrberuf: „Eine Lehrperson zu sein bedeutet, sich dazu berufen zu fühlen“ (Draxler, 2015, S. 99).

Zweitens (2.) wird für den wissenschaftlichen Diskurs jenseits des Selbstverständnisses der „Praktiker“ konstatiert, „dass das Konzept der ,Lehrerpersönlichkeit“ wenig taugt, um hiermit Unterschiede des pädagogischen Einflusses erklären und vorhersagen zu können“ (Bromme \& Haag, 2008, S. 804; vgl. bereits Getzels \& Jackson, 1970). Der Begriff der „Lehrerpersönlichkeit“ spiele „spätestens seit der sogenannten realistischen Wende der Erziehungswissenschaft nur noch eine marginale Rolle in der wissenschaftlichen Debatte“ (Pfützner, 2013, S. 43).

Wie ein Blick in den neueren und neuesten Lehrer*innenbildungsdiskurs offenbart, reduzieren sich die Popularität der „Lehrerpersönlichkeit“ sowie die Rede von einer Berufung zur Lehrer*innenarbeit keinesfalls auf die Selbstentwürfe der Profession. Stattdessen wird an dem Konstrukt (spätestens) seit dem 19. Jahrhundert bis heute im deutschsprachigen Raum sowohl im Alltagsdiskurs als auch in der akademischen Diskussion festgehalten - u. a. verbunden mit der Frage: „Kann man alles, was man als Lehrer/in können sollte, lernen, oder muss man manches auch ,sein'?" (Neuweg, 2008, S. 15; vgl. Haag \& Streber, 2020, S. 165 ff.).

Bereits in einem Sammelband aus dem Jahr 1980 wird die Wiederentdeckung der Lehrerpersönlichkeit gefeiert (Paffrath, 1980, S. 11). Ulrich Herrmann verfolgt im Jahr 2000 zwar den langen „Abschied vom ,geborenen Erzieher“', verweist aber zugleich darauf, dass allein die Rede von Kompetenzen und Qualifikationen bezogen auf die Berufsfähigkeit von Lehrkräften ignoriere, dass es Persönlichkeitstypen gebe, die zu beachten seien, „wenn berufliche Profile angesonnen werden, die der/die Betreffende sich vielleicht habituell gar nicht zu Eigen machen kann“ (Herrmann, 2000, S. 18). Schließlich werden bspw. im journal für lehrerInnenbildung „Lehrerpersönlichkeit“ und Lehrer*innenbildung zusammengebracht:

„Lehrerinnen und Lehrer wirken mit ihrer ganzen Persönlichkeit. Insofern braucht es auch Arbeits- und Inszenierungsformen, welche die für die Lehrertätigkeit so wichtige Person der Handelnden schon in der Ausbildung in Szene setzen und präsent werden lassen“" (Messner, 2001, S. 12),

gilt doch die „Lehrerpersönlichkeit“ als "Grundlage aller pädagogischen Arbeit“ (Gudjons, 2013, S. $11 \mathrm{ff}$.).

Dass Lehrer*innen Kraft ihrer Persönlichkeit erziehen, suggeriert nach wie vor der gleichnamige Beitrag in der Neuausgabe des Studienbuchs Schulpädagogik (Haag, 2013, S. 366 ff.). Schließlich brauche es „starke Lehrerpersönlichkeiten, die „vor Klas- 
sen stehen“ und „überzeugend unterrichten können“ (Dubs, 2008, S. 11), und als Lehrkraft stehe man individuell ,immer in der Spannung zwischen Profession und Person“ (Röbe, Aicher-Jakob \& Seifert, 2019, S. 281). Die Rede von einer „Berufung“ ist im Lehrer*innenbildungsdiskurs ebenfalls aktuell zu vernehmen: Der Ansatz, den Lehrberuf nicht „lediglich als Beruf“ anzusehen, entspreche einer „kulturellen Notwendigkeit" (ebd., S. 290). Definitiv, so Klaus Zierer, sei der Lehrberuf nicht nur Beruf, sondern auch Berufung (Zierer in der 3sat-Dokumentation „Klasse Lehrer?", Erstausstrahlung 18. Oktober 2018).

Worauf kann zurückgeführt werden, dass die Rede von der "Lehrerpersönlichkeit" auch im wissenschaftlichen bzw. im Lehrer*innenbildungskurs alles andere als überwunden erscheint und stattdessen „der Bezugnahme auf die Lehrerpersönlichkeit ein ,wiederkehrender Zauber“" innewohnt (Herzmann \& König, 2015, S. 72)? Selbst durch prominente Vertreter des Expertenansatzes zum Lehrberuf wie David Berliner, die gewiss nicht im Verdacht stehen, etwa von einer Berufung zum Lehren auszugehen, wird die Frage nach der Bedeutung einer persönlichen Eignung, Begabung oder eines Talents für die Anforderungen bestimmter Berufe aufgeworfen: „The question that needs to be answered is how, and in what ways, does talent influence the development of expertise in domains like music, wrestling or teaching?" (Berliner, 2001, S. 464). Eine Begabung oder das Talent zum Unterrichten wird dabei als komplexes Zusammenspiel zahlreicher Eigenschaften wie Umgänglichkeit, Überzeugungskraft, Vertrauenswürdigkeit, die Fähigkeit, mehr als eine Sache gleichzeitig zu tun, usw. beschrieben (vgl. ebd., S. 465). Eine solche Rede von Talenten und Begabungen soll hier sicherlich nicht die zentrale Bedeutung des Erwerbs von Wissen und Fertigkeiten bzw. berufsrelevanten Kompetenzen anzweifeln. Genauso gewiss wird man es aber aller Wahrscheinlichkeit auch immer begrüßen, wenn Personen, die den Lehrberuf ergreifen (möchten), von vornherein ,über gewisse ,menschliche Stärken“" (Reichenbach, 2006, S. 249) im Sinne der genannten Eigenschaften verfügen.

\section{2 „Lehrerpersönlichkeit“ und Lehrerinnenbildung}

In der Diskussion wird nun die Idee der „Lehrerpersönlichkeit“ einerseits der Lehrer*innenbildung entgegengestellt: Denn „[d]ie Lehrerpersönlichkeit kann man nicht lernen" (Schaaf, 2015). Ausgegangen wird bereits in Einlassungen auf die "Psychologie der Lehrerpersönlichkeit" in den 1980er-Jahren (Dietrich, 1983; vgl. aktuell Eckert \& Sieland, 2017; Haag \& Streber, 2020) von der Annahme, dass der „Lehrbarkeit der Erziehung" Grenzen gesetzt seien, „weil akademische Lehre keine grundsätzliche Persönlichkeitsbildung bewirken kann" (Dietrich, 1983, S. 15). Es sei eben ein Unterschied, „ob ein Lehrer sich autoritär verhält oder ob er eine Autorität ist" (ebd., S. 16). Gelernt werden könne, sich nicht autoritär zu verhalten; zu bezweifeln sei aber, ob es auch erlernt werden könne, eine Autorität zu sein (ebd.). Und 
auch wenn aktuell die „Bedeutung der Kompetenzen für den Lehrerberuf“ im kompetenzorientierten Ansatz hervorgehoben wird, so dürfe „nicht übersehen werden, dass kaum alle Voraussetzungen, die ein guter und professionell Lehrender benötigt, erlernbar sind“ (Bohl, Harant \& Wacker, 2015, S. 59).

Andererseits wird im Lehrer*innenbildungsdiskurs die „Lehrerpersönlichkeit“ als Gegenstand und Ziel, Lehrer*innenbildung und Professionalisierung als Persönlichkeitsbildung, als Entwicklung hin zu einer Lehrerpersönlichkeit entworfen bzw. gefordert (u.a. Loebell \& Martzog, 2017; Liebig \& Gängler, 2018; Röbe et al., 2019). Lehrer*innenbildung dürfe sich nicht in der Wissensvermittlung erschöpfen, sondern sie müsse sich die „ganzheitliche Bildung der Lehrerpersönlichkeit zum vollen Menschen als ihre Aufgabe vornehmen" (Wagenreich, 2015, S. 105). Gefordert wird eine "ganzheitliche Ausbildung von Lehrerinnen und Lehrern“ (Arndt, 2009, S. 32). „Persönlichkeits- und Kompetenzbildung“ müssten Hand in Hand gehen, „was eine Perspektiverweiterung der Lehrerbildung erfordert“ (Loebell \& Martzog, 2017, S. 9). Bezogen auf den Lehrberuf und die Lehrer*innenbildung wird im Zusammenhang mit der Rede von der „Lehrerpersönlichkeit“ auch von der Entwicklung einer notwendigen und unterstellt berufstypischen Haltung gesprochen (Röbe et al., 2019, S. 289). „Speziell in den ,Schulpraktischen Studien' dürfte die Chance zur Entwicklung eigenständiger ,LehrerInnenpersönlichkeiten' hoch sein“" (Teml \& Unterweger, 2002, S. 10). Konkret werden für die Persönlichkeitsförderung innerhalb der Lehrer*innenbildung u. a. Selbsterfahrungsgruppen (selbsterfahrungsorientierte Veranstaltungen) vorgeschlagen, und Lehrer*innenbildung wird hier auch in einen therapeutischen Kontext eingebettet (ebd., S. 12f.). ${ }^{1}$ Die Förderung der Persönlichkeitsentwicklung von Lehramtsstudierenden werde jedoch, so die Kritik, generell vernachlässigt; sie werde zugleich zunehmend dringlicher (ebd.).

Mit Blick auf die Persönlichkeitsmerkmale von angehenden Lehrkräften in der Lehrer*innenbildung stehen sich zwei Positionen gegenüber: (1.) die Annahme, dass die Entwicklung der Lehrerpersönlichkeit, wie zuletzt gezeigt, zu den Zielen der Lehrer*innenbildung zählt, wobei hier ein diffuses Verständnis von Lehrerpersönlichkeit vorherrscht. Dem steht (2.) die Annahme einer weitgehenden Stabilität der Persönlichkeitsmerkmale entgegen, die als zum Teil genetisch determinierte individuelle Eingangsvoraussetzungen bzw. personale Ausgangslagen gelten (vgl. Cramer, 2012, S. 216 f.).

1 Mit der hier geforderten konkreten Maßnahme und Verantwortung für die Persönlichkeitsbildung der angehenden Lehrkräfte wird ein konkretes Beispiel für den von Krammer und Pflanzl (2019) begründet befürchteten Übergang von der (akademischen) Lehrerinnenbildung zur Therapie gegeben. 


\section{Exkurs: Persönlichkeitsfaktoren (angehender) Lehrkräfte und Lehrer ${ }^{\star}$ innenbildung(sforschung)}

Allgemeine Persönlichkeitsmerkmale gelten in der Lehrer ${ }^{\star}$ innenbildungsforschung als Bedingung der professionellen Entwicklung angehender Lehrkräfte (Rothland, 2014; Cramer, 2016a, 2016b). Sie werden als „Ensemble relativ stabiler Dispositionen, die für das Handeln, den Erfolg und das Befinden im Lehrerberuf bedeutsam sind“ (Mayr \& Neuweg, 2006, S. 182), charakterisiert. Damit fokussiert die Forschung auf Persönlichkeitsmerkmale als personenbezogene, berufsunspezifische Dispositionen im Anschluss an in der Persönlichkeitspsychologie unterschiedene Persönlichkeitsfaktoren. Eine Grundidee ist hier, dass die Nutzung der Lern- und Qualifikationsangebote, die in den Lerngelegenheiten im Rahmen der Lehrer*innenbildung bereitgestellt werden, durch die personalen Ausgangslagen der angehenden Lehrkräfte bedingt wird (Kunter, Kleickmann, Klusmann \& Richter, 2011). Interindividuelle Unterschiede in der Ausprägung professioneller Handlungskompetenz von Lehrkräften, ihrer Performanz und in der Wirkung ihres Handelns etwa auf die Schülerinnenleistung werden somit auch auf ihre persönlichen Voraussetzungen zurückgeführt, ohne die Bedeutung der Qualifikation im Rahmen der Lehrer*innenbildung sowie der Fort- und Weiterbildung in Abrede zu stellen (Cramer \& Rothland, 2021).

Über die Befunde zur Bedeutung von Persönlichkeitsfacetten für die Nutzung und Wirkung der Lehrerinnenbildung sowie die Qualität der Berufstätigkeit und des Berufserlebens von Lehrkräften hinaus (vgl. Hanfstingl, 2019; Klassen \& Tze, 2014; Mayr, 2014, 2016) verweisen die Forschungsbefunde im Vergleich zu anderen Berufsund Studierendengruppen generell auf kein lehrberufs- bzw. lehramtsspezifisches Persönlichkeitsprofil (Eulenberger, 2015; Rothland, 2014). Vielmehr variieren die Ausprägungen der einzelnen Faktoren von Stichprobe zu Stichprobe zum Teil erheblich, sodass in der Summe kein einheitliches Bild von den Persönlichkeitsmerkmalen angehender Lehrkräfte gezeichnet werden kann. Oder anders formuliert: Die „Lehrerpersönlichkeit“ bzw. das lehramtsspezifische Persönlichkeitsprofil gibt es nicht.

\section{Diskussion: „Lehrerpersönlichkeit“ - das nicht vermittelbare Berufsgeheimnis des Lehrberufs?}

Der hier in Rede stehende Diskurs zur „Lehrerpersönlichkeit“ ist jedoch von der im Exkurs angesprochenen Forschung zur Persönlichkeit (angehender) Lehrer*innen (zur Unterscheidung: Mayr \& Paseka, 2002) entkoppelt: „die Big Five sagen noch nicht, was Lehrerpersönlichkeit ist. Mit anderen Worten, Lehrerpersönlichkeit ist etwas anderes als das Zusammengehen psychologischer Persönlichkeitsmerkmale“ (Schelten, 2009, S. 39). 
Mit dem Konstrukt „Lehrerpersönlichkeit“ verbindet sich auch aktuell, wie skizziert, die Verkörperung von idealen bzw. gesellschaftlich erwünschten Charaktereigenschaften, Einstellungen und Werten in der Person der Lehrkräfte, die diese als Haltungen verinnerlicht haben und (vor-)leben. In bester geisteswissenschaftlicher Tradition werden diese Persönlichkeitsideale in Tugendkatalogen für den Lehrberuf aufgelistet, bspw. in den „Verhaltensdimensionen von humanistischen Lehrerpersönlichkeiten“" nach Dubs (2009, S. 365 ff.), und als Teil der Charakterisierung der "Lehrerpersönlichkeit“ unkritisch angeführt (so bei Haag \& Streber, 2020, S. 110, S. $135 \mathrm{ff}$.).

Zweitens verbindet sich mit der „Lehrerpersönlichkeit“ die Annahme erzieherischer bzw. pädagogischer Wirksamkeit, die als bedeutsamer eingestuft wird als etwa die fachliche oder (fach-)didaktische Expertise. Die „Lehrerpersönlichkeit“ wird schließlich ganzheitlich gedacht. Sie entwickelt sich nicht schrittweise bzw. wird über einen längeren Zeitraum erworben, sondern sie ist entweder von vornherein als Ganzes gegeben oder fällt einem ggf. als Ganzes zu (Bürkler, Rosenmund \& Schmid, 2005, S. 103). Darüber hinaus impliziert der Begriff der „Lehrerpersönlichkeit“ im Übrigen auch, dass die gesamte Person und Lebensführung dem Beruf zu- bzw. untergeordnet wird (Pfützner, 2013). Die Trennung von „Leben“ und Beruf erscheint so gar nicht möglich. Eine „Lehrerpersönlichkeit“ ist man nicht nur in der Schule!

In jedem Falle erscheint die "Lehrerpersönlichkeit“ in der Diskussion als etwas Diffuses, nicht Mess- oder Operationalisierbares. Dies wird jedoch weniger als Problem, sondern in erster Linie als Besonderheit ausgewiesen. Und obwohl sie nicht recht bestimmbar erscheint - bei Haag und Streber (2020, S. 165 f.) werden ihr in der Summe 92 Attribute zugeordnet (Doppelungen inbegriffen) und so die Beliebigkeit der Begriffsbestimmung auf ein bisher unerreichtes Niveau gebracht -, wird die „Lehrerpersönlichkeit“ als der entscheidende Faktor nicht nur von berufserfahrenen Lehrkräften benannt (Herrmann \& Hertramph, 2002), als der Faktor, der den Unterschied macht:

„Es ist eben nicht dasselbe, wenn zwei Lehrer das Gleiche tun: der eine wirkt lächerlich, wenn er einen Wutanfall produziert, der andere schüchtert die Kinder ein. Unterschiedliche Effekte gleicher Verhaltensäußerungen müssen zwangsläufig durch den Persönlichkeitshintergrund erklärt werden“" (Dietrich, 1983, S. 15).

Es gebe keinen Zweifel daran, „dass es Personen gibt, die auf Grund ihrer Persönlichkeit [...] besser oder positiver auf Kinder wirken als andere" (Fink-Pomberger \& Volkmer, 2015, S. 9). „Der Grund, warum manche Lehrpersonen eine besonders gute Beziehung zu ihren Schülern und Schülerinnen eingehen können, liegt zweifellos in ihrer Persönlichkeit" (Kommenda, 2015, S. 144). Schließlich kämen Wissenschaft und Praxis bzw. die Praktiker*innen gleichermaßen zunehmend zu der Erkenntnis, „dass guter Unterricht nicht das Resultat fachwissenschaftlicher Expertise“ ist, sondern die 
„Persönlichkeit des Lehrers“ ausschlaggebend für den Lernerfolg der Schüler*innen sei (Loebell \& Martzog, 2017, S. 9, mit Verweis auf die Hattie-Studie).

Das skizzierte Festhalten am Konstrukt der „Lehrerpersönlichkeit“ wird auch in der Gegenwart zusammengefasst u.a. damit begründet, dass mittels empirischer Forschung erfolgreiches Lehrer*innenhandeln nicht hinreichend erklärt werden könne. "Lehrerpersönlichkeit“ selbst lasse sich auch nicht messen (Schelten, 2009, S. 39): „Offensichtlich gibt es in der Wirkung einer Lehrerin oder eines Lehrers neben dem Bestimmbaren auch ein Unbestimmbares“ (ebd., S. 40). Und das, was nicht bestimmt, was nicht gemessen werden kann, das ist - so die Argumentation - die für das erfolgreiche pädagogische Handeln entscheidende besondere Ausprägung einer „Lehrerpersönlichkeit“. Sie kann als Kompensation eines fehlenden Berufsgeheimnisses im Lehrberuf (vgl. Rothland, 2013) bzw. als Anspruch auf ein doch vorhandenes Berufsgeheimnis angesehen werden. Wenn berufserfahrene Lehrkräfte selbst den Faktor „Lehrerpersönlichkeit“ betonen, so immunisieren sie sich zudem auch gegen die Kontrolle ihres beruflichen Handelns, indem sie die Wirksamkeit ihres Tuns an ihre persönlichen Eigenschaften und nicht an spezifische Kompetenzen knüpfen (Bürkler, Rosenmund \& Schmid, 2005, S. 104), verbunden mit dem Anspruch auf Exklusivität, dass nicht jede*r Lehrer ${ }^{\star}$ in werden könne.

Was aber nicht $\mathrm{zu}$ fassen, nicht $\mathrm{zu}$ bestimmen ist, was nicht identifizier- und (be-) greifbar erscheint, das kann weder erlernt noch vermittelt werden. Insofern erscheint auch das Ansinnen, Professionalität im Lehrberuf an die Bildung der „Lehrerpersönlichkeit“ zu knüpfen, konsequent gedacht unmöglich. Wie soll etwas gelernt oder ausgebildet werden, von dem nicht genau gesagt werden kann, was es ist? Die erfolgreiche Berufstätigkeit von Lehrer*innen wird, so die Bilanz, in diesem nach wie vor auch im wissenschaftlichen Lehrer ${ }^{\star}$ innenbildungskontext populären Diskurs letztlich eher im Reich des Geheimnisvollen oder gar des Magischen denn im rationalen Kontext von Profession, Professionalität und berufspraktischem Handeln verortet. Man könne „den Lehrerberuf auf Dauer nur dann positiv gestalten, wenn man als Pädagoge in der Lage ist, seine Schüler - einem Magier gleich - immer wieder zu verzaubern“ (Maier, 2018, S. 142).

2 So wird bspw. auch in der Einleitung zur Buchpublikation von Haag und Streber (2020, S. 7) von einem "klassischen Beispiel“ ausgegangen, das als „Topos der Lehrerpersönlichkeit“ anzusehen sei: Obwohl eine Lehrkraft nur ein bescheidenes, wenig aktivierendes Methodenrepertoire einsetze, würden die Schüler*innen an deren Lippen hängen und lernen. Ein solches Beispiel lasse sich „wissenschaftlich wenig greifen“, daher werde hier „verlegen auf die Persönlichkeit verwiesen“. 


\section{Literatur und Internetquellen}

Arndt, E. (2009). „Arbeiten Sie an Ihrer LP!“ - Bedeutung und Entwicklung der Lehrerpersönlichkeit. Themenzentrierte Interaktion, 23 (2), 32-40.

Berliner, D. C. (2001). Learning About and Learning from Expert Teachers. International Journal of Educational Research, 35 (5), 463-482. https://doi.org/10.1016/S08830355(02)00004-6

Bohl, T., Harant, M., \& Wacker, A. (2015). Schulpädagogik und Schultheorie. Bad Heilbrunn: Klinkhardt/UTB.

Bromme, R., \& Haag, L. (2008). Forschung zur Lehrerpersönlichkeit. In W. Helsper \& J. Böhme (Hrsg.), Handbuch der Schulforschung (2., erw. Auf.) (S. 803-819). Wiesbaden: VS. https://doi.org/10.1007/978-3-531-91095-6_32

Bürkler, S., Rosenmund, M., \& Schmid, C. (2005). Die „Lehrerpersönlichkeit“. Einige Thesen zur Diskussion. Zeitschrift für pädagogische Historiographie, 11 (2), 103-105.

Cramer, C. (2012). Entwicklung von Professionalität in der Lehrerbildung. Empirische Befunde zu Eingangsbedingungen, Prozessmerkmalen und Ausbildungserfahrungen Lehramtsstudierender. Bad Heilbrunn: Klinkhardt.

Cramer, C. (2016a). Personale Merkmale Lehramtsstudierender als Ausgangslage der professionellen Entwicklung. Dimensionen, Befunde und deren Implikationen für die Lehrerbildung. In A. Boeger (Hrsg.), Eignung für den Lehrerberuf: Auswahl und Förderung (S. 31-56). Wiesbaden: Springer VS. https://doi.org/10.1007/978-3-65810041-4_3

Cramer, C. (2016b). Berufswahl Lehramt: Wer entscheidet sich warum? In M. Rothland (Hrsg.), Beruf Lehrer/Lehrerin. Ein Studienbuch (S. 261-276). Münster: Waxmann.

Cramer, C., \& Rothland, M. (2021). Pädagogische Professionelle in der Schule. In T. Hascher, W. Helsper \& T.-S. Idel (Hrsg.), Handbuch Schulforschung (3. Aufl.). Wiesbaden: Springer VS. https://doi.org/10.1007/978-3-658-24734-8_56-1

Dietrich, R. (1983). Einführung und Übersicht. In R. Dietrich, E. Elbing, I. Peagitsch \& H. Ritscher (1983), Psychologie der Lehrerpersönlichkeit (S. 11-23). München \& Basel: Ernst Reinhardt.

Draxler, B. (2015). Lehrerpersönlichkeit - was ist das? In E. Rauscher (Hrsg.), Von der Lehrperson zur Lehrerpersönlichkeit (S. 97-100). Innsbruck: StudienVerlag.

Dubs, R. (2008). Lehrerbildung zwischen Theorie und Praxis. In E.-M. Lankes (Hrsg.), Pädagogische Professionalität als Gegenstand empirischer Forschung (S. 11-28). Münster: Waxmann.

Dubs, R. (2009). Lehrerverhalten. Ein Beitrag zur Interaktion von Lehrenden und Lernenden im Unterricht. Stuttgart: Steiner.

Eckert M., \& Sieland, B. (2017). Psychologie der Lehrerpersönlichkeit. In M. Schweer (Hrsg.), Lehrer-Schüler-Interaktion (3. Aufl.) (S. 147-165). Wiesbaden: Springer VS. https://doi.org/10.1007/978-3-658-15083-9_6

Eulenberger, J. (2015). Die Persönlichkeitsmerkmale von Personen im Kontext des Lehrer_innenberufs (SOEPpaper on Multidisciplinary Panel Data Research, 788). Berlin: DIW. https://doi.org/10.2139/ssrn.2666931

Fink-Pomberger, S., \& Volkmer, I. (2015). Lehrerinnen- und Lehrerpersönlichkeit. Bildung braucht Persönlichkeiten mit Kopf, Herz und Humor. Hamburg: Dr. Kovač.

Getzels, J. W., \& Jackson, P. W. (1970). Merkmale der Lehrerpersönlichkeit. In K. H. Ingenkamp \& E. Parley (Hrsg.), Handbuch der Unterrichtsforschung, Bd. 2 (S. 13521526). Weinheim, Basel: Beltz.

Gudjons, H. (2013). Didaktik zum Anfassen. Lehrer/in-Persönlichkeit und lebendiger Unterricht (3., durchges. Aufl.). Bad Heilbrunn: Klinkhardt. 
Haag, L. (2013). Die Lehrerpersönlichkeit als Erziehungsfaktor. In L. Haag, S. Rahm, H. J. Apel \& W. Sacher (Hrsg.), Studienbuch Schulpädagogik (5., vollst. überarb. Aufl.) (S. 366-387). Bad Heilbrunn: Klinkhardt/UTB.

Haag, L., \& Streber, D. (2020). Lehrerpersönlichkeit. Die Frage nach dem, guten Lehrer, nach der, guten Lehrerin'. Bad Heilbrunn: Klinkhardt

Hanfstingl, B. (2019). Lehrerpersönlichkeit: kritische Würdigung und Ausblick. In U. Steffens \& P. Posch (Hrsg.), Lehrerprofessionalität und Schulqualität (S. 97-110). Münster \& New York: Waxmann.

Herrmann, U. (2000). Der lange Abschied vom „geborenen Erzieher“. Lehrerpersönlichkeit, Lehrerausbildung, Lehrerberuf und -berufsalltag - Erwartungen, Positionen und Thesen vom Ende der 40er bis zu den 70er Jahren. In J. Bastian, W. Helsper, S. Reh \& C. Schelle, (Hrsg.), Professionalisierung im Lehrerberuf (S. 15-32). Opladen: Leske + Budrich.

Herrmann, U., \& Hertramph, H. (2002). „Lehrer“ - eine Selbstdefinition. Ein Ansatz zur Analyse von "Lehrerpersönlichkeit“ und Kompetenzgenese durch das sozial-kognitive Modell der Selbstwirksamkeitsüberzeugung. In U. Herrmann (Hrsg.), Wie lernen Lehrer ihren Beruf? Empirische Befunde und praktische Vorschläge (S. 200-220). Weinheim \& Basel: Beltz.

Herzmann, P., \& König, J. (2015). Lehrerberuf und Lehrerbildung. Bad Heilbrunn: Klinkhardt/UTB.

Klassen, R. M., \& Tze, V. M. (2014). Teachers' Self-Efficacy, Personality, and Teaching Effectiveness: A Meta-Analysis. Educational Research Review, 12, 59-76. https://doi. org/10.1016/j.edurev.2014.06.001

Kommenda, D. (2015). Persönlichkeitsbildung in der Pädagogik. Auf der Suche nach dem Idealbild der Lehrerpersönlichkeit. In E. Rauscher (Hrsg.), Von der Lehrperson zur Lehrerpersönlichkeit (S. 139-144). Innsbruck: StudienVerlag.

Krammer, G., \& Pflanzl, B. (2019). Können wir jede Person lehren Lehrer*in zu werden? Sollen wir es? journal für lehrerInnenbildung, 19 (2), 28-39. https://doi.org/10.35468/ jlb-02-2019_02

Kunter, M., Kleickmann, T., Klusmann, U., \& Richter, D. (2011). Die Entwicklung professioneller Kompetenz von Lehrkräften. In M. Kunter, J. Baumert, W. Blum, U. Klusmann, S. Krauss \& M. Neubrand (Hrsg.), Professionelle Kompetenz von Lehrkräften. Ergebnisse des Forschungsprogramms COACTIV (S. 55-68). Münster et al.: Waxmann.

Liebig, M., \& Gängler, H. (2018). Lehrerinnen- und Lehrerbildung als Persönlichkeitsbildung. Berufs- und Wirtschaftspädagogik - online, 34, 1-14.

Loebell, P., \& Martzog, P. (2017). Einleitung. In P. Loebell \& P. Martzog (Hrsg.), Wege zur Lehrerpersönlichkeit. Kompetenzerwerb, Persönlichkeitsentwicklung und aktuelle Herausforderungen in der Lehrerbildung (S. 9-12). Opladen et al.: Barbara Budrich. https://doi. org/10.2307/j.ctvddztqd.4

Maier, P. (2018). Archetypen der Lehrerpersönlichkeit. Ein supervisorisches Modell für die verschiedenen Rollen der Lehrkraft. Schulverwaltung Baden-Württemberg, 27 (5), 141143.

Mayr, J. (2014). Der Persönlichkeitsansatz in der Forschung zum Lehrerberuf. Konzepte, Befunde und Folgerungen. In E. Terhart, H. Bennewitz \& M. Rothland (Hrsg.), Handbuch der Forschung zum Lehrerberuf (2., überarb. u. erw. Aufl.) (S. 189-215). Münster et al.: Waxmann.

Mayr, J. (2016). Lehrerpersönlichkeit. In M. Rothland (Hrsg.), Beruf Lehrer/Lehrerin. Ein Studienbuch (S. 87-102). Münster: Waxmann.

Mayr, J., \& Paseka, A. (2002). „Lehrerpersönlichkeit“. journal für lehrerInnenbildung, 2 (2), 50-55.

Messner, R. (2001). Szenarien zur Bearbeitung des Theorie-Praxis-Problems in der Lehrerbildung. journal für lehrerInnenbildung, 1 (2), 10-19. 
Neuweg, H. G. (2008). Grundlagen der Lehrer/innen/kompetenz. Odgojne znanosti, 10 (1), $13-22$.

Paffrath, H. (1980). Die Lehrerpersönlichkeit - Über Faszination und Krise eines Begriffs. In KEG Katholische Erziehergemeinschaft in Bayern e.V. (Hrsg.), Die Bedeutung der Lehrerpersönlichkeit für Erziehung und Unterricht (S. 11-28). Donauwörth: Auer.

Pfützner, R. (2013). Beherrschte Herrscher_innen. Fragmentarische Anmerkungen zum Begriff der Lehrerpersönlichkeit. In K. Kenklies (Hrsg.), Person und Pädagogik. Systematische und historische Zugänge zu einem Problemfeld (S. 43-55). Bad Heilbrunn: Klinkhardt.

Reichenbach, R. (2006). Schulmeisterliche Lehrerprofession: Bemerkungen zur Beschreibung und Identität von Lehrpersonen. In P. Hanke (Hrsg.), Grundschule in Entwicklung. Herausforderungen und Perspektiven für die Grundschule heute (S. 249-261). Münster et al.: Waxmann.

Röbe, E., Aicher-Jakob, M., \& Seifert, A. (2019). Lehrer werden, Lehrer sein, Lehrer bleiben. Lehrerberuf zwischen Schulalltag und Professionalisierung. Paderborn et al.: Schöningh/ UTB.

Rothland, M. (2013). Beruf Lehrer/Lehrerin - Arbeitsplatz Schule. Charakteristika der Arbeitstätigkeit und Bedingungen der Berufssituation. In M. Rothland (Hrsg.), Belastung und Beanspruchung im Lehrerberuf. Modelle, Befunde, Interventionen (2. Aufl.) (S. 21-39). Wiesbaden: Springer VS. https://doi.org/10.1007/978-3-531-18990-1_2

Rothland, M. (2014). Wer entscheidet sich für den Lehrerberuf? Herkunfts-, Persönlichkeitsund Leistungsmerkmale von Lehramtsstudierenden. In E. Terhart, H. Bennewitz \& M. Rothland (Hrsg.), Handbuch der Forschung zum Lehrerberuf (2. Aufl.) (S. 349-385). Münster: Waxmann.

Rothland, M., Cramer, C., \& Terhart, E. (2018). Forschung zum Lehrerberuf und zur Lehrerbildung. In R. Tippelt \& B. Schmidt-Hertha (Hrsg.), Handbuch Bildungsforschung (4. Aufl.) (S. 1011-1034). Wiesbaden: Springer VS. https://doi.org/10.1007/978-3-53119981-8_44

Schaaf, J. (2015). „Die Lehrerpersönlichkeit kann man nicht lernen“. Frankfurter Allgemeine Sonntagszeitung vom 30.08.2015.

Schelten, A. (2009). Lehrerpersönlichkeit - ein schwer fassbarer Begriff. Die berufsbildende Schule, 61 (2), 39-40.

Teml, H., \& Unterweger, E. (2002). Persönlichkeitsförderung in der LehrerInnenbildung. journal für lehrerInnenbildung, 2 (2), 7-21.

Wagenreich, U. (2015). Lehrerpersönlichkeit - was ist das? Der Weg von einer frisch graduierten Lehrperson zur Lehrerpersönlichkeit. In E. Rauscher (Hrsg.), Von der Lehrperson zur Lehrerpersönlichkeit (S. 101-107). Innsbruck: StudienVerlag.

Weiß, S., Schramm, S., \& Kiel, E. (2013). Wie sollen Grundschullehrkräfte sein? Die Sicht von Lehrkräften und in der Lehrerausbildung tätigen Personen auf die Lehrerpersönlichkeit. Pädagogische Rundschau, 67 (4), 455-464.

Werner, R. (2013). Lehrerrolle und Lehrerpersönlichkeit. Katholische Bildung, 114 (2), 4957.

Martin Rothland, Prof. Dr., geb. 1974, Professor für Erziehungswissenschaft mit Schwerpunkt Allgemeine Didaktik und Unterrichtsforschung.

E-Mail: Martin.Rothland@uni-muenster.de

Korrespondenzadresse: Westfälische Wilhelms-Universität Münster, Fachbereich 06, Institut für Erziehungswissenschaft, Bispinghof 5/6, 48143 Münster 
Anje Ostermann, Anke Lindmeier, Hendrik Härtig, Lorenz Kampschulte, Mathias Ropohl \& Julia Schwanewedel

\title{
Mathematikspezifische Medien nutzen: Was macht den Unterschied - Lehrkraft, Schulkultur oder Technik?
}

\section{Zusammenfassung}

Forschung zu Mediennutzung im Unterricht nimmt bisher selten einen fachlichen Blick ein, obwohl gerade im Mathematikunterricht spezifische Medien im Einsatz sind. Die berichtete Lehrkräftebefragung zeigt die aktuelle mathematikspezifische Mediennutzung in der Sekundarstufe auf und erklärt Unterschiede durch individuelle und organisationale Faktoren im Kontrast unterschiedlicher Gruppen von Nutzungshäufigkeit. Weiter diskutieren wir Implikationen zur Förderung von Medieneinsatz im Mathematikunterricht. Schlüsselwörter: Mathematikunterricht, Medieneinsatz, Lehrkräfte, Schulkultur

\section{Using Mathematics-specific Media: What Makes the Difference - Teachers, School-Culture, or Equipment?}

\begin{abstract}
Research on media use in teaching has as yet rarely taken a subject-specific view, despite the fact that specific tools are used in mathematics instruction in particular. The reported teacher survey shows the current mathematics-specific media use in secondary level education in Germany and explains differences by individual and organizational factors within different groups of usage frequency. Furthermore, we discuss implications for promoting media use in mathematics instruction.

Keywords: mathematics education, media use, teachers, school-culture
\end{abstract}

\section{$1 \quad$ Einleitung}

In der Schule wächst die Rolle digitaler Medien (KMK, 2016), wobei die Digitalisierung als schulische Querschnittsaufgabe gesehen wird (van Ackeren et al., 2019). Untersuchungen zur Mediennutzung folgen entsprechend meist einem fachübergrei- 
fenden Blick, etwa werden Merkmale wie die Ausstattung an der Schule oder deren Nutzung ohne Fachunterscheidung beschrieben (z.B. Eickelmann et al., 2019; BITKOM, 2015). Gleichwohl ist Schule überwiegend in Unterrichtsfächern strukturiert, was zur Folge hat, dass auch eine Querschnittsaufgabe letztlich im Fachunterricht realisiert werden muss. Nur wenige Studien nehmen bisher jedoch eine spezifischere Perspektive ein und untersuchen, wie Medien im (MINT-)Fachunterricht integriert werden (z.B. Hillmayr, Reinhold, Ziernwald \& Reiss, 2017; Lorenz, Bos \& Endberg, 2017).

Dies ist umso verwunderlicher, als aus fachlicher Sicht der sinnvolle Einsatz spezifischer Werkzeuge als wichtig erachtet wird (Härtig, Kampschulte, Lindmeier, Ostermann, Ropohl \& Schwanewedel, 2018). Im Fach Mathematik sollen z. B. dynamische Geometrie-Systeme (DGS, ermöglichen die Dynamisierung von Konstruktionen), Tabellenkalkulationsprogramme (TKP, ermöglichen die Verarbeitung numerischer Daten in Tabellenform) oder Computer-Algebra-Systeme (CAS, ermöglichen die exakte Umformung algebraischer Ausdrücke), eingesetzt und der Umgang mit ihnen geübt werden (KMK, 2015). Die Nutzung dieser Medien ist vermutlich, ebenso wie die Mediennutzung im Allgemeinen, von Merkmalen auf Ebene der Lehrkräfte (z. B. Selbstwirksamkeitserwartung, Einstellungen) und Ebene der Schule (z. B. Ausstattung, schulinterne Regelungen) abhängig (Prasse, 2012). Es ist aber anzunehmen, dass Merkmale auf Ebene der Schul-Fachkultur ebenso eine Rolle spielen (Barzel, 2012). Bisher gibt es dazu jedoch kaum Untersuchungen und so bleibt es ungewiss, ob die fachunabhängig gewonnenen Erkenntnisse zur Mediennutzung, sowie deren Bedingungsfaktoren auf organisationaler Ebene und Ebene der Lehrkräfte auch für fachspezifische Medien gelten.

Daher ist ein Ziel der hier vorgestellten Studie, unter einem stärker fachspezifischen Blick zu erfassen, wie sich der Einsatz mathematikspezifischer Medien im Unterricht am Beispiel von CAS und DGS darstellt. Darüber hinaus sollen Einflüsse der organisationalen Rahmenbedingungen und der Merkmale von Lehrpersonen auf den fachspezifischen Medieneinsatz untersucht werden, um geeignete Ansatzpunkte für die Lehrkräfteaus- und -weiterbildung im Bereich des Medieneinsatzes im Mathematikunterricht $\mathrm{zu}$ identifizieren.

\section{Theoretischer Hintergrund}

Mathematikunterricht mit digitalen Medien ist Gegenstand vieler Untersuchungen: Häufig steht dabei die Frage danach, wie Schüler*innen mathematische Inhalte mithilfe digitaler Medien lernen, im Fokus (u.a. Hoffkamp, 2011; Rieß, 2018; Weigand \& Bichler, 2010). So untersuchen Rolfes und Kollegen (2020) bspw. die Wirkung verschiedener Visualisierungen mathematischer Inhalte (statisch, dynamisch und dyna- 
misch mit Interaktionsmöglichkeit) auf das Lernen im Bereich Funktionen und finden dabei einen Vorteil dynamischer Visualisierungen.

Andere Arbeiten bearbeiten die Frage nach der Gestaltung digitaler Medien im Mathematikunterricht und damit einhergehend der Strukturierung und den Realisierungen von Lerninhalten mithilfe digitaler Medien. So finden sich einige Untersuchungen, die das Design mediengestützter Aufgaben oder umfassenderer mathematischer Lernumgebungen in den Blick nehmen (u. a. Fahlgren \& Brunström, 2014; Venturini \& Sinclair, 2017).

Erkenntnisse solcher Untersuchungen tragen dazu bei, eine wirkungsvolle Gestaltung und Integration digitaler Medien im Mathematikunterricht $\mathrm{zu}$ verstehen und zu fördern. Ob digitale Medien in den Mathematikunterricht integriert werden und welche Faktoren dafür relevant sind, lässt sich daraus jedoch nur bedingt ableiten. Allerdings können verschiedene allgemeine Ansätze zur Beschreibung des Technologieeinsatzes in der Schule Hinweise auf Antworten zu diesen Fragen liefern. Prasse (2012) arbeitet heraus, dass diese Ansätze - wenn auch in unterschiedlicher Feinkörnigkeit - ähnliche Einflussfaktoren annehmen, die auf mehreren Ebenen wirken. Dabei lässt sich ein Teil der Einflussfaktoren für die Nutzung von Technologien auf der individuellen Ebene der Lehrkraft verorten. Andere können als organisationale Merkmale der Schule charakterisiert werden.

Auf der individuellen Ebene findet sich allgemein ein positiver Zusammenhang zwischen der Nutzungshäufigkeit von Informations- und Kommunikations-Technologien (IKT), wie z.B. Computer, im Unterricht und den Einstellungen von Lehrkräften (u. a. Hermans, Tondeur, van Braak \& Valcke, 2008; Petko, 2012; Prasse, 2012). Auch die Selbstwirksamkeitserwartung der Lehrkräfte in Bezug auf das Unterrichten mit IKT scheint einen positiven Effekt auf die Nutzungshäufigkeiten von IKT im Unterricht zu haben (u.a. Drossel, Eickelmann \& Gerick, 2017; Petko, 2012). Dieser Zusammenhang konnte auch für den Mathematikunterricht international (u.a. Thomas \& Palmer, 2014; Handal, Cavanagh, Wood \& Petocz, 2011) und im deutschen Kontext (Thurm, 2020) gezeigt werden.

Auf der organisationalen Ebene der Schule gibt es Hinweise, dass die quantitative Ausstattung mit IKT einen positiven Effekt auf die Nutzungshäufigkeit im Unterricht hat (Petko, 2012). Eickelmann, Gerick und Koop (2017) finden diesen Zusammenhang auch speziell für den Mathematikunterricht. Petko (2012) führt jedoch an, dass die reine Anzahl verfügbarer Computer nur bedingt aussagekräftig zur Beurteilung der Ausstattung ist. Ob die Geräte problemlos zugänglich im Klassenraum sind oder nur in Computerräumen zur Verfügung stehen, kann z.B. einen Unterschied machen. Prasse (2012), die in die Beurteilung der Ausstattung neben der Quantität auch qualitative Aspekte der Organisation (z. B. unkomplizierte Zugangsregeln, keine zusätzlichen Absprachen notwendig) einbindet, findet jedoch 
keinen Zusammenhang der Ausstattung mit der Nutzungshäufigkeit im Unterricht. Ein einheitliches Bild zeigt sich an dieser Stelle also nicht. Zuletzt zeigen schulinterne Regelungen wie z.B. eine IKT-Strategie positive Effekte auf die Nutzung von IKT im Unterricht (Prasse, 2012; Tondeur, van Keer, van Braak \& Valcke, 2008).

Eine Untersuchung, die die Bedeutung individueller und organisationaler Merkmale für IKT-Nutzung gleichzeitig berücksichtigte, wurde zu Beginn der 2000er Jahre durchgeführt (Prasse, 2012). Sie konnte zeigen, dass neben den persönlichen Lehrkraftmerkmalen (wie IKT-bezogene Einstellungen, wahrgenommene Kompetenzen) auch organisationale Merkmale (wie gemeinsame Ziele und Visionen an einer Schule, Unterstützung durch die Schulleitung) einen positiven Effekt auf die Nutzung von IKT im Unterricht haben. Individuelle und organisationale Faktoren hängen also in einem komplexen Wechselspiel mit der Nutzungshäufigkeit zusammen.

\section{Forschungsfragen}

Bisherige Studien legen nahe, dass individuelle Lehrkraftmerkmale (wie Kompetenz, Einstellungen und Werthaltungen in Bezug auf Medien) und organisationale Merkmale (wie Zugang oder Schulklima) einen Effekt auf den Einsatz von Medien im Unterricht haben. Empirische Befunde dazu, ob sich dies für die Nutzung fachspezifischer Medien ebenso darstellt wie für die Nutzung von IKT aus genereller Sicht, sind bisher jedoch rar. Besonders für das Fach Mathematik gibt es keine verlässlichen Daten zur tatsächlichen Nutzung oder Analysen zu deren Bedingungsfaktoren. Deswegen können kaum spezifische evidenzbasierte Ansatzpunkte für die Unterstützung der Integration mathematikspezifischer digitaler Medien in den Unterricht abgeleitet werden.

Vor diesem Hintergrund wird in dieser Studie zunächst mithilfe einer Lehrkräftebefragung erhoben, wie sich das aktuelle Nutzungsverhalten am Beispiel von CAS und DGS als mathematikspezifische Medien mit unterschiedlichem Verpflichtungsgrad (DGS: verpflichtend, CAS: teils verpflichtend) im Mathematikunterricht der Sekundarstufe I darstellt (Forschungsfrage 1). Aufbauend darauf soll untersucht werden, inwiefern Personenmerkmale und/oder organisationale Merkmale Unterschiede im Nutzungsverhalten in Bezug auf CAS und DGS erklären können (Forschungsfrage 2). 


\section{Methode}

\subsection{Stichprobe}

Insgesamt wurden $N=163$ Mathematiklehrkräfte, die in der Sekundarstufe I unterrichten, zum Medieneinsatz im Mathematikunterricht befragt (Zeitraum April 2017 - September 2018, wahlweise papierbasiert oder online). Die Lehrkräfte wurden auf Lehrkräftetagungen und über Schulen direkt kontaktiert. Der Großteil der Befragten stammt aus Nordrhein-Westfalen (47\%) und Schleswig-Holstein (33\%). In diesen Bundesländern sind DGS im Unterricht der Sekundarstufe I verpflichtend, die Nutzung von CAS ist optional (Schleswig-Holstein) bzw. nicht in den Lehrplänen aufgeführt (Nordrhein-Westfalen). Daher ist davon auszugehen, dass CAS deutlich seltener genutzt werden als DGS. Die Mehrheit der Lehrkräfte unterrichtet an einem Gymnasium (61 \%). In Anlehnung an Phasenmodelle der Entwicklung professioneller Kompetenz (z.B. Huberman, 1999) wurde die Berufserfahrung zusammengefasst in fünf Phasen erhoben (vgl. Abb. 1).

Abb. 1: Berufserfahrung der Stichprobe $(N=163)$

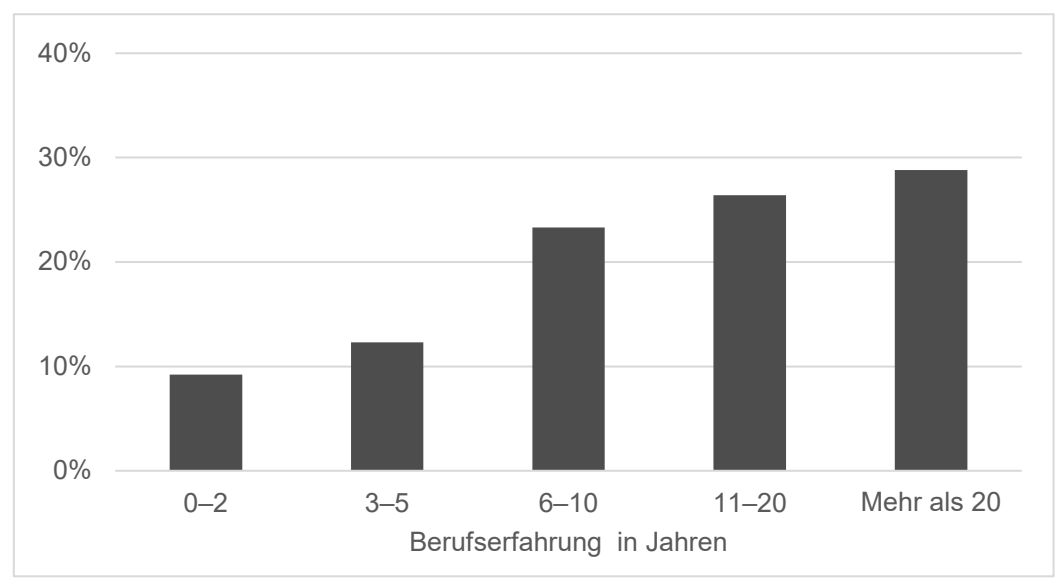

Quelle: eigene Darstellung

Die Gelegenheitsstichprobe wurde mit der Stichprobe einer repräsentativen Untersuchung von Lehrkräften aller Fächer mithilfe zweier Indikatoren aus der BITKOMStudie von 2014 (BITKOM, 2015) verglichen, um Verzerrungen abzuschätzen: In Bezug auf das Verhältnis zu digitalen Medien generell (4-stufiges Antwortformat: 1 = „Ich kann mit digitalen Medien nichts anfangen“, 4 = „Ich bin ein Technik-Fan“) zeigen sich die Mathematiklehrkräfte aus der vorliegenden Studie im Schnitt positiver als die BITKOM-Lehrkräfte $(\Delta=0.13, \mathrm{t}(280.27)=2.69, p=0.008, d=0.32)$. Dem Einsatz digitaler Medien im Unterricht gegenüber (4-stufig: $1=$ „negativ“, $4=$,positiv") zeigen sich die Mathematiklehrkräfte jedoch skeptischer $(\Delta=-0.36$, 
$\mathrm{t}(660)=-6.48, p<0.001, d=-0.50)$. Die Unterschiede sind als kleiner bzw. mittlerer Effekt zu charakterisieren und statistisch signifikant. Die gegensätzlichen Richtungen der Abweichungen lassen keinen Schluss auf Repräsentativität unserer Stichprobe zu, weisen aber auch nicht auf eine systematische positive Verzerrung hin.

\subsection{Instrumente}

In der Befragung wurden individuelle Merkmale auf Ebene der Lehrkräfte, organisationale Merkmale auf Ebene der Schulen sowie die Nutzung der mathematikspezifischen Medien durch die Lehrkräfte erhoben. Dazu konnten teils bestehende Instrumente genutzt oder angepasst werden (vgl. Tab. 1).

Tab. 1: Übersicht erhobener Merkmale und eingesetzter Instrumente

\begin{tabular}{lll}
\hline & Merkmal & Instrument \\
\hline $\begin{array}{l}\text { Individualebene } \\
\text { Lehrkräfte }\end{array}$ & $\begin{array}{l}\text { Einstellungen } \\
\text { zum Einsatz digitaler Medien im Unterricht }\end{array}$ & $\begin{array}{l}\text { Lindau, Kübler \& } \\
\text { Spada, 2013 }\end{array}$ \\
\cline { 2 - 3 } & $\begin{array}{l}\text { Selbstwirksamkeitserwartung } \\
\text { in Bezug auf die Planung und Durchführung von } \\
\text { Unterricht mit digitalen Medien }\end{array}$ & $\begin{array}{l}\text { Adaption nach } \\
\text { Meinhardt, Rabe } \\
\text { \& Krey, 2016 }\end{array}$ \\
\hline $\begin{array}{l}\text { Organisationale } \\
\text { Ebene }\end{array}$ & $\begin{array}{l}\text { Zugang } \\
\text { zu CAS/DGS }\end{array}$ & Eigenentwicklung \\
\cline { 2 - 3 } & $\begin{array}{l}\text { Schulinterne Rege/ungen } \\
\text { bzgl. genutzter CAS-/DGS-Geräte }\end{array}$ & Eigenentwicklung \\
\hline Nutzung & $\begin{array}{l}\text { Nutzungshäufigkeit } \\
\text { bestimmter CAS-/DGS-Lernaktivitäten durch die }\end{array}$ & Eigenentwicklung \\
& $\begin{array}{l}\text { Schüler*innen } \\
\text { (bei typischen Themen) }\end{array}$ & \\
\hline
\end{tabular}

Quelle: eigene Darstellung

Die Einstellungen zum Einsatz digitaler Medien im Unterricht wurden mithilfe einer sechs Items umfassenden Skala erhoben (z. B. „Digitale Medien sorgen im Unterricht für Unruhe und lenken vom Lerninhalt ab“, 5-stufiges Antwortformat: $1=$ „stimme überhaupt nicht zu“, $5=$ „stimme voll zu“, Lindau, Kübler \& Spada, 2013). Die Selbstwirksamkeitserwartungen in Bezug auf die Planung und Durchführung von Unterricht mit digitalen Medien wurde mit einer aus sechs Items bestehenden Skala erfasst. Die Skala der Selbstwirksamkeitserwartung wurde adaptiert nach der physikspezifischen Lehrkräfteselbstwirksamkeitserwartung nach Meinhardt, Rabe und Krey (2016), wobei der Begriff der Selbstwirksamkeitserwartung angelehnt an Schmitz und Schwarzer (2000) als eine Handlung, die mit einer Schwierigkeit verbunden ist, spezifiziert wurde (z.B. „Ich kann den fachlichen Lernprozess durch den Einsatz digitaler Medien unterstützen, auch wenn unvorhergesehene Verständnisschwierigkeiten auftreten“; 4-stufiges Antwortformat: $1=$ „stimmt nicht“, $4=$ „stimmt genau“). Die 
Skalen Einstellungen und Selbstwirksamkeitserwartung beziehen sich auf digitale Medien allgemein und wurden entsprechend nicht spezifisch für CAS und DGS erfasst. Die Skala „Selbstwirksamkeitserwartung beim Unterrichten mit digitalen Medien" befindet sich im Anhang.

Der Zugang zu CAS und DGS als ein Merkmal schulischer Rahmenbedingungen wurde mithilfe je einer Frage erhoben. Es musste aus fünf typischen Optionen diejenige gewählt werden, die die Situation an der eigenen Schule am besten spiegelt (z. B. „DGS sind für alle Lernenden bei Bedarf als Klassensatz verfügbar", vgl. Abb. 2 auf S. 207). Von Lehrkräften ohne Zugang wurden keine weiteren Angaben zu diesem Medium, insbesondere auch keine Nutzungshäufigkeiten, erhoben. Damit wurde in dieser Studie für das Merkmal Zugang nicht (allein) die quantitative Ausstattung an Geräten gewählt.

Weiter wurde ein einfacher Indikator zu schulinternen Regelungen erhoben („An meiner Schule gibt es eine vs. gibt es keine einheitliche CAS/DGS-Regelung"). Solche Regelungen sollen in vielen Bundesländern von den Fachkonferenzen festgelegt werden (z. B. in Schleswig-Holstein) und etwa die Rechtsicherheit bei Prüfungen gewährleisten. Es kann davon ausgegangen werden, dass der Medieneinsatz an Schulen mit einheitlicher Regelung grundlegender und verbindlicher geklärt ist als an Schulen ohne erarbeitete Regelungen. Daher wurde das Vorhandensein schulinterner Regelungen bzgl. der genutzten Geräte für CAS und DGS als Indikator für fachspezifische schulinterne Rahmenbedingungen erhoben, der über den reinen Zugang hinaus informativ für das fachspezifische Medien-Schulklima ist.

Die Mediennutzungshäufigkeit in Bezug auf CAS und DGS wurde in Eigenentwicklung operationalisiert. Ähnlich wie bei Sailer, Mürbock und Fischer (2017) wurden Lernaktivitäten von Schülerinnen und Schülern mit DGS/CAS identifiziert, die die verschiedenen Funktionen schulischer Mediennutzung abbilden (Beitrag zur Grundbildung, Entwicklung von Medienkompetenz, instrumentelle Nutzung im Fachunterricht; Kerres, 2000). Die Lernaktivitäten im Bereich der instrumentellen Nutzung wurden dabei im Anschluss an den mathematikdidaktischen Diskurs bestimmt: Während das Erstellen eigener Berechnungen/Konstruktionen und deren Überprüfung grundlegende Funktionen nutzt, besteht das Potenzial von CAS und DGS darin, Visualisierungen zu erstellen sowie Gesetzmäßigkeiten zu entdecken (zsf. Schmidt-Thieme \& Weigand, 2010). Die Erhebung der Nutzung als Häufigkeit dieser Lernaktivitäten wurde je an ein kanonisches, für den Medieneinsatz besonders geeignetes Themenbeispiel (DGS: Strahlensätze, CAS: Lösen quadratischer Gleichungen) gekoppelt, um eine möglichst gute Vergleichbarkeit zwischen den Angaben der Lehrkräfte $\mathrm{zu}$ erzielen. Für jede der Lernaktivitäten wurden die Lehrkräfte aufgefordert, die auf die Unterrichtseinheit bezogenen Nutzungshäufigkeiten anzugeben (vgl. Tab. 2 auf der folgenden Seite). Dabei wurde eine Skalierung gewählt, die die typische Planungseinheit „Unterrichtsstunde“ nutzt ( $1=$ „in keiner oder fast keiner“", 2 = „in 
weniger als der Hälfte“, 3 = „in mindestens der Hälfte“, 4 = „in jeder oder fast jeder Unterrichtsstunde“). Weitere Lernaktivitäten konnten über offene Antwortformate ergänzt werden.

Tab. 2: Übersicht über die Konzeption der Lernaktivitäten zur Erhebung der Nutzungshäufigkeit

\begin{tabular}{ll}
\hline Fokus & Lernaktivität \\
& (Frage: „Wie häufig führen Ihre Schülerinnen und Schüler folgende \\
& $\begin{array}{l}\text { Lernaktivitäten typischerweise bei der Nutzung von DGS / CAS im } \\
\text { Mathematikunterricht zum Thema "'Strahlensätze” / „Lösen } \\
\text { quadratischer Gleichungen” durch?") }\end{array}$ \\
\hline Instrumentelle & zum Überprüfen eigener Berechnungen. \\
Nutzung & zur Konstruktion / Berechnung einer Lösung. \\
& zur Visualisierung eines Sachverhalts. \\
& zum Herausfinden von Zusammenhängen und Regeln. \\
Grundbildung & zum Lernen der Bedienung des DGS/CAS. \\
Medienkompetenz & zur Reflexion des mathematischen Hintergrunds der DGS-/CAS- \\
& Funktionalitäten. \\
\hline
\end{tabular}

Quelle: eigene Darstellung

\subsection{Methode}

Um die Frage nach der Nutzungshäufigkeit zu beantworten, wurden die Angaben zu CAS bzw. DGS aggregiert (vgl. Kap. 5.2). In einem zweiten Schritt wurde eine multinomiale logistische Regression (MLR) durchgeführt (abhängige Variable: Nutzungshäufigkeit), um die Effekte von Personenmerkmalen und organisationalen Merkmalen zu untersuchen (Variableneinschluss schrittweise vorwärts).

\section{Ergebnisse}

\subsection{Deskriptiva}

Für CAS gaben 41 Lehrkräfte an, über keinen Zugang zu verfügen, bei DGS waren es drei Lehrkräfte. Abbildung 2 auf der folgenden Seite zeigt die Häufigkeitsverteilungen der Angaben zum CAS- bzw. DGS-Zugang für die Lehrkräfte mit Zugang. Die Antworten wurden für die weitere Verwendung gruppiert. Dabei wurde unterschieden, ob für die Lehrkräfte der Einsatz von CAS bzw. DGS mit einem erhöhten unterrichts-organisatorischen Aufwand verbunden ist (Code 0, Buchung/Umzug in den Computerraum notwendig oder eingeschränkte Geräteverfügbarkeit) oder ob es keine erhöhte Zugangshürde gibt (Code 1). 
Abb. 2: Häufigkeitsverteilung der Angaben zum Zugang zu CAS $(N=144)$ bzw. DGS $(N=160)$

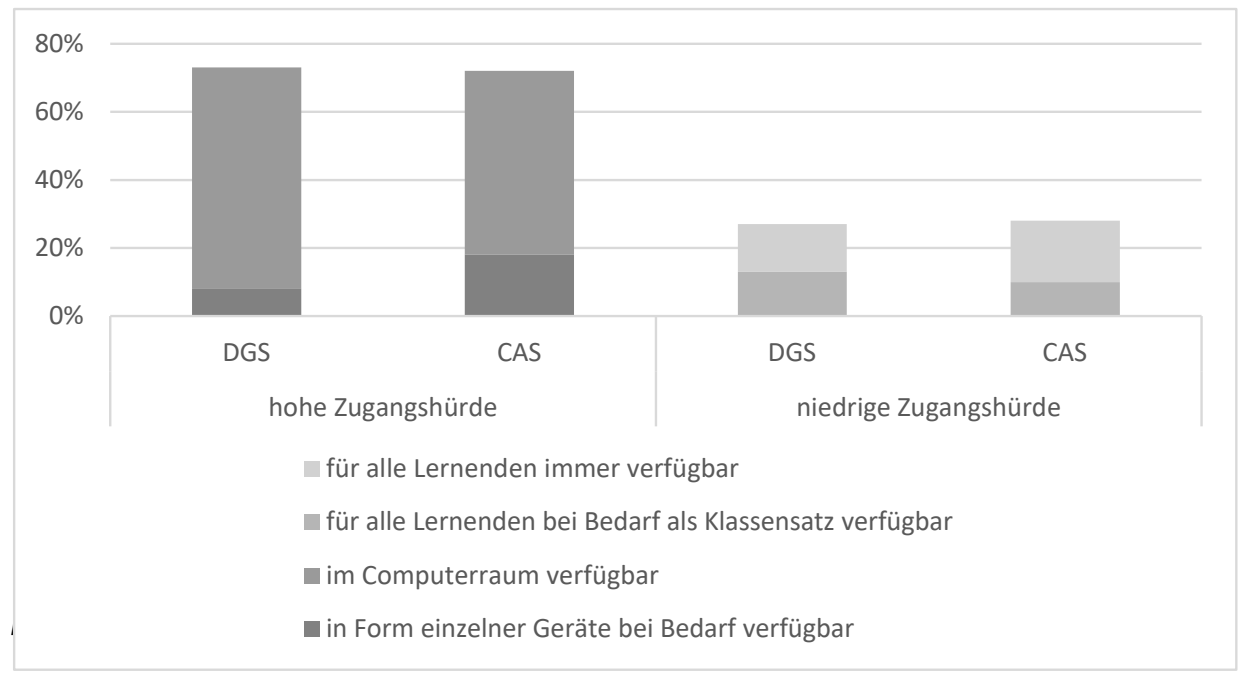

Quelle: eigene Darstellung

Dass an ihren Schulen keine schulinternen Regelungen bezüglich der Gerätewahl bestehen, berichten 40,4 Prozent (für CAS) bzw. 43,4 Prozent (für DGS) der Lehrkräfte.

Die Mittelwerte und Standardabweichungen der Skalen zu Einstellungen (5-stufiges Antwortformat) und Selbstwirksamkeitserwartung (4-stufig) gegenüber dem Einsatz digitaler Medien im Unterricht sind in Tabelle 3 aufgeführt. Die Angaben zu den Einstellungen sind hoch, sodass sich ein leichter Deckeneffekt zeigt.

Tab. 3: Mittelwerte und Standardabweichungen der Skalen Einstellungen und Selbstwirksamkeitserwartung

\begin{tabular}{lcccccc}
\hline Skala & $\begin{array}{c}\text { Item- } \\
\text { anzahl }\end{array}$ & $\begin{array}{c}\text { Cronbachs } \\
\text { Alpha }\end{array}$ & MW & SD & Minimum & Maximum \\
\hline Einstellungen & 6 & .83 & 3.96 & 0.66 & 2 & 5 \\
$\begin{array}{l}\text { Selbstwirksam- } \\
\text { keitserwartungen }\end{array}$ & 6 & .77 & 2.77 & 0.54 & 1 & 4 \\
\hline
\end{tabular}

Quelle: eigene Berechnung 


\subsection{Wie stellt sich die Nutzungshäufigkeit von CAS und DGS dar (Forschungsfrage 1)?}

In die Auswertung gingen nur Daten von Lehrkräften ein, die Zugang zu DGS bzw. CAS haben $\left(n_{C A S}=103, n_{D G S}=156\right)$. Die Kategorie 4 (,in jeder oder fast jeder Unterrichtsstunde“) war kaum besetzt, sodass sie mit der Kategorie 3 zusammengefasst wurde. Dies lässt sich inhaltlich begründen: Wenn ein System in Verbindung mit einer Lernaktivität in mindestens der Hälfte der Unterrichtsstunden benutzt wird, kann davon ausgegangen werden, dass die Schüler*innen es als gewohnten Bestandteil des Mathematikunterrichts erleben. Die entstehende Kategorie bezeichnen wir als Kategorie 3 (durchgängige Nutzung). Die Nutzungshäufigkeit ist damit in drei Abstufungen pro Lernaktivität erfasst: $1=($ fast $)$ keine Nutzung ${ }^{1}, 2$ = gelegentliche Nutzung und 3 =durchgängige Nutzung. Die Angaben variieren zwar zwischen den Lerngelegenheiten und lassen auch auf unterschiedliche Nutzungsintensitäten der Lehrkräfte schließen, aber es zeigen sich empirisch keine unterschiedlichen Nutzungsprofile. Daher wurden die Angaben für die folgenden Analysen so aggregiert, dass die Nutzungsintensität abgebildet ist. Dazu wurde pro Lehrkraft die höchste angegebene Nutzungshäufigkeit ermittelt und die Lehrkraft damit der Gruppe der Nicht-Nutzung, der gelegentlichen Nutzung oder der durchgängigen Nutzung zugeordnet. Die aggregierte Nutzungshäufigkeit spiegelt also die höchste Angabe jeder Lehrkraft. Die Verteilungen der Nutzungshäufigkeit von DGS/CAS sind Abbildung 3 zu entnehmen.

Abb. 3: Nutzungshäufigkeiten von CAS und DGS nach Lernaktivitäten

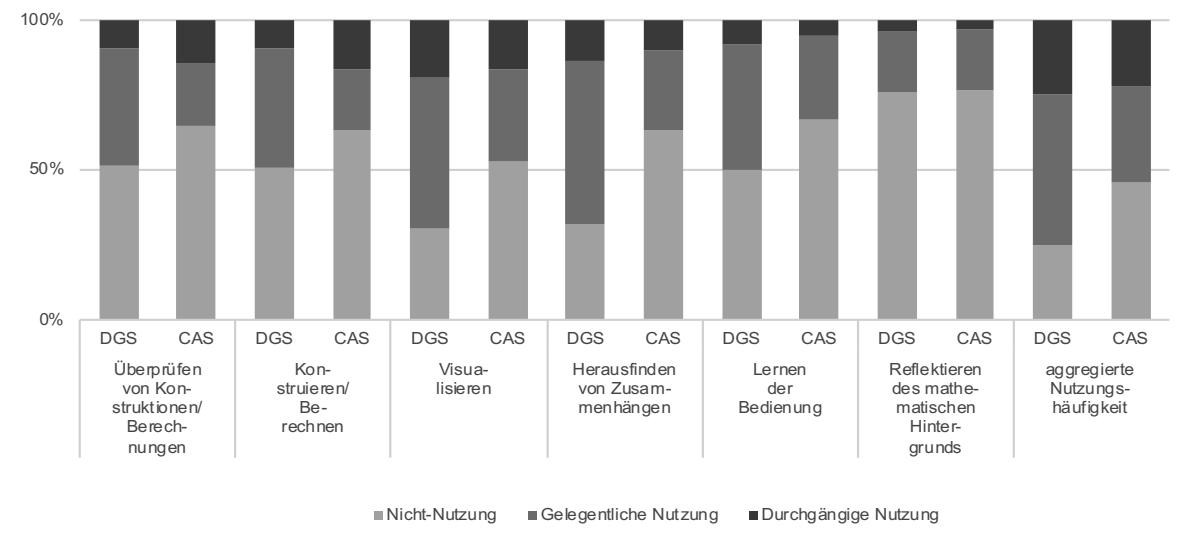

Quelle: eigene Darstellung

1 Im Folgenden wird zur besseren Lesbarkeit von der Nicht-Nutzung gesprochen. 
Nur wenige Lehrkräfte berichten von einer durchgängigen Nutzung, wobei sich der geringere Verpflichtungsgrad von CAS gegenüber DGS erwartungsgemäß in den aggregierten Nutzungshäufigkeiten niederschlägt.

\subsection{Welche Rolle spielen individuelle und organisationale Merkmale für die Nutzungshäufigkeit (Forschungsfrage 2)?}

Da DGS und CAS trotz unterschiedlichem Verbindlichkeitsgrad der gleichen Fachkultur unterliegen, ist zu vermuten, dass die Mechanismen, die zu Nutzungsunterschieden führen, dieselben sind. Deswegen wurde der Zusammenhang von Merkmalen der individuellen und der organisationalen Ebene mit der Nutzungshäufigkeit auf Basis der Daten zu beiden Systemen gemeinsam untersucht. Für den gestuften Vergleich der Gruppen "Nicht-Nutzung" mit "gelegentliche Nutzung" und "gelegentliche Nutzung" mit „durchgängige Nutzung" wurden zwei MLRs durchgeführt, die sich in den Referenzgruppen unterschieden (Referenz Nicht-Nutzung bzw. gelegentliche Nutzung). Es werden hier die Ergebnisse für benachbarte Gruppen berichtet.

In das finale Modell gingen die schulinternen Regelungen bezüglich DGS/CAS, der Zugang zu CAS/DGS sowie die Selbstwirksamkeitserwartung in Bezug auf das Unterrichten mit digitalen Medien als signifikante Prädiktoren ein $\left(\mathrm{R}_{\text {Cox\&snell }}^{2}=.20\right.$, $\mathrm{R}^{2}{ }_{\text {Nagelkerke }}=.22$, Modell $\left.\chi^{2}(6)=49.86, \mathrm{p}<.001\right)$. Nicht aufgenommen wurde die Einstellungs-Skala, da diese nicht zur Erklärung der Nutzungshäufigkeit beitrug.

\subsubsection{Gelegentliche vs. Nicht-Nutzung}

In dieser Studie erhöht das Vorhandensein schulinterner Regelungen bzgl. der genutzten Geräte die Wahrscheinlichkeit, dass Lehrkräfte Medien gelegentlich im Unterricht einsetzen (vgl. Tab. 4 auf der folgenden Seite). Das Odds Ratio von 4.06 deutet an, dass im Vergleich eine Lehrkraft, deren Schule interne Regelungen hat, mit einer vierfach höheren Chance zur Gruppe "gelegentliche Nutzung" statt zur Gruppe „Nicht-Nutzung“ zählt $(\mathrm{p}<.001)$. Die Selbstwirksamkeitserwartung und die Zugangsmerkmale zeigen in diesem Kontrast keinen Effekt. Das Vorhandensein schulinterner Regelungen scheint den Einstieg in die Nutzung der Systeme zu erleichtern.

\subsubsection{Durchgängige vs. Gelegentliche Nutzung}

Wird die durchgängige Nutzung mit der gelegentlichen Nutzung kontrastiert, steigt die Wahrscheinlichkeit, Medien durchgängig zu nutzen, um etwa das Vierfache, wenn 
die Zugangshürde zu den CAS/DGS niedrig ist (vgl. Tab. 4). Zudem zeigt sich ein Effekt der Selbstwirksamkeitserwartung in Bezug auf das Unterrichten mit digitalen Medien. Schulinterne Regelungen zeigen im Kontrast durchgängiger Nutzung mit gelegentlicher Nutzung keinen signifikanten Effekt. Förderlich für eine durchgängige Nutzung im Kontrast zur gelegentlichen Nutzung erscheinen ein einfacher Zugang zu den Systemen und die Einschätzung, mit digitalen Medien gut unterrichten zu können.

Tab. 4: Parameterschätzer der Gruppenvergleiche

\begin{tabular}{|c|c|c|c|c|c|c|}
\hline & \multicolumn{3}{|c|}{$\begin{array}{l}\text { Nicht-Nutzunga vs. } \\
\text { gelegentliche Nutzung }\end{array}$} & \multicolumn{3}{|c|}{$\begin{array}{l}\text { gelegentliche Nutzunga vs. } \\
\text { durchgängige Nutzung }\end{array}$} \\
\hline & $\stackrel{\mathrm{b}}{(S E)}$ & $\begin{array}{l}\text { Odds } \\
\text { Ratio }\end{array}$ & $p$-Wert & $\begin{array}{l}\mathrm{b} \\
(S E)\end{array}$ & $\begin{array}{l}\text { Odds } \\
\text { Ratio }\end{array}$ & $p$-Wert \\
\hline Konstanter Term & $\begin{array}{l}-.93 \\
(0.88)\end{array}$ & & .290 & $\begin{array}{l}-3.85 \\
(1.09)\end{array}$ & & $<.001$ \\
\hline $\begin{array}{l}\text { Vorhandensein } \\
\text { schulinterner Re- } \\
\text { gelungen }\end{array}$ & $\begin{array}{l}1.40 \\
(0.36)\end{array}$ & 4.06 & $<.001$ & $\begin{array}{l}-0.56 \\
(0.40)\end{array}$ & 0.57 & .153 \\
\hline Zugang & $\begin{array}{l}-0.26 \\
(0.41)\end{array}$ & 0.77 & .520 & $\begin{array}{l}1.38 \\
(0.38)\end{array}$ & 3.99 & $<.001$ \\
\hline $\begin{array}{l}\text { Selbstwirksam- } \\
\text { keitserwartung }\end{array}$ & $\begin{array}{l}0.26 \\
(0.32)\end{array}$ & 1.30 & .422 & $\begin{array}{l}1.07 \\
(0.36)\end{array}$ & 2.92 & .003 \\
\hline
\end{tabular}

Anm.: $\quad{ }^{a}$ Referenzkategorie

Quelle: eigene Berechnung

\section{Diskussion}

Wie stellt sich die Nutzung digitaler mathematikspezifischer Medien am Beispiel von CAS und DGS aktuell dar? Die Ergebnisse der Lehrkräftebefragung zeigen, dass vielfach noch nicht von einer durchgängigen Nutzung der Systeme auszugehen ist, wobei sich der unterschiedliche Verbindlichkeitsgrad von DGS und CAS in den Angaben der Lehrkräfte spiegelt. Zu beachten ist dabei, dass eine hohe Nutzungshäufigkeit noch keinen Schluss auf die unterrichtliche Qualität des Medieneinsatzes zulässt, da für die Beurteilung der Qualität der lernzielorientierte Einsatz zu berücksichtigen ist (Petko, 2012). Mit einer sporadischen Nutzung der durchaus komplexen Systeme können die in den Curricula intendierten Lernziele aber sicher nicht erreicht werden.

Die Ergebnisse zu den Bedingungsfaktoren deuten an, dass, ähnlich den Erkenntnissen aus fachunspezifischer Sicht, auch für die Nutzung mathematikspezifischer Medien organisationale Merkmale und Personenmerkmale zur Erklärung von Nutzungsunterschieden beitragen. Die Studie kann darüber hinaus Einsichten zur 
Relevanz verschiedener Faktoren im gestuften Vergleich Nicht-Nutzung - gelegentliche Nutzung - durchgängige Nutzung geben.

Auf individueller Ebene gehen in dieser Studie Einstellungen gegenüber den Medien als Prädiktor für die Mediennutzung nicht in das finale Modell ein, obwohl sie aus handlungstheoretischer Sicht relevant sind (zsf. Davis, 1989). Da für die Einstellungen in der vorliegenden Studie teils Deckeneffekte auftraten, differenzieren diese möglicherweise nicht ausreichend, um über die anderen Variablen hinaus Unterschiede in den Nutzungshäufigkeiten erklären zu können. Zu berücksichtigen ist dabei, dass die Einstellungen nicht medienspezifisch erhoben wurden. Es muss daher an dieser Stelle offenbleiben, ob Einstellungen gegenüber mathematikspezifischen Medien sich gegebenenfalls als zusätzliche Prädiktoren eignen.

Wie zu erwarten, trägt in dieser Studie die Selbstwirksamkeitserwartung der Lehrkräfte bezüglich des Medieneinsatzes zur Erklärung der Nutzungshäufigkeit bei. Jedoch wird die Selbstwirksamkeitserwartung erst im Vergleich der gelegentlichen mit der durchgängigen Nutzung relevant. Dasselbe gilt auch für Merkmale des Zugangs zu den mathematikspezifischen Medien, sodass niedrige organisationale Hürden erst für Unterschiede zwischen gelegentlicher und durchgängiger Nutzung relevant scheinen, nicht jedoch für die zwischen Nicht-Nutzung und gelegentlicher Nutzung. Einschränkend ist festzuhalten, dass weitere, in umfassenderen Studien als relevant identifizierte Merkmale auf organisationaler Ebene in dieser Studie nicht erhoben wurden (z. B. Qualität der Ausstattung, technischer Support).

Für die Erklärung von Unterschieden zwischen der Nicht-Nutzung und der gelegentlichen Nutzung erweisen sich in unserer Studie deutlich schulinterne fachspezifische Regelungen als wichtig. Es kann davon ausgegangen werden, dass das Vorhandensein solcher Regelungen impliziert, dass sich das Fachkollegium über die Geräte ausgetauscht hat, Vereinbarungen zur Nutzung in Klassenarbeiten ausgehandelt und die Geräte und deren Funktionen bestenfalls in das schulinterne Fachcurriculum eingegliedert sind. Deswegen verstehen wir die Frage nach den Regelungen bezüglich genutzter Geräte als einen Indikator für die an der Schule herrschende Kultur in Bezug auf CAS- bzw. DGS-Nutzung. Dies könnte seine Wirkung als Prädiktor im Einklang mit den Befunden zur Relevanz von schulorganisatorischen Merkmalen wie gemeinsamen Zielen und Visionen erklären.

Zu den Limitationen der Studie gehört, dass die Stichprobe eine Gelegenheitsstichprobe und somit nicht repräsentativ ist, auch wenn nicht von einer systematischen Verzerrung der Stichprobe im Vergleich zu einer repräsentativen Lehrkräftebefragung auszugehen ist. Weiter basiert die Studie auf Selbstberichten, sodass insbesondere keine objektivierbaren Angaben zu Nutzungshäufigkeiten vorliegen. Die Erhebung wurde zwar mit Bezug zu kanonischen Unterrichtsthemen durchgeführt, um die Validität relativer Vergleiche zu erhöhen, jedoch wissen wir nicht, wann die Lehrkräfte diese 
Themen zuletzt unterrichtet haben. Inwiefern die Generalisierbarkeit der Ergebnisse durch die nicht repräsentative Stichprobe und eingeschränkte Themenwahl beeinträchtigt ist, ist jedoch kaum abzuschätzen, da allgemein Daten zur Nutzung fachspezifischer Medien fehlen. Die Vermutung liegt aber nahe, dass die Nutzungsangaben die aktuelle Lage eher überschätzen, da angenommen werden kann, dass die Stichprobe insgesamt aus eher engagierten Lehrkräften besteht.

Für die Praxis lassen sich mehrere Ansatzpunkte zur Förderung von Mediennutzung im Mathematikunterricht begründen. Einerseits deuten die Ergebnisse an, dass Strategien zur Lehrkräftequalifizierung, die zu einer erhöhten Selbstwirksamkeitserwartung beitragen, sowie eine Senkung der Zugangshürden zu den Geräten förderlich sein können, um die Nutzungshäufigkeit von Medien im Fachunterricht zu steigern, wenn diese bereits genutzt werden. Andererseits legen die Ergebnisse nahe, dass Strategien, die auf Ebene der Schule und der Fachkollegien mit dem Ziel der Veränderung einer medienspezifischen schulinternen Fachkultur ansetzen, bei der Integration von Medien im Mathematikunterricht helfen können. In der Studie bildet sich das im relativ erklärmächtigen Indikator der schulinternen Regelungen in Bezug auf die genutzten Geräte für CAS und DGS ab. Inwiefern unspezifischere Maßnahmen, wie z.B. fachübergreifende Medienkonzepte, ebenfalls zur Erklärung der mathematikspezifischen Mediennutzung beitragen können, kann diese Studie nicht beantworten. Die Ergebnisse deuten aber an, dass eine Veränderung der fachspezifischen Medienkultur gerade für den Einstieg in die Nutzung mathematikspezifischer Medien hilfreich ist. Insgesamt implizieren die Ergebnisse der Studie, dass fachspezifische Maßnahmen, die auf der organisationalen Ebene ansetzen, mit solchen, die die individuelle Ebene adressieren, Hand in Hand gehen sollten.

Wir danken der Joachim Herz Stiftung für die Förderung des Projekts „MiU - Medieneinsatz im mathematisch-naturwissenschaftlichen Unterricht", in dessen Rahmen die Befragung stattfand, sowie allen Teilnehmenden der Befragung.

\section{Literatur und Internetquellen}

Ackeren, I. van, Aufenanger, S., Eickelmann, B., Friedrich, S., Kammerl, R., Knopf, J., et al. (2019). Digitalisierung in der Lehrerbildung. Herausforderungen, Entwicklungsfelder und Förderung von Gesamtkonzepten. DDS - Die Deutsche Schule, 111 (1), 103-119. https://doi.org/10.31244/dds.2019.01.10

Barzel, B. (2012). Computeralgebra im Mathematikunterricht: Ein Mehrwert - aber wann? Münster: Waxmann.

BITKOM (2015). Digitale Schule - vernetztes Lernen. Ergebnisse repräsentativer Schülerund Lehrerbefragungen zum Einsatz digitaler Medien im Schulunterricht. Zugriff am 22.04.2020. Verfügbar unter: https://www.bitkom.org/Bitkom/Publikationen/DigitaleSchule-und-vernetztes-Lernen.html.

Davis, F. D. (1989). Perceived Usefulness, Perceived Ease of Use, and User Acceptance of Information Technology. MIS Quarterly, 13, 319-340. https://doi.org/10.2307/249008 
Drossel, K., Eickelmann, B., \& Gerick, J. (2017). Predictors of Teachers' Use of ICT in School - the Relevance of School Characteristics, Teachers' Attitudes and Teacher Collaboration. Education and Information Technologies, 22 (2), 551-573. https://doi. org/10.1007/s10639-016-9476-y

Eickelmann, B., Bos, W., Gerick, J., Goldhammer, F., Schaumburg, H., Schwippert, K. et al. (2019). ICILS 2018 \#Deutschland. Computer- und informationsbezogene Kompetenzen von Schülerinnen und Schülern im zweiten internationalen Vergleich und Kompetenzen im Bereich Computational Thinking. Münster: Waxmann.

Eickelmann, B., Gerick, J., \& Koop, C. (2017). ICT Use in Mathematics Lessons and the Mathematics Achievement of Secondary School Students by International Comparison: Which Role do School Level Factors Play? Education and Information Technologies, 22 (4), 1527-1551. https://doi.org/10.1007/s10639-016-9498-5

Fahlgren, M., \& Brunström, M. (2014). A Model for Task Design with Focus on Exploration, Explanation, and Generalization in a Dynamic Geometry Environment. Technology, Knowledge and Learning, 19 (3), 287-315. https://doi.org/10.1007/s10758-014-9213-9

Handal, B., Cavanagh, M., Wood, L., \& Petocz, P. (2011). Factors Leading to the Adoption of a Learning Technology: The Case of Graphics Calculators. Australasian Journal of Educational Technology, 27 (2), 343-360. https://doi.org/10.14742/ajet.974

Härtig, H., Kampschulte, L., Lindmeier, A., Ostermann, A., Ropohl, M., \& Schwanewedel, J. (2018). Wie lässt sich Medieneinsatz im Fachunterricht beschreiben? In M. Ropohl, A. Lindmeier, H. Härtig, L. Kampschulte, A. Mühling \& J. Schwanewedel (Hrsg.), Medieneinsatz im mathematisch-naturwissenschaftlichen Unterricht (S. 157-192). Hamburg: Joachim Herz Stiftung.

Hermans, R., Tondeur, J., van Braak, J., \& Valcke, M. (2008). The Impact of Primary School Teachers' Educational Beliefs on the Classroom Use of Computers. Computers \& Education, 51 (4), 1499-1509. https://doi.org/10.1016/j.compedu.2008.02.001

Hillmayr, D., Reinhold, F., Ziernwald, L., \& Reiss, K. (2017). Digitale Medien im mathematisch-naturwissenschaftlichen Unterricht der Sekundarstufe: Einsatzmöglichkeiten, Umsetzung und Wirksamkeit. Münster: Waxmann.

Hoffkamp, A. (2011). The Use of Interactive Visualizations to Foster the Understanding of Concepts of Calculus: Design Principles and Empirical Results. ZDM, 43 (3), 359-372. https://doi.org/10.1007/s11858-011-0322-9

Huberman, M. (1991). Der berufliche Lebenszyklus von Lehrern. Ergebnisse einer empirischen Untersuchung. In E. Terhart (Hrsg.), Unterrichten als Beruf. Neuere amerikanische und englische Arbeiten zur Berufskultur und Berufsbiographie von Lehrerinnen und Lehrern (S. 249-267). Köln: Böhlau.

KMK (Sekretariat der Ständigen Konferenz der Kultusminister der Länder in der Bundesrepublik Deutschland). (2015). Bildungsstandards im Fach Mathematik für die Allgemeine Hochschulreife. Beschluss der KMK vom 18.10.2012. Berlin.

KMK (Sekretariat der Ständigen Konferenz der Kultusminister der Länder in der Bundesrepublik Deutschland). (2016). Strategie der Kultusministerkonferenz „Bildung in der digitalen Welt“. Beschluss der Kultusministerkonferenz vom 8.12.2016. Berlin.

Lindau, B., Kübler, S., \& Spada H. (2013). Entwicklung und Überprüfung eines Modells der Bereitschaft zum Medien- und Technologieeinsatz bei weiblichen und männlichen Lehramtsstudierenden. Unterrichtswissenschaft, 41, 20-37.

Lorenz, R., Bos, W., \& Endberg, M. (2017). Schule digital - der Länderindikator 2017. Schulische Medienbildung in der Sekundarstufe I mit besonderem Fokus auf MINTFächer im Bundesländervergleich und Trends von 2015 bis 2017. Münster: Waxmann.

Meinhardt, C., Rabe, T., \& Krey, O. (2016). Selbstwirksamkeitserwartungen in physikdidaktischen Handlungsfeldern. Skalendokumentation. Version 1.0 (Februar 2016). Zugriff am 10.04.2020. Verfügbar unter: http://nbn-resolving.de/urn:nbn:de:0111-pedocs-118180. 
Petko, D. (2012). Teachers' Pedagogical Beliefs and their Use of Digital Media in Classrooms: Sharpening the Focus of the 'Will, Skill, Tool' Model and Integrating Teachers' Constructivist Orientations. Computers \& Education, 58, 1352-1359. https://doi. org/10.1016/j.compedu.2011.12.013

Prasse, D. (2012). Bedingungen innovativen Handelns in Schulen. Funktion und Interaktion von Innovationsbereitschaft, Innovationsklima und Akteursnetzwerken am Beispiel der IKT-Integration an Schulen. Münster: Waxmann.

Rieß, M. (2018). Zum Einfluss digitaler Werkzeuge auf die Konstruktion mathematischen Wissens. Wiesbaden: Springer. https://doi.org/10.1007/978-3-658-20644-4

Rolfes, T., Roth, J., \& Schnotz, W. (2020). Learning the Concept of Function with Dynamic Visualizations. Frontiers in Psychology, 11, 693. https://doi.org/10.3389/fpsyg.2020. 00693

Sailer, M., Murböck, J., \& Fischer, F. (2017). Digitale Bildung an bayerischen Schulen Infrastruktur, Konzepte, Lehrerbildung und Unterricht. München: vbw.

Schmidt-Thieme, B., \& Weigand, H.-G. (2015). Medien. In R. Bruder, L. Hefendehl-Hebeker, B. Schmidt-Thieme \& H.-G. Weigand (Hrsg.), Handbuch der Mathematikdidaktik (S. 461-490). Berlin: Springer. https://doi.org/10.1007/978-3-642-35119-8_17

Schmitz, G. S., \& Schwarzer, R. (2000). Selbstwirksamkeitserwartung von Lehrern: Längsschnittbefunde mit einem neuen Instrument. Zeitschrift für Pädagogische Psychologie, 14 (1), 12-25. https://doi.org/10.1024//1010-0652.14.1.12

Thomas, M. O. J., \& Palmer, J. M. (2014). Teaching with Digital Technology: Obstacles and Opportunities. In A. Clark-Wilson, O. Robutti \& N. Sinclair (Hrsg.), The Mathematics Teacher in the Digital Era: An International Perspective on Technology Focused Professional Development (S. 71-89). Dordrecht: Springer Netherlands. https://doi. org/10.1007/978-94-007-4638-1_4

Thurm, D. (2020). Digitale Werkzeuge im Mathematikunterricht integrieren. Zur Rolle von Lehrerüberzeugungen und der Wirksamkeit von Fortbildungen. Wiesbaden: Springer. https://doi.org/10.1007/978-3-658-28695-8

Tondeur, J., van Keer, H., van Braak, J., \& Valcke, M. (2008). ICT Integration in the Classroom: Challenging the Potential of a School Policy. Computers \& Education, 51 (1), 212-223. https://doi.org/10.1016/j.compedu.2007.05.003

Venturini, M., \& Sinclair, N. (2017). Designing Assessment Tasks in a Dynamic Geometry Environment. In A. Leung \& A. Baccaglini-Frank (Hrsg.), Digital Technologies in Designing Mathematics Education Tasks (S.77-98). Cham: Springer International Publishing. https://doi.org/10.1007/978-3-319-43423-0_5

Weigand, H.-G., \& Bichler, E. (2010). Towards a Competence Model for the Use of Symbolic Calculators in Mathematics Lessons: The Case of Functions. ZDM, 42 (7), 697-713. https://doi.org/10.1007/s11858-010-0274-5

Anje Ostermann, geb. 1991, Doktorandin am Leibniz-Institut für die Pädagogik der Naturwissenschaften und Mathematik in Kiel.

E-Mail: ostermann@leibniz-ipn.de

Korrespondenzadresse: Olshausenstraße 62, 24118 Kiel

Hendrik Härtig, Prof. Dr., geb. 1979, Professor für Didaktik der Physik an der Universität Duisburg-Essen.

E-Mail: hendrik.haertig@uni-due.de

Korrespondenzadresse: Universitätsstr. 2, 45141 Essen 
Lorenz Kampschulte, Dr., geb. 1976, Leiter der Hauptabteilung Bildung am Deutschen Museum in München.

E-Mail: 1.kampschulte@deutsches-museum.de

Korrespondenzadresse: Museumsinsel 1, 80538 München

Mathias Ropohl, Prof. Dr., geb. 1981, Professor für Didaktik der Chemie an der Universität Duisburg-Essen.

E-Mail: mathias.ropohl@uni-due.de

Korrespondenzadresse: Schützenbahn 70, 45127 Essen

Julia Schwanewedel, Prof. Dr., geb. 1979, Professorin für Didaktik der Naturwissenschaften - Biologie an der Universität Hamburg.

E-Mail: julia.schwanewedel@uni-hamburg.de

Korrespondenzadresse: Von-Melle-Park 8, 20146 Hamburg

Anke Lindmeier, Prof. Dr., geb. 1979, Professorin für Didaktik der Mathematik an der Friedrich-Schiller-Universität Jena.

E-Mail: anke.lindmeier@uni-jena.de

Korrespondenzadresse: Ernst-Abbe-Platz 2, 07743 Jena 


\section{Anhang}

\section{Skala „Selbstwirksamkeitserwartung beim Unterrichten mit digitalen Werkzeugen“}

\section{Verwendete Skala}

\begin{tabular}{|c|c|c|c|c|c|}
\hline & $\begin{array}{l}\text { Bitte bewerten Sie, inwiefern Sie den folgenden Aussagen } \\
\text { zustimmen. }\end{array}$ & 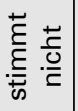 & 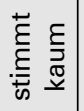 & 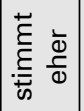 & 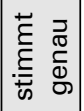 \\
\hline digM1 & $\begin{array}{l}\text { Ich kann in meiner Unterrichtsplanung zu den Lernzielen } \\
\text { passende Einsätze digitaler Medien planen, auch wenn mei- } \\
\text { ne Schule nicht optimal mit digitalen Medien ausgestattet } \\
\text { ist. }\end{array}$ & $\square$ & $\square$ & $\square$ & $\square$ \\
\hline digM2 & $\begin{array}{l}\text { Ich kann den Einsatz digitaler Medien im Fachunterricht so } \\
\text { planen, dass meine Schülerinnen und Schüler begeistert } \\
\text { sind, auch wenn sie sich sonst wenig für das Fach interes- } \\
\text { sieren. }\end{array}$ & $\square$ & $\square$ & $\square$ & $\square$ \\
\hline digM3 & $\begin{array}{l}\text { Ich kann den Einsatz eines digitalen Mediums im Fachunter- } \\
\text { richt didaktisch begründen, auch wenn ich dieses digitale } \\
\text { Medium noch nicht selbst eingesetzt habe. }\end{array}$ & $\square$ & $\square$ & $\square$ & $\square$ \\
\hline digM4 & $\begin{array}{l}\text { Ich kann eine Lösung für technische Probleme beim Unter- } \\
\text { richten mit digitalen Medien finden, auch wenn ich unter } \\
\text { Zeitdruck stehe. }\end{array}$ & $\square$ & $\square$ & $\square$ & $\square$ \\
\hline digM5 & $\begin{array}{l}\text { Ich kann den Einsatz digitaler Medien so gestalten, dass } \\
\text { meine Schülerinnen und Schüler motiviert sind mitzuar- } \\
\text { beiten, auch wenn es sich um eine unbeliebte Randstunde } \\
\text { handelt. }\end{array}$ & $\square$ & $\square$ & $\square$ & $\square$ \\
\hline digM6 & $\begin{array}{l}\text { Ich kann den fachlichen Lernprozess durch den Einsatz di- } \\
\text { gitaler Medien unterstützen, auch wenn unvorhergesehene } \\
\text { Verständnisschwierigkeiten auftreten. }\end{array}$ & $\square$ & $\square$ & $\square$ & $\square$ \\
\hline
\end{tabular}




\section{Konzeption der Skala}

\begin{tabular}{|l|l|l|}
\hline & \multicolumn{2}{|l|}{$\begin{array}{l}\text { Entsprechung nach Meinhardt, Rabe \& Krey (2016). Selbstwirksamkeitserwartungen } \\
\text { in physikdidaktischen Handlungsfeldern, Handlungsfeld Experimentieren (SWE-EX), } \\
\text { Dimensionen Planung (exp) und Durchführung (exd) }\end{array}$} \\
\hline digM1 & exp1 & $\begin{array}{l}\text { Ich kann in meiner Unterrichtsplanung zu den Lernzielen passende } \\
\text { Experimente aufbauen, auch wenn die Physiksammlung schlecht aus- } \\
\text { gestattet ist. }\end{array}$ \\
\hline digM2 & exp6 & $\begin{array}{l}\text { Ich kann ein Experiment planen, das meine Schülerinnen und Schüler } \\
\text { begeistert, auch wenn sie sich sonst wenig für Physik interessieren. }\end{array}$ \\
\hline digM3 & $\begin{array}{l}\text { Keine direkte Entsprechung, enthält den Aspekt der didaktischen Begründung ohne } \\
\text { hohe Vertrautheit wie in exp7 }\end{array}$ \\
\hline digM4 & exd5 & $\begin{array}{l}\text { Ich kann ein Experiment, das im Physikunterricht nicht auf Anhieb funk- } \\
\text { tioniert, zum Laufen bringen, auch wenn ich unter Zeitdruck stehe. }\end{array}$ \\
\hline digM5 & exd8 & $\begin{array}{l}\text { Ich kann ein Experiment so inszenieren, dass meine Schülerinnen und } \\
\text { Schüler motiviert sind mitzuarbeiten, auch wenn es sich um eine unbe- } \\
\text { liebte Randstunde handelt. }\end{array}$ \\
\hline digM6 & Keine direkte Entsprechung, enthält Aspekte von exd6, exd7 und exd9 \\
\hline
\end{tabular}




\section{DISKUSSION}

DDS - Die Deutsche Schule

113. Jahrgang 2021, Heft 2, S. 218-226

https://doi.org/10.31244/dds.2021.02.08

CC BY-NC-ND 4.0 Waxmann 2021

Hans-Jürgen Kuhn \& Michael Voges

\section{Blindflug beenden und stark aus der Krise kommen} Bildungschancen für Benachteiligte jetzt sichern! ${ }^{1}$

\section{Zusammenfassung}

Vor einem Jahr wurden Kitas und Schulen in Deutschland pandemiebedingt geschlossen. Nur in einigen Wochen im Sommer fand regelhafter Präsenzunterricht statt. Der stattdessen angebotene Fern-, Hybrid- und Wechselunterricht hat erhebliche Auswirkungen für Schüler*innen, Lehrkräfte, Eltern und Familien mit sich gebracht und hat zu Lernrückständen geführt; soziale Kontakte wurden reduziert und psychische und gesundheitliche Belastungen der an Schule direkt oder indirekt Beteiligten haben zugenommen. Die schon vor den coronabedingten Schulschließungen bestehenden Bildungsungerechtigkeiten haben sich pandemiebedingt weiter vergrößert. Damit sie nicht noch größer werden und sich auf lange Sicht weiter verfestigen, ist zügiges Handeln erforderlich. Im vorliegenden Beitrag werden zum einen gesicherte Erkenntnisse über die kognitiven, sozialen und psychischen Auswirkungen der pandemiebedingten Schulschließungen und zum anderen diagnosebasierte und wissenschaftich fundierte Förderkonzepte sowie ein umfassendes Förderprogramm gefordert und zur Diskussion gestellt, um gestärkt die Pandemie überwinden zu können.

Schlüsselwörter: Corona-Pandemie, Lernlücken, Kompetenzstände, Bund-Länder-Programm

1 Der Text beruht auf Diskussionen der Fokusgruppe Bildungspolitik der Heinrich-Böll-Stiftung und ist online verfügbar unter https://www.boell.de/de/themen/bildung-wissenschaft. Er richtet sich in erster Linie an die bildungspolitisch Verantwortlichen in Bund und Ländern und fokussiert auf Monitoring, Diagnostik und kompensatorische Förderung, für die es jetzt dringend politischer Entscheidungen bedarf. Uns ist bewusst, dass damit die Folgen der Pandemie für Schüler*innen, Lehrkräfte und Familien nur teilweise erfasst werden; das gilt auch für die Lösungen, die - auf der Grundlage einer verbesserten Datenlage - zum großen Teil noch entwickelt werden müssen. Die Redaktion der DDS dankt der HeinrichBöll-Stiftung für die Genehmigung des Nachdrucks, der hier mit nur minimalen redaktionellen Korrekturen erfolgt. 


\title{
Quitting the Blind Flight and Coming Out of the Crisis Strong
}

Secure Educational Opportunities for the Disadvantaged now!

\begin{abstract}
Last year, early childhood education and care facilities and schools in Germany were closed due to the Pandemic. Regular face-to-face teaching only happened during a few weeks in the summer. In its place, distance teaching, hybrid teaching, or alternate teaching were offered, entailing substantial consequences for students, teachers, parents, and families, and also causing learning gaps. Social contacts were reduced and there were massive strains on the psychological and physiological health of all those directly or indirectly involved in schools. Educational injustices existing before the Pandemic have increased. To keep them from growing further and becoming more stable, a rapid intervention is necessary. This paper demands and discusses reliable data on the cognitive, social, and psychological effects of the school closures, diagnose-based and scientifically sound support concepts, and a comprehensive support program for the educational system in order to overcome the Pandemic and emerge stronger.
\end{abstract}

Keywords: Corona Pandemic, learning gaps, competency levels, federal-state program

\section{Vorwort}

Vor einem Jahr, am 16. März 2020, wurden pandemiebedingt Kitas und Schulen in Deutschland geschlossen. Nach einigen Wochen mit teilweisem Präsenzunterricht im Sommer waren die meisten Schüler*innen auch im Schuljahr 2020/21 wieder überwiegend zu Hause ohne Präsenzunterricht.

Ein Jahr mit Öffnungen und Schließungen, Präsenz- und Fernunterricht, Hybridund Wechselunterricht liegt hinter uns, ein Jahr, das erhebliche Auswirkungen hatte für Schüler*innen, Lehrkräfte, Eltern und Familien: Lernrückstände, fehlende soziale Kontakte, psychische und gesundheitliche Belastungen. Gerade die ohnehin schon sozial und ökonomisch Benachteiligten haben besonders viel verloren. Schon vor Corona hatte unser Bildungssystem ein Gerechtigkeitsproblem, trotz Präsenzunterricht für alle. Seit mehr als einem Jahr können wir allerdings beobachten, wie sich die Ungleichheiten verschärfen, weil durch den Wegfall der täglichen Unterrichtssituation das Lern- und Sozialverhalten vieler Kinder und Jugendlichen zusätzlich beeinträchtigt wurde - mit fatalen Folgen. Damit die Chancenungleichheit nicht noch größer wird und sich auf lange Sicht verfestigt, muss zügig gehandelt werden.

Die jetzt erfolgte schrittweise Öffnung der Schulen wird spätestens zum Schuljahr 2021/22 eine Rückkehr in den regelhaften Präsenzunterricht möglich machen. Wer dann helfen und unterstützen will, muss zunächst wissen, wo das Problem liegt und wer von ihm betroffen ist. Daher muss der Blindflug schnellstmöglich been- 
det werden: Wir brauchen Klarheit über die Auswirkungen der pandemiebedingten Schulschließungen.

\section{Wir brauchen dringend gesicherte Erkenntnisse über die kognitiven, sozialen und psychischen Auswirkungen der pandemiebedingten Schulschließungen!}

Es gibt bis heute in Deutschland keine gesicherten Kenntnisse über die Auswirkungen der Pandemie auf die Schüler*innen der verschiedenen Altersgruppen: Welche kognitiven Lernlücken und sozialen Defizite, welche Brüche sind entstanden, was bedeuten zerbrochene Alltagsstrukturen, Brüche in Beziehungen zwischen Schüler ${ }^{\star}$ innen und Lehrkräften, Schüler*innen untereinander, Abbrüche in Lernverläufen, extremer Medienkonsum, Bewegungsmangel, problematische Ernährung, Verlust von Selbstvertrauen und zunehmende Isolation? Schule ist immer Lern- und Lebensort zugleich, mit vielfältigen Sozialisationseffekten, die zu Hause so nicht stattfinden und auch nicht einfach zu kompensieren sind.

Beobachtungen von Mediziner*innen, Psycholog*innen, Lehrkräften, Eltern und anderen Personen weisen auf zahlreiche Probleme hin, ersetzen aber keine systematischen Untersuchungen der verschiedenen Alterskohorten. Zwar hat sich seit dem Frühjahr 2020 um „Schule und Corona“ ein dynamisches Forschungsfeld herausgebildet (vgl. Fickermann \& Edelstein, 2020, 2021), dabei dominieren jedoch viele kleine Projekte, die vor allem als Ad-Hoc-Stichproben bei Online-Erhebungen durchgeführt wurden, sich eher auf technisch-organisatorische Fragen beim Fernunterricht fokussieren und meistens vor allem Lehrkräfte und Eltern befragen.

Bis heute gibt es keine repräsentative Studie für Deutschland, die die Auswirkungen der Schulschließungen auf die kognitive Kompetenzentwicklung beim Einsatz von Fern- und/oder Hybridunterricht nach Alterskohorten differenziert und belastbar beschreibt und längsschnittlich angelegt ist, um Veränderungen im Zeitverlauf messen zu können. Stattdessen werden zahlreiche, zum Teil auch weitreichende Lösungen zur Behebung einer Krise vorgeschlagen, für deren Wirksamkeit keine belastbaren empirischen Befunde vorliegen: Die (freiwillige oder angeordnete) Wiederholung des Schuljahres, verpflichtender Samstagsunterricht, Kürzung der Sommerferien, Verlängerung des Schuljahres bis Weihnachten 2021 machen entsprechend Furore.

Was die tatsächlichen Auswirkungen der Pandemie auf Schulen, Schüler*innen, Lehrkräfte und Familien angeht, befinden wir uns noch immer im Blindflug: Es bedarf dringend eines umfangreichen und koordinierten Forschungsprogramms, das systematisch und längsschnittlich die verschiedenen Aspekte der kognitiven und sozialen Kompetenzentwicklung unter Berücksichtigung von Herkunftsmerkmalen der 
Schüler*innen sowie der sozioökonomischen Lage der Familien in den Blick nimmt und versucht, Gelingensbedingungen zum Abbau von Bildungsungleichheit zu identifizieren.

Die Konferenz der Kultusminister*innen (KMK) und das Bundesministerium für Bildung und Forschung (BMBF) haben es bis heute versäumt, ein solches Forschungsprogramm für Deutschland zeitnah aufzulegen und ausreichend zu finanzieren. Deutschland verfügt u.a. mit dem Institut zur Qualitätsentwicklung im Bildungswesen (IQB), dem Leibniz-Institut für Bildungsforschung und Bildungsinformation (DIPF), dem Leibniz-Institut für die Pädagogik der Naturwissenschaften und Mathematik (IPN), dem Zentrum für internationale Bildungsvergleichsstudien (ZIB) und dem Leibniz-Institut für Bildungsverläufe (NEPS-Netzwerk) über exzellente wissenschaftliche Einrichtungen mit einer Expertise in Large Scale Assessments. Diese Kompetenzen müssen mit Blick auf ein konzertiertes, strategisches Handeln gebündelt und genutzt werden, wenn Bund und Länder künftige Steuerungs- und Gestaltungsmöglichkeiten nicht verspielen wollen!

Der IQB-Bildungstrend 2021, der im Mai 2021 die Lernstände aller Viertklässler*innen in den Bereichen Deutsch und Mathematik auf der Basis einer repräsentativen Studie erfassen soll, bietet die erste Möglichkeit, die Kompetenzstände der vermutlich besonders stark betroffenen Grundschüler*innen bundesweit zu untersuchen. Die Studie könnte um geeignete Aufgaben und Fragebögen ergänzt werden, um eine Einschätzung der pandemiebedingten Auswirkungen (Lernstände, psychische und soziale Folgen) zu erlauben. Wenn eine derartige Ergänzung des Bildungstrends 2021 jetzt aus zeitlichen Gründen nicht mehr realisiert werden kann, sollten die Länder zumindest eine ggf. im Umfang reduzierte Untersuchung zu Beginn der 5. Jahrgangsstufe beauftragen. Dabei sollten durch gezieltes Oversampling bestimmte Schülergruppen (z. B. Schüler*innen mit sonderpädagogischem Förderbedarf, Schüler*innen nicht deutscher Herkunftssprache) genauer untersucht werden, um dann in den folgenden Schuljahren gezielte Maßnahmen entwickeln zu können. Kinder und Jugendliche mit sonderpädagogischem Förderbedarf müssen dabei besonders berücksichtigt werden, ihre aktuelle Situation ist in der öffentlichen Diskussion weitgehend ausgeblendet worden! Diese Zusatzuntersuchungen sollten möglichst umgehend ausgewertet werden, um eine gezielte Förderstrategie für die jeweilige Personengruppe zu entwickeln.

Außer einer sehr begrüßenswerten Zusatzstudie im Nationalen Bildungspanel (NEPS), die im Frühjahr 2021 die Lernstände und Lernzuwächse von Neuntklässler*innen in Mathematik erfasst, gibt es derzeit für die Jahrgangsstufen der Sekundarstufe I und die Jugendlichen im Übergang in die Berufsausbildung keine Pläne, ihre Entwicklungen ebenfalls mit empirischen Erhebungen umfassend in den Blick zu nehmen. Erste Ergebnisse der NEPS-Studie sollen noch 2021 vorgelegt werden. Voraussetzung für diese und ggf. weitere Untersuchungen ist allerdings, dass die 
Länder den Forschungseinrichtungen einen schnellen und umfassenden Feldzugang gewähren, damit durch gute Teilnahmequoten auch belastbare Ergebnisse berichtet werden können.

Um die Schulen nicht mit vielen unkoordinierten Einzel-Untersuchungen zu überlasten, sollte die empirische und pädagogische Forschungskompetenz in Deutschland gebündelt und koordiniert eingesetzt werden. Bund, Länder und ggf. auch die im Bildungsbereich tätigen Stiftungen sollten diese Fragen vordringlich miteinander abstimmen und zügig zu Entscheidungen kommen.

\section{Wir brauchen dringend diagnosebasierte, wissenschaftlich fundierte Förderkonzepte und auf dieser Grundlage verbindliche zusätzliche Fördermaßnahmen!}

Für eine verlässliche Beschreibung und Bewertung der Folgen der Pandemie im Bildungsbereich ist es unerlässlich, auf der Ebene von länderrepräsentativen Studien Bildungsmonitoring zu betreiben. Zugleich müssen aber auch schon die nächsten Wochen und Monate und das kommende Schuljahr genutzt werden, um entstandene Lücken mit klugen, diagnosebasierten Fördermaßnahmen zu verringern und die Schüler ${ }^{\star}$ innen und ihre Familien psychisch und sozial zu stabilisieren. Wer helfen und unterstützen will, muss wissen, wo das Problem liegt und wer von ihm betroffen ist.

Keine Diagnostik ohne Förderung! Keine Förderung ohne Diagnostik! Bund und Länder planen zurzeit Maßnahmen für zusätzliche Lernangebote, um die Folgen der pandemiebedingten Schulschließungen zu kompensieren. Das kann man nur begrüßen! Allerdings müssen diese Angebote die von Defiziten betroffenen Schülerinnen auch tatsächlich erreichen und ihnen eine passgenaue, verbindliche Förderung ermöglichen. Im Schreiben von Staatssekretär Rachel (BMBF) vom 23.02.2021 an den Bildungsausschuss des Deutschen Bundestages heißt es dazu:

„Es muss damit gerechnet werden, dass sich in der Pandemie die Lernzeit der Schülerinnen und Schüler verringert hat. Das wird sich vermutlich auch in Lernrückständen niederschlagen. Wie groß diese Defizite sein werden, ist derzeit nicht bekannt."

Um Umfang und Inhalt der tatsächlichen Defizite zu erfassen, müssen die aktuellen Lernstände systematisch erfasst werden. Zentraler Maßstab sollten dabei die in den Bildungsstandards beschriebenen sprachlichen und mathematischen Kompetenzen sein. Daher sollten in den ersten vier Wochen des neuen Schuljahres bundesweit vorhandene und ergänzend länderspezifische Instrumente eingesetzt werden, um für alle Schülerinnen eine verlässliche Einschätzung zu möglichen Förderbedarfen zu ge- 
winnen. Die Länder sollten sich darauf verständigen, gemeinsam nach dem Vorbild NRWs die zentralen Vergleichsarbeiten VERA 3 und VERA 8 verbindlich auf den Beginn des Schuljahres 2021/22 zu verlegen und auf diese Weise eine Lernausgangslage für die Klassen 4 und 9 zu gewinnen. Die Durchführung auszusetzen oder freiwillig anzubieten verhindert eine flächendeckende, systematische und belastbare Basis, der zwingend verbindliche Fördermaßnahmen folgen müssen. Hier wird ohne Not eine große Chance vertan. Die in einigen Ländern bereits gewährte Unterstützung der Lehrpersonen bei der Korrektur und Auswertung durch Externe sollte ausgeweitet werden, um die Lehrpersonen zu entlasten und Kapazitäten für einen wirksamen Umgang mit den Ergebnissen zu schaffen.

Monitoring-Studien geben noch keine Hinweise auf individuelle Förderbedarfe, dazu braucht es andere Instrumente und Verfahren einer aussagekräftigen Individualdiagnostik. Zusätzlich braucht es eine konzertierte Aktion aller Länder, die zahlreichen vorhandenen, qualitätsgesicherten Diagnoseinstrumente zur klassenbezogenen oder individuellen Lernstandsdiagnostik für andere Jahrgangsstufen und Fächer auf einer digitalen Plattform für alle Schulen in Deutschland verfügbar zu machen. Die KMK sollte diese Verfahren und Instrumente unter Einbeziehung wissenschaftlicher Einrichtungen (z.B. dem DIPF) mit fundierten Empfehlungen für einen sinnvollen Einsatz in der Schule versehen und schnellstmöglich auf einer bundesweiten Plattform wie „Wir lernen online“ (WLO) oder „Mundo“ bereitstellen, damit sie schnell und unbürokratisch genutzt werden können.

Entscheidend wird sein, im Anschluss an die Diagnose wissenschaftlich fundierte Förderkonzepte und -maßnahmen umzusetzen. Zusätzliche Angebote, im Rahmen des Ganztags, aber auch an Samstagen oder in den Ferien, müssen geeignet und nachweisbar wirksam sein, um festgestellte Lernlücken und Versäumnisse anzugehen. Dazu wurden in den letzten Jahren im Rahmen diverser Forschungsprogramme wirksame Förderansätze identifiziert und erfolgreich evaluiert (z. B. https://biss-sprachbil dung.de/angebote-fuer-die-praxis/tool-dokumentation).

Auch stellt sich die Frage der Verbindlichkeit: Identifizierte Förderbedarfe für Risikogruppen müssen von den Schüler*innen auch wahrgenommen werden. Dazu sollten in Lern- und Fördervereinbarungen zwischen Schule und Schüler*innen unter Einbeziehung der Sorgeberechtigten additive individuelle Fördermaßnahmen vereinbart werden (vgl. z. B. Hamburg, Verordnung über die besondere Förderung von Schülerinnen und Schülern gemäß $₫ 45$ des Hamburgischen Schulgesetzes - VO-BF).

Die öffentlichen Schulen allein können dies nicht auffangen, die Angebote privater Nachhilfeinstitutionen und freier Jugendhilfeträger verfügen über die notwendige Expertise, Infrastruktur und Ressourcen und sollten regional in entsprechende für die Nutzer*innen kostenlose Angebote einbezogen werden. Die BuT-Lernförderung bietet hier eine gute Ressource, die bedarfsgerecht ausgeweitet werden müsste. Bei Bedarf 
müssen Möglichkeiten gefunden werden, den Eltern, die BuT-Lernangebote für ihre Kinder nutzen wollen, aber nicht zum Kreis der Anspruchsberechtigten gehören, die Selbstzahlungskosten zu ersetzen.

Viele Bundesländer unterbreiten bereits heute zusätzliche kostenlose Bildungsangebote in den Ferien. Das ist sinnvoll und sollte in allen Ländern stattfinden. Dabei geht es aber um mehr als das Bearbeiten von inhaltlichen Defiziten. Ausgeweitete Lernzeiten müssen für das Lernen auf allen Ebenen genutzt werden. Für viele Schüler*innen ist der Erfolg bei der kognitiven Leistungssteigerung davon abhängig, dass ihr Selbstvertrauen, ihre Lernmotivation und ihre Lernkompetenzen gestärkt werden. Bei der Förderung der Basiskompetenzen sollte fachliches Lernen mit überfachlichen Zielen verbunden werden, die Stärkung der personalen und sozialen Kompetenzen muss im Mittelpunkt stehen, um wieder Freude am Lernen zu ermöglichen und Selbstvertrauen aufzubauen. Gerade in der Pandemie ist deutlich geworden, wie entscheidend Selbständigkeit und Verantwortungsübernahme für die eigenen Lernfortschritte sind. Sie können nicht bei allen Schüler ${ }^{\star}$ innen vorausgesetzt werden und entwickeln sich nicht mit einmaligen Ferienkursen. Dazu brauchen wir ein längerfristiges Konzept für eine veränderte Lernkultur, gerade auch im Regelunterricht.

\section{Stark aus der Krise - innovative Strategien und zusätzliche Mittel sind erforderlich!}

Die Zeit bis zum Beginn des kommenden Schuljahres ist knapp bemessen, sie muss gut genutzt werden, um über kurzfristige Maßnahmen hinauszukommen. Wir müssen vor allem die Kinder und Jugendlichen, die am stärksten von der Krise getroffen wurden, auffangen, unterstützen und ihnen wieder eine Perspektive geben. Wir wollen, dass es jedem Kind, jedem Jugendlichen, im kommenden Schuljahr gelingt, seine Bildungsbiografie erfolgreich fortzusetzen, sowohl bei den Lerninhalten als auch bei kognitiven und sozialen Entwicklungen.

Um die pandemiebedingten Defizite auszugleichen und die Bildungschancen der Benachteiligten nachhaltig zu verbessern, brauchen wir ein passgenaues und angemessen ausgestattetes Förderprogramm von Bund und Ländern. Das Recht auf Bildung erfordert einen bundesweit geltenden Anspruch auf Mittel aus dieser zusätzlichen Förderung. Die Verteilung dieser Mittel sollte nicht nach dem sog. Königsteiner Schlüssel (Steueraufkommen und Einwohnerzahl) erfolgen, sondern anhand von Sozialindizes, die sich an der Zahl der Bedürftigsten orientieren. Als Zeithorizont für zusätzliche stützende Maßnahmen muss man dabei von mindestens zwei Schuljahren ausgehen. Dies muss gut kommuniziert werden, um Eltern und Schülerinnen zu vermitteln, dass eine Schuljahreswiederholung nur in Ausnahmefällen sinnvoll ist. Bei der Konzeption und Begleitung des Förderprogramms sollte die Expertise der 
Ständigen Wissenschaftlichen Kommission der Kultusministerkonferenz genutzt werden.

Die jetzt zu entwickelnden Konzepte und Maßnahmen sollten auch künftig eine gute Basis dafür bilden, Bildungsbenachteiligungen früh zu erkennen und ihnen kompensatorisch entgegenzuwirken. Dazu gehören vor allem deutlich mehr - der Rechtsanspruch auf Ganztag in der Grundschule muss zügig beschlossen und umgesetzt werden! - und qualitativ gute Ganztagsangebote, die genügend Gelegenheiten zur Förderung von Lernerfolgen benachteiligter Kinder und Jugendlicher bieten, aber auch auf Dauer angelegte Tutor*innenprogramme, Buddykonzepte, Lernpatenmodelle usw.

Schließlich könnten die angestrebten Ziele durch eine aktive Einbeziehung des Sozialraumes über Bildungsbündnisse mit den dort vorhandenen Akteuren der Zivilgesellschaft und die Nutzung der vor allem in städtischen Räumen vorhandenen vielfältigen Lernorte (Jugendverkehrsschulen, Gartenarbeitsschulen, Museen, Bibliotheken, außerschulische Lernorte, Schullandheime u.a.) schneller und nachhaltig erreicht werden. So könnte in der Krise auch eine Chance liegen, unser Schulsystem sozial gerechter weiterzuentwickeln.

\section{Literatur und Internetquellen}

Behörde für Schule und Berufsbildung Hamburg. (2011). Verordnung über die besondere Förderung von Schülerinnen und Schülern gemäß $\$ 45$ des Hamburgischen Schulgesetzes (VO-BF). Zugriff am 07.04.2021. Verfügbar unter: https://www.hamburg.de/content blob/3111190/e0d456469d131b3481f988166e091769/data/vo-besond-foerderung-schu eler-\$45.pdf.

Fickermann, D., \& Edelstein, B. (Hrsg.). (2020). „Langsam vermisse ich die Schule .... "Schule während und nach der Corona-Pandemie (DDS - Die Deutsche Schule, 16. Beiheft). Münster: Waxmann. https://doi.org/10.31244/9783830992318

Fickermann, D., \& Edelstein, B. (Hrsg.). (2021). Schule während der Corona-Pandemie. Neue Ergebnisse und Überblick über ein dynamisches Forschungsfeld (DDS - Die Deutsche Schule, 17. Beiheft). Münster: Waxmann. https://doi.org/10.31244/9783830993315

Hans-Jürgen Kuhn, geb. 1953, von 1989 bis 1990 Staatssekretär für Schule, Berufsbildung und Sport in Berlin; danach Leiter verschiedener Referate im Ministerium für Bildung, Jugend und Sport des Landes Brandenburg, zuletzt des Referats Qualitätsentwicklung und -sicherung, Schulentwicklung und Schulforschung; Gründungsgeschäftsführer des Instituts für Schulqualität der Länder Berlin und Brandenburg; Mitglied der Fokusgruppe Bildungspolitik der Heinrich-Böll-Stiftung. E-Mail: Kuhn.Hans-Juergen@web.de

Korrespondenzadresse: Gleditschstraße 37, 10781 Berlin 
| Hans-Jürgen Kuhn \& Michael Voges

Michael Voges, Dr., geb. 1952, von 2006 bis 2008 Staatsrat der Behörde für Bildung und Sport in Hamburg, danach Staatsrat in der Behörde für Soziales, Familie, Gesundheit und Verbraucherschutz; von 2010 bis 2011 Staatsrat der Finanzbehörde und von 2011 bis zu seiner Pensionierung Ende 2016 wieder Staatsrat der Behörde für Schule und Berufsbildung; Mitglied der Fokusgruppe Bildungspolitik der Heinrich-Böll-Stiftung.

E-Mail: mvoges@kabelmail.de

Korrespondenzadresse: Clausewitzstraße 8, 10629 Berlin 
Detlef Fickermann

\title{
Daten für Taten
}

\section{Verbesserung der Datengrundlagen für zielgerichteteres politisches Handeln zur Eindämmung und Bewältigung der Folgen der Corona-Pandemie}

\section{Zusammenfassung}

Daten bilden während der Corona-Pandemie die Basis für weitreichende politische Entscheidungen im Bund, in den Ländern und in den Kommunen und beeinflussen damit massiv den Alltag und die Gesundheit von Millionen Menschen. Jedoch fehlen viele Daten zum Infektionsgeschehen und vorhandene Daten aus anderen Bereichen werden nicht ergänzend für vertiefte Analysen genutzt. Beschrieben werden die aktuell vorhandenen Schuldaten sowie die darüber hinausgehenden Datenerfordernisse für das geplante Bund-Länder-Programm zur Schließung von coronabedingten Lern- bzw. Kompetenzlücken der Schüler*innen und dessen Evaluation. Ergänzend werden Ansätze zur Identifikation von kleinräumigen Infektionsclustern und zu berufsgruppen- und branchenspezifischen Auswertungen vorgeschlagen. Den Vorschlägen gemein ist die Nutzung von kleinräumigen Daten sowie die Inanspruchnahme eines vertrauenswürdigen Dritten, um datenschutzkonform (Individual-)Daten aus unterschiedlichen Quellen miteinander verknüpfen und für vertiefte Auswertungen als Grundlage für evidenzbasierte politische Entscheidungen zur Verfügung stellen zu können.

Schlüsselwörter: Corona-Pandemie, Lernlücken, Kompetenzstände, Bund-Länder-Programm, Infektionscluster, Vertrauenswürdiger Dritter

\section{Data to Act Upon}

Improving the Data Basis for Targeted Political Action to Contain and Overcome the Consequences of the Corona Pandemic

\begin{abstract}
This paper discusses the fact that in Germany, many of the extensive political decisions pertaining to the Corona Pandemic lack adequate evidence as a basis. Yet these decisions exert a massive influence on the daily life and health of millions of people. Data on the development of infections are, however, often incomplete, and chances to connect them to existing data from other areas are being missed. The article thus describes cur-
\end{abstract}


rently existing school data and the data requirements beyond them with regard to upcoming political decisions on the closing of students' competency gaps and its evaluation. Furthermore, approaches to identifying small-range infection clusters and possibilities for job-specific and sectoral analyses are proposed. The different suggestions share the ideas of using small-range data and utilizing a trusted third party to connect data from different sources and, in accordance with data privacy laws, make them available for deepened analyses, thus providing a basis for evidence-based political decisions.

Keywords: Corona Pandemic, learning gaps, competency levels, federal-state program, infection cluster, trusted third party

\section{Einleitung}

Daten, wie R-Wert, Sieben-Tage-Inzidenz, Belegungsquoten von Intensivbetten, Übersterblichkeit und einige andere bilden die Basis für weitreichende politische Entscheidungen im Bund, in den Ländern und in den Kommunen. Sie prägen in der Corona-Pandemie den Alltag und die Gesundheit von Millionen Menschen massiv. Jedoch fehlen viele Daten für eine vertiefte Analyse des Infektionsgeschehens oder sie werden nur unzureichend und kaum miteinander vergleichbar erhoben.

Der zweite Abschnitt des vorliegenden Diskussionsbeitrages ist daher den (Daten-) Grundlagen für das derzeit politisch diskutierte Bund-Länder-Programm zur Schließung von pandemiebedingten Lern- bzw. Kompetenzlücken gewidmet. Bei der Schaffung einer ausreichenden Datengrundlage für das geplante Programm und für dessen Evaluation ist der Einsatz eines vertrauenswürdigen Dritten ${ }^{1}$ für eine Verknüpfung von neu erhobenen Individualdaten - zum Beispiel von Kompetenzständen der Schüler*innen - mit vorhandenen Schüler*innenindividualdaten und mit kleinräumigen Daten ${ }^{2}$ der Bundesagentur für Arbeit oder der Statistischen Ämter des Bundes und der Länder zentral.

1 Ein vertrauenswürdiger Dritter oder eine vertrauenswürdige dritte Partei (engl. Trusted Third Party) ist eine dritte Instanz, der zwei Parteien vertrauen. Durch den Einsatz einer dritten, vertrauenswürdige Instanz lassen sich viele Datenschutzfragen gut lösen.

- Die TMF - Technologie- und Methodenplattform für die vernetzte medizinische Forschung e.V. (siehe https://www.tmf-ev.de/Home.aspx) setzt sich beispielsweise als Dachorganisation für die medizinische Verbundforschung in Deutschland in ihren Publikationen für den Einsatz von vertrauenswürdigen Dritten ein.

- Die Vertrauensstelle nach $\$ 98$ a HmbSG der Hamburger Behörde für Schule und Berufsbildung übernimmt bei der Nutzung von verknüpften schulischen Individualdaten für Monitoring- oder Forschungszwecke die Rolle eines vertrauenswürdigen Dritten (zu $\$ 98 \mathrm{a} \mathrm{HmbSG}$ vgl. http://www.landesrecht-hamburg.de/jportal/portal/page/bshaprod. psml? nid=3s\&showdoccase $=1 \&$ doc.id=jlr-SchulGHAV32P98a\&st=null).

2 Als kleinräumige Daten werden im Folgenden Daten auf der Ebene von Statistischen Bezirken bezeichnet, die Städte bzw. Gemeinden, Stadt- bzw. Gemeindebezirke und Ortsteile bzw. Ortschaften unterteilen, um vorhandene Rauminformationen für statistische Aussagen und raumbezogene Planungen nutzen zu können. Sie werden in der Regel durch sogenannte Baublöcke und Blockseiten weiter unterteilt. 
Im dritten Abschnitt wird ergänzend und exemplarisch an drei Beispielen gezeigt, wie von den Gesundheitsämtern erhobene Daten zu den Infizierten ebenfalls mit anderen Datenquellen verknüpft werden könnten, um zu einer vertieften Datengrundlage für zielgerichtete politische Entscheidungen kommen zu können.

Gemeinsam ist den Vorschlägen zum einen die Einschaltung eines vertrauenswürdigen Dritten bei der Verknüpfung von Daten, um den dabei zwingend notwendigen Datenschutz sicherzustellen, und zum anderen die Nutzung von kleinräumigen Daten der Statistischen Ämter des Bundes und der Länder sowie der Bundesagentur für Arbeit. Das Diskussionspapier schließt deshalb mit einem doppelten Plädoyer: Notwendig ist es erstens, den Zugang zu vorhandenen kleinräumigen Daten zu ermöglichen, und zweitens, die notwendigen Strukturen zu schaffen, damit diese unter Wahrung des Datenschutzes beispielsweise für das geplante Förderprogramm und dessen Evaluation sowie für vertiefte Analysen des Infektionsgeschehens als Basis für zielgerichteteres politisches Handeln zur Eindämmung und Bewältigung der Folgen der Pandemie genutzt werden können.

\section{Datengrundlagen für ein Bund-Länder-Programm zum Ausgleich pandemiebedingter Lern- bzw. Kompetenzrückstände von Schüler ${ }^{\star}$ innen und für dessen Evaluation}

\subsection{Vorhandene schulische Datenbestände}

\subsubsection{Schulstatistische Daten}

Die Amtschefskonferenz (AK) der Kultusministerkonferenz (KMK) hat 2003 die Einführung einer einheitlichen Datengrundlage für die Schulstatistik und einen Kerndatensatz (KDS) beschlossen. Die Einführung von Individualdaten und des Kerndatensatzes sollte die bis dahin übliche Erfassung von sogenannten Summendatensätzen für Planungs- und Statistikzwecke ablösen und eine länderübergreifende und einheitliche Datengrundlage für den Schulbereich schaffen. Zu dem Kerndatensatz gibt es eine FAQ (KMK, 2011). Darin enthalten ist auch die Version 3.0 des KDS. Im Jahr 2018 ist der KDS ebenso wie die FAQ überarbeitet worden; eine Veröffentlichung steht allerdings noch aus. Zusätzlich gibt es für die schulstatistischen Erhebungen der Länder einen Definitionenkatalog. Die aktuelle Fassung stammt aus dem Jahr 2020 (vgl. KMK, 2020a). Die Kommission für Statistik hat mehrmals der AK über den Stand der Einführung des KDS berichtet (zuletzt Ende 2018, vgl. KMK, 2018). Dem Bericht der Kommission für Statistik zufolge haben noch nicht alle Länder ihre Schulstatistik dem Beschluss von 2003 entsprechend umgestellt und gegenüber dem Bericht von 2015 stagniert die Umsetzung. 


\section{Am 15.10.2020 hat die KMK}

- eine Ländervereinbarung über die gemeinsame Grundstruktur des Schulwesens und die gesamtstaatliche Verantwortung der Länder in zentralen bildungspolitischen Fragen,

- politische Vorhaben zu der Ländervereinbarung sowie

- eine Verwaltungsvereinbarung zur Einrichtung einer ständigen wissenschaftlichen Kommission der KMK

beschlossen (vgl. KMK, 2020b, 2020c, 2020d). Zu den politischen Vorhaben zählt u.a. auch eine gemeinsame Strategie zur Datennutzung. Danach sollen die Länder

- die Schulstatistik auf Individualdatenerhebungen und den Kerndatensatz umstellen,

- sich verpflichten, die Individualdatensätze um Pseudonyme zu ergänzen, um länderinterne und länderübergreifende Längsschnittanalysen zu ermöglichen,

- die Entwicklung eines gemeinsamen, einheitlich strukturierten Datenmodells auf Basis des aktuellen Kerndatensatzes sicherstellen, um eine einheitliche Datenhaltung und Auswertung in den Ländern und damit die Vergleichbarkeit von Datenlieferungen zu ermöglichen und

- die Vergleichbarkeit der Daten durch einheitliche Festlegungen zu den Erhebungsmerkmalen und ihrer Definitionen sicherstellen.

Die Länder streben ferner eine Angleichung der Erhebungs-, Auswertungs- und Prognoseverfahren an (KMK, 2020c, S. 6) $)^{3}$.

\subsubsection{Schulstatistische Informationen der KMK zur Covid-19-Pandemie}

Seit der 46. Kalenderwoche (KW) veröffentlicht die KMK mit Unterbrechung von der 51. KW 2020 bis zur 7. KW 2021 Daten zum Infektionsgeschehen in den Schulen (siehe https:/www.kmk.org/dokumentation-statistik/statistik/schulstatistik/schulstatisti sche-informationen-zur-covid-19-pandemie.html). Berichtet werden für jedes Bundesland die Anzahl der Schulen ohne und mit eingeschränktem Präsenzbetrieb, die Zahl der den Schulen bekannten infizierten und die Zahl der in Quarantäne befindlichen Schüler*innen sowie die Anzahl der infizierten und der in Quarantäne befindlichen Lehrkräfte. Ein Blick auf die Fußnoten in den wöchentlich veröffentlichten Tabellen zeigt, dass die berichteten Daten länderübergreifend kaum miteinander vergleichbar sind. Seit der 8. KW 2021 werden die berichteten Daten durch eine kurze

3 Zur Nutzung von schulstatistischen Individualdaten zur Rekonstruktion von Bildungsverläufen für die Weiterentwicklung des Sozial- und Bildungsmonitorings in Nordrhein-Westfalen siehe beispielsweise Böttcher \& Kühne, 2016. 
Beschreibung der jeweiligen landesspezifischen Regelungen zur Schulorganisation ergänzt.

\subsubsection{Einführung eines nationalen Bildungsregisters}

Im Zusammenhang mit der Vorbereitung einer registergestützten Volkszählung haben das Statistische Bundesamt und das Bundesministerium für Bildung und Forschung (BMBF) Überlegungen zu einem Bildungsregister angestellt. Das BMBF hat dazu eine Machbarkeitsstudie beim Statistischen Bundesamt in Auftrag geben (vgl. Destatis, 2018, 2019). Neben dem Aufbau eines Bildungsregisters für den geplanten registerbasierten Zensus schlagen das BMBF und das Statistische Bundesamt die Einrichtung eines zentralen Bildungsverlaufsregisters beim Statistischen Bundesamt vor.

In einer neu eingerichteten Arbeitsgruppe besprechen Bund und Länder Fragen der Einrichtung des Bildungs- und des Bildungsverlaufsregisters. Die Länder präferieren nach derzeitigem Diskussionsstand eine dezentrale Lösung unter Beibehaltung ihrer föderalen Zuständigkeit für die Schuldaten.

Durch die im Gesetzgebungsverfahren für das Registermodernisierungsgesetz (RegMoG) vorgenommenen Änderungen und die Begrenzung der Nutzung der Steuer-ID als zentrale Personen-ID (Bundestag, 2020; Bundesrat, 2021) ${ }^{4}$ sind die bisherigen Überlegungen des BMBF und des Statistischen Bundesamtes für ein Bildungsverlaufsregister mit einer zentralen Personen-ID zunächst einmal obsolet. Wie es weitergeht, ist derzeit noch unklar. Ein bei Prof. Schnell (Universität Duisburg/ Essen) zu den Möglichkeiten einer Datenverknüpfung in Auftrag gegebenes Gutachten liegt bislang noch nicht vor.

Die bisherigen Überlegungen des BMBF und des Statistischen Bundesamtes sehen eine begrenzte Anzahl von Merkmalen für das Bildungsregister vor, die kaum vertiefte Auswertungen zum Bildungsgeschehen zulassen. So fehlen beispielsweise Merkmale zum sonderpädagogischen Förderbedarf oder zum Besuch eines ganztägigen Schulangebotes. Ebenfalls nicht berücksichtigt sind Leistungsdaten wie z. B. Abschlussnoten oder die Ergebnisse von nationalen oder internationalen Kompetenzfeststellungsverfahren bzw. Leistungsvergleichsstudien.

4 Nach der Verabschiedung im Bundestag am 28.01.2021 hat der Bundesrat dem geänderten Gesetzentwurf am 05.03.2021 zugestimmt, am 06.04.2021 ist das Gesetz im Bundesgesetzblatt veröffentlicht worden (vgl. https://www.bgbl.de/xaver/bgbl/start.xav\#_bgbl_ \%2F\%2F*\%5B\%40attr_id\%3D\%27bgbl121s0591.pdf\%27\%5D_1617798294916). 


\subsubsection{Daten nationaler oder internationaler Leistungsvergleichsstudien}

Die Daten für nationale (IQB-Bildungstrend) oder internationale Leistungsvergleichsstudien (PISA, IGLU, TIMSS, ICILS) werden von der Hamburger Niederlassung der International Association for the Evaluation of Educational Achievement (IEA-Hamburg, ehemals DPC) als Dienstleister erhoben (siehe https://www. iea.nl/de/studies/germany). Bestandteil der Erhebungen ist jeweils eine Schüler ${ }^{\star}$ innendemographieliste, die von den Schulen ausgefüllt und an die IEA-Hamburg übermittelt werden muss. Eine automatisierte Übernahme von in schulischen Verwaltungsprogrammen gespeicherten Daten ist in der Regel nicht vorgesehen. ${ }^{5}$

In den Schuljahren 2019/20 und 2020/21 sind die Erhebungen für den IQB-Bildungstrend ausgesetzt worden. Auch die Vergleichsarbeiten (VerA) wurden in den meisten Ländern nicht durchgeführt. Aus diesem Grund liegen in Deutschland auch kaum Daten zu coronabedingten Lern- oder Kompetenzrückständen vor. Ausnahmen bilden Kohortenvergleiche in Hamburg (vgl. Depping, Lücken, Musekamp \& Thonke, 2021) und Baden-Württemberg (vgl. Schult, Mahler, Fauth \& Lindner, 2021). Die vorgenommenen Kohortenvergleiche zeigen im Vergleich $\mathrm{zu}$ vorliegenden internationalen Befunden (vgl. Engzell, Frey \& Verhagen, 2020; Kuhfeld, Tarasawa, Johnson, Ruzek \& Lewis, 2020; Maldonado \& De Witte, 2020) vergleichsweise moderate pandemiebedingte Leistungs- und Kompetenzrückstände und auch die soziale Abhängigkeit der Leistungs- bzw. Kompetenzrückstände ist geringer als in den internationalen Studien.

\subsection{Notwendige Daten für ein Bund-Länder-Programm und dessen Evaluation}

Von Andreas Schleicher, der u.a. bei der Organization for Economic Cooperation and Development (OECD) für die PISA-Studien verantwortlich zeichnet, stammt der Ausspruch: „Ohne Daten sind Sie nur jemand der eine Meinung hat“. Angesichts der unbefriedigenden Datenlage zu pandemiebedingten Leistungs- und Kompetenzrückständen in Deutschland ist den Forderungen von Kuhn und Voges (in diesem Heft) und z.B. von Anger und Plünnecke (2021a, 2021b), die Lernausgangslagen der Schüler*innen als Grundlage für individuelle Fördermaßnahmen

5 Für die Erhebungen zu den IQB-Bildungstrends 2016 (Viertklässler*innen) und 2018 (Neuntklässler*innen) wurden von der Vertrauensstelle der Behörde für Schule und Berufsbildung die Schüler*innendemographielisten mit vorhandenen amtlichen Daten vorausgefüllt. Dadurch wurden zum einen die Schulen entlastet. Zum anderen konnten auf diese Weise die Hamburger Daten des IQB-Bildungstrends mit denen der Hamburger Kompetenzmessungen (KERMIT) verknüpft und so die eingesetzten Instrumente wechselseitig validiert sowie die (soziale) Selektivität des Rücklaufs genauer kontrolliert werden. In Bremen sind auf ähnliche Art und Weise die IQB-Daten mit den Schüler*innenindividualdaten verknüpft worden. 
zu bestimmen, unbedingt zuzustimmen. Auch Bundesbildungsministerin Karliczek hat jüngst in einem Interview Lernstandserhebungen als Grundlage für das geplante Bund-Länder-Programm gefordert (vgl. https://www.tagesspiegel.de/wissen/ ministerin-ueber-corona-hilfen-fuer-schueler-karlizcek-will-milliardenschweresnachhilfeprogramm/27048212.html). Einem Bericht der Süddeutschen Zeitung vom 28.03.2021 zufolge sei allerdings nun über diese Frage ein Streit zwischen Bund und Ländern entbrannt, denn viele Kultusminister*innen würden die von Karliczek geforderte Reihenfolge für falsch halten. „Erst zentral in ganz Deutschland den Lernstand zu erheben, wäre ein bürokratischer Irrsinn“, so wird Hessens Kultusminister Lorz zitiert (vgl. https://www.sueddeutsche.de/politik/schulen-in-der-pandemie-lernstandserhebungen-an-schulen-hessens-kultusminister-warnt-vor-buerokratischem-irrsinn1.5249633).

Notwendig sind belastbare Befunde, anstatt nur auf Meinungen, d.h. auf Einschätzungen von Lehrkräften und Eltern und teilweise auf Selbsteinschätzungen von Schüler*innen in Online-Erhebungen zu setzen. ${ }^{6}$ Es besteht nämlich sonst die große Gefahr, dass Angaben zu möglichen Lernrückständen wegen des teilweise ausgefallenen Präsenzunterrichts letztlich nur auf Angaben zu nicht behandelten Inhalten in Lehrplänen oder Lehrbüchern basieren und nicht auf tatsächlich erworbenen Kompetenzen.

Da die Lern- bzw. Kompetenzentwicklung der Schülerinnen während der Schulschließungen sicherlich höchst unterschiedlich verlaufen sein dürfte, bedarf es für den Ausgleich möglicher Lernrückstände oder besser: unzureichender Kompetenzentwicklungen, individueller Fördermaßnahmen, möglichst auf der Basis von Lernund Fördervereinbarungen (vgl. auch hierzu den Beitrag von Kuhn und Voges in diesem Heft).

Helbig (2021a, 2021b) diskutiert Vor- und Nachteile unterschiedlicher Maßnahmen zur Schließung von möglichen coronabedingten Lern- und Kompetenzrückständen, wie Nachhilfe bzw. zusätzlicher Unterricht am Samstag oder in den Ferien, Reduzierung der verpflichtenden Lehrplaninhalte, freiwillige Wiederholungen des Schuljahres, eine Wiederholung des Schuljahres durch alle Schüler*innen und eine Verlängerung des laufenden Schuljahrs bis Weihnachten. Er kommt dabei zu dem Schluss, es gebe keine einfache Lösung.

Dabei basieren die Inanspruchnahme von Nachhilfe bzw. Samstagsunterricht auf Freiwilligkeit. Eine verpflichtende Teilnahme an Förderangeboten außerhalb der Schulzeit, d.h. über die normale Stundentafel hinaus, dürfte vermutlich rechtlich nicht umsetzbar sein. Werden jedoch den Schüler*innen nur allgemeine Förder-

6 Für einen Überblick zu der Frage der eingeschätzten Lernrückstände vgl. Helm, Huber \& Loisinger, 2021, S. $26 f f$. 
angebote unterbreitet, besteht die große Gefahr, dass diese möglicherweise nicht von denjenigen Schüler*innen in Anspruch genommen werden, die sie am Dringendsten zum Ausgleich von entstandenen Lernrückständen bzw. zur Schließung von Lücken in ihrer Kompetenzentwicklung benötigen (zur Inanspruchnahme und Wirkung von extracurricularen Förderangeboten vgl. Lohaus \& Wild, 2021).

Damit zusätzliche Förderangebote möglichst effizient und effektiv hinsichtlich ihres Fördereffektes sein können, bedarf es einer äußerst intensiven Zusammenarbeit zwischen den Lehrkräften der Schüler*innen und denjenigen, die die Förderangebote anbieten. Hierzu ist ein Referenzrahmen, wie z. B. der aktuelle Kompetenzstand der zu fördernden bzw. in ihrer Kompetenzentwicklung zu unterstützenden Schüler*innen, erforderlich. Vorliegende Befunde beispielsweise zur Nachhilfe oder zur Hausaufgabenbetreuung in Schulen mit einem Ganztagsangebot verweisen nämlich auf eine geringe Wirksamkeit, wenn eine solche Abstimmung unzureichend ist.

Um die Wirksamkeit von Fördermaßnahmen zu überprüfen und ggf. nachsteuern zu können, bedarf es zusätzlich formativer Assessments. Angesichts einer coronabedingt vermutlich nochmals vergrößerten Varianz in den Lern- bzw. Kompetenzständen der Schüler*innen einer Klasse bzw. einer Lerngruppe dürften Klassenarbeiten allein sicherlich nicht ausreichen, um den Lernerfolg bzw. den Kompetenzzuwachs im Unterricht und durch die zusätzlichen Förderangebote festzustellen.

Kuhn und Voges (in diesem Heft) schlagen eine Verteilung der Mittel aus einem möglichen Bund-Länder-Programm an Hand von Sozialindices der Schulen vor (vgl. zu einem Überblick über Sozialindices Groot-Wilken, Isaac \& Schräpler, 2016). Leider liegen nicht in allen Bundesländern solche Sozialindices vor und sie werden in den Ländern höchst unterschiedlich berechnet, so dass es dringend erforderlich ist, sie für alle Schulen einheitlich zu bilden. Hier würde sich anbieten, bundesweit verfügbare Daten sowie einen einheitlichen und damit vergleichbaren methodischen Ansatz zu verwenden. Als theoretischer Bezugsrahmen könnte das Modell der Soziotope ${ }^{7}$ dienen (vgl. hierzu Bargel, Kuthe \& Mundt, 1977, 1978; Kuthe, Bargel, Nagl \& Reinhardt, 1979; Fickermann, 1999).

7 Bei den bisherigen Untersuchungen mit Soziotopen wurden Volkszählungsdaten verwendet. Da diese aktuell nicht zur Verfügung stehen, bietet sich die Nutzung von Daten der statistischen Ämter des Bundes und der Länder sowie von Daten der Bundesagentur für Arbeit (BA) an. Neben gemeindebezogenen Auswertungen stellt die BA für ca. 250 Gemeinden bzw. Städte auch kleinräumige Auswertungen auf der Basis einer vertraglichen Vereinbarung zur Verfügung. Die Auswertungen umfassen

- die sozialversicherungspflichtig und geringfügig Beschäftigten,

- die Arbeitslosen,

- die Arbeitslosengeldempfänger nach dem SGB III und

- die Leistungsberechtigten und Bedarfsgemeinschaften nach SGB II,

zumeist unterteilt nach Geschlecht, Alter, Deutsche/Ausländer, (ggf. zuletzt) ausgeübtem Beruf und höchstem Berufsabschluss. 
Zusammenfassend kann somit festgehalten werden: Ein mögliches Bund-LänderProgramm ist zwingend auf vorhandene und zusätzlich zu erhebende Daten angewiesen, wenn die Mittel gerecht verteilt sowie effizient und effektiv eingesetzt werden sollen. Und diese Daten müssen nicht nur als Individualdaten vorliegen, sondern sie müssen zusätzlich unter Wahrung des Datenschutzes für Monitoring-, Evaluationsund Forschungszwecke verknüpft werden.

\subsection{Etablierung eines vertrauenswürdigen Dritten für die Verknüpfung von schüler*innenbezogenen Individualdaten aus unterschiedlichen Quellen}

Nach datenschutzrechtlichen Diskussionen über die Erhebung und Verarbeitung von Schüler*innenindividualdaten einigte sich die Hamburger Behörde für Schule und Berufsbildung mit dem Hamburgischen Datenschutzbeauftragten 2012 auf die Vergabe eines Gutachtens an Herrn Prof. Roßnagel. Das Gutachten (vgl. Roßnagel, 2013) bildete dann die Grundlage für die Einführung des $\$ 98$ a in das Schulgesetz und die Einrichtung der Vertrauensstelle im Jahr 2016 (vgl. HmbSG, 2016).

Die Möglichkeiten der Verknüpfung von Daten wurde intensiv bei dem Evaluationsvorhaben EiBiSch (Evaluation inklusiver Bildung in Schulen) genutzt. Durch die Verknüpfung von Daten der Schulstatistik, von regelhaft erhobenen Schulleistungsdaten, von zusätzlich erhobenen Schulleistungsdaten von Schüler*innen am unteren Leistungsende, von erhobenen Daten zu den überfachlichen Kompetenzen und $\mathrm{zu}$ den „emotional-sozialen Schulerfahrungen“, von Einschätzungen der Lehrkräfte, der Schulleitungen und der Eltern sowie verschiedener weiterer Kontextvariablen konnten so die Effekte unterschiedlicher individueller und Strukturebenen in Quer- und Längsschnitten bestimmt werden. Insbesondere konnten dabei die Schulklasse als das entscheidende System für die Wirkungen der pädagogischen Veränderungen identifiziert werden (vgl. Fickermann \& Doll, 2015; Schuck, Rauer \& Prinz, 2019).

Die Nutzung von amtlichen Schuldaten zur Befüllung der Schüler*innendemographielisten durch die Vertrauensstelle für die Erhebungen in Hamburg zum Bildungstrend 2016 und 2018 wurden oben schon erwähnt.

Auch beim jüngsten Studienberechtigtenpanel des Deutschen Zentrums für Hochschul- und Wissenschaftsforschung (DZHW) wurde die Möglichkeit einer Verknüpfung der Erhebungsdaten mit vorhandenen Individualdaten der Hamburger Schüler*innen genutzt, um die schulischen und die gewünschten und realisierten nachschulischen Bildungswege der Schüler*innen in Abhängigkeit von individuellen sowie schulischen und regionalen Kontextmerkmalen analysieren zu können. 
Die bisherigen Erfahrungen zeigen ein deutlich ausgeweitetes Analysepotential durch verknüpfte Individualdaten aus unterschiedlichen Quellen sowohl für Monitoring- als auch für Forschungs- bzw. Evaluationszwecke.

\section{Nutzung von vorhandenen Datenbeständen für vertiefte Analysen des Infektionsgeschehens}

\section{Regionale Analysen des Infektionsgeschehens}

In den vergangenen Wochen ist in der Presse vermehrt über Auswertungen verschiedener Gebietskörperschaften zur regionalen Verteilung des Infektionsgeschehens berichtet worden. Beispielhaft sollen hier Berlin, Bremen und Köln genannt werden. Ein Ergebnis der Auswertungen ist die Vermutung, dass von der ersten Welle des Infektionsgeschehens eher „reichere“ Stadtteile betroffen waren als „ärmere“. Zurückgeführt wird dies u.a. auch auf Infektionen durch Reiserückkehrer*innen aus dem Wintersport (vgl. hierzu z.B. Doblhammer, Reinke \& Kreft, 2020). Die zweite (und dritte) Welle trifft nun überproportional „ärmere“ Stadtteile. Als Grund hierfür werden u.a. der generell bestehende Zusammenhang von Einkommen (und Bildung) und Gesundheit, beengte Wohnverhältnisse, fehlende Möglichkeiten des Arbeitens im Homeoffice aufgrund der ausgeübten Berufstätigkeit und die Notwenigkeit, öffentliche Verkehrsmittel nutzen zu müssen, angeführt. ${ }^{8}$

Um solchen möglichen Zusammenhängen nachgehen zu können, bietet es sich an, vorhandene kleinräumige Daten der amtlichen Statistik mit den Daten der Infizierten zu verbinden. Hierzu notwendig wäre es, jeder bzw. jedem Infizierten die Nummer des Statistischen Bezirks zuzuordnen, in dem sie bzw. er wohnt und darüber ihre bzw. seine Daten mit den vorhandenen Daten der Statistischen Bezirke zu verknüpfen. Selbstverständlich würden keine Individualdaten ausgewertet, sondern mit Aggregatdaten auf der Ebene der Statistischen Bezirke Clusteranalysen durchgeführt, um Gebiete mit ähnlichem Infektionsgeschehen zu identifizieren.

Als Datenquellen stünden beispielsweise Daten der Bundesagentur für Arbeit (s.o.), Daten der (fortgeschriebenen) Gebäude- und Wohnungszählung 2011, Bevölkerungsdaten, Angaben zur Siedlungsdichte u. ä. zur Verfügung.

8 Vorliegende Untersuchungen zur räumlichen Verteilung des Infektionsgeschehens und zu sozialen Einflussfaktoren in Deutschland (vgl. Doblhammer, Reinke \& Kreft, 2020 mit Hinweisen auf nationale und internationale Befunde) verwenden wegen des leichteren Datenzugangs als unterste Aggregationsstufe meist die Ebene der Kreise bzw. kreisfreien Städte (vgl. z. B. Ehlert, 2020; Scarpone, Brinkmann, Große, Sonnenwald, Fuchs \& Walker, 2020; Steiger, Mußgnug, \& Kroll, 2020; sowie Wahrendorf, Rupprecht, Dortmann, Schneider \& Dragano, 2021). Die zum Teil erheblichen Disparitäten innerhalb von Kreisen bzw. kreisfreien Städten bleiben damit unberücksichtigt. 
Sofern die Gesundheitsämter nicht in der Lage sind, die von ihnen erfassten Daten zu den Infizierten - zumeist Nachname, Vorname, Geschlecht, Alter, Wohnanschrift über die Wohnanschrift dem jeweiligen Statistischen Bezirk zuzuordnen, könnte diese Aufgabe von einer bzw. einem vertrauenswürdigen Dritten übernommen werden. Sie bzw. er bekäme beispielsweise die Angaben zu den Infizierten - selbstverständlich ohne Nach- und Vornamen - übermittelt und würde diesen die Kennziffer des Statistischen Bezirkes mit Hilfe eines Straßenrasters der Gemeinde zuordnen. Über die Kennziffer könnten dann von ihr bzw. ihm oder von einer zentralen Stelle der Gemeinde bzw. der Stadt, des Kreises, des Landes oder des Bundes ausgewählte kleinräumige Daten zugespielt und anschließend von einer dafür geeigneten Einrichtung z.B. einer Forschungseinrichtung - ausgewertet werden.

Der Wert solcher Auswertungen zur Identifizierung regionaler Cluster dürfte durch die fehlende Berücksichtigung von z.B. Beamten, Selbstständigen oder Nichtberufstätigen nicht wesentlich beeinträchtigt sein. Hilfsweise könnte die Quote der sozialversicherungspflichtig Beschäftigten als zusätzliche Variable bei den Clusteranalysen mitberücksichtigt werden.

Eine genauere Kenntnis des kleinräumigen Infektionsgeschehens würde es beispielsweise auch ermöglichen, genauer der Frage nachzugehen, ob Schulen mit einem gröBeren Anteil infizierter Schüler ${ }^{\star}$ innen durch einen Einzugsbereich mit einem insgesamt stärkeren Infektionsgeschehen gekennzeichnet sind. Wenn dem so wäre, würde dies darauf hindeuten, dass nicht primär Schulen als Infektionstreiber anzusehen sind, sondern Infektionen eher von außen in die Schulen hineingetragen würden.

\section{Berufsgruppenspezifische Analysen des Infektionsgeschehens}

Über berufsgruppenspezifische Risiken einer Infektion ist wenig bekannt. Die Presse berichtet hin und wieder von Infektionsclustern in Großbetrieben der Fleischindustrie oder auch in Logistikzentren. Zudem wird die Infektionsgefahr von Lehrkräften oder Erzieher*innen immer wieder breit öffentlich diskutiert.

Wenn die Erfassung des ausgeübten Berufes der bzw. des Infizierten durch die Gesundheitsämter aus Datenschutz- oder sonstigen Gründen nicht möglich ist, könnte eine Verknüpfung der Basisdaten von Infizierten mit dem Datenbestand der Bundesagentur für Arbeit (BA) beispielsweise $\mathrm{zu}$ den sozialversicherungspflichtig Beschäftigten oder zu sonstigen von der BA erfassten Personengruppen eine Lösung sein. ${ }^{9}$ Aus Datenschutzgründen müsste auch hier ein vertrauenswürdiger Dritter eingeschaltet werden.

9 Aussagen zur Infektion verbeamteter Lehrkräfte ließen sich auf diese Weise nicht treffen. Zu prüfen wäre, ob hierfür ergänzend auch Daten der Landesbesoldungsämter genutzt werden 
In der Meldung der sozialversicherungspflichtig Beschäftigten der Arbeitgeber*innen, die die BA über die Krankenkassen erhält, ist u. a. auch der aktuell ausgeübte Beruf in codierter Form enthalten. Eine Verknüpfung mit den Daten der Infizierten könnte über personenbezogene Merkmale (Nachname, Vorname, Alter, aktueller Wohnort) erfolgen. Da Eingabefehler der Gesundheitsämter zu den Angaben der Infizierten oder fehlerhafte Angaben der Infizierten selbst nicht ausgeschlossen werden können, könnten für eine Verknüpfung geeignete Techniken (Algorithmen) eingesetzt werden (vgl. hierzu z.B. Christen, Ranbaduge \& Schnell, 2020; Klumpe, Schröder \& Zwick, 2020; Schnell, 2019, 2020). Nach der Verknüpfung durch eine vertrauenswürdige Dritte bzw. einen vertrauenswürdigen Dritten könnte der um die personenbezogenen Angaben bereinigte Datensatz von ihr bzw. ihm einer dafür geeigneten Einrichtung - z.B. einer Forschungseinrichtung - für berufsspezifische Auswertungen zur Verfügung gestellt werden.

\section{Branchenspezifische Analysen des Infektionsgeschehens}

Das Verfahren für branchenspezifische Auswertungen könnte im Grundsatz dem für berufsspezifische Auswertungen entsprechen, da in den Meldungen der Arbeitgeber*innen für ihre sozialversicherungspflichtig Beschäftigten jeweils auch die Betriebsnummer enthalten ist. Mit deren Hilfe lassen sich jeder Meldung die Beschäftigungsanschrift und der Wirtschaftszweig zuordnen und auf diese Weise werden dann auch Auswertungen nach z. B. Wirtschaftszweigen und Regionen möglich.

\section{$4 \quad$ Zusammenfassung}

Den skizierten Datenerfordernissen für das geplante Bund-Länder-Programm und den drei im dritten Abschnitt behandelten Beispielen ist die Nutzung von überwiegend vorhandenen (Individual-)Daten aus unterschiedlichen Quellen für vertiefte Auswertungen als Grundlage für zielgerichtete politische Entscheidungen und deren Evaluation gemein. Eine Nutzung von verknüpften (Individual-)Daten aus unterschiedlichen Quellen ist datenschutzrechtlich nur mit Hilfe von vertrauenswürdigen Dritten möglich. Die Hamburger Vertrauensstelle nach $\$ 98$ a HmbSG könnte hierfür eine Art Blaupause sein. ${ }^{10}$ Zusätzlich erforderlich sind jedoch auch politische Entscheidungen, vorhandene (Individual-)Daten, wie z. B. Daten der sozialversi-

könnten. Erzieher*innen sind überwiegend Angestellte und damit sozialversicherungspflichtig.

10 Nachdem der Autor dieses Diskussionsbeitrages seine Überlegungen zu einem dezentral organisierten Bildungsregister sowohl in dem Workshop des BMBF (vgl. BMBF, 2018 und Fickermann, 2018a) als auch in der Kommission für Statistik der KMK vorgestellt hat (Fickermann, 2018b) bereitet er zur Zeit hierzu ein ausführliches Diskussionspapier vor, das die aktuelle Diskussion über die Einführung eines nationalen Bildungsregisters aufgreift und das in Kürze erscheinen wird. 
cherungspflichtig Beschäftigten, für Verknüpfungen und dadurch möglich werdende vertiefte Auswertungen zum Infektionsgeschehen (Identifikation von kleinräumigen Infektionsclustern, berufsgruppen- und branchenspezifische Auswertungen) und für das geplante Bund-Länder-Programm zur Verfügung zu stellen. Die Kultusminister ${ }^{\star}$ innen sind zudem gefordert, Leistungs- und Kompetenztests als Grundlage für individuelle Förderungen und formative Assessments zur Überprüfung von Lernfortschritten und Kompetenzentwicklungen im Unterricht und durch zusätzliche Förderungen einzuführen.

\section{Literatur und Internetquellen}

Anger, C., \& Plünnecke, A. (2021a). Schulische Bildung in Zeiten der Corona-Krise. Bildungsdefizite schnell beheben. Kurzstudie zum INSM-Bildungsmonitor 2021. Köln: Institut der Deutschen Wirtschaft. Zugriff am 26.03.2021. Verfügbar unter: https:// www.iwkoeln.de/fileadmin/user_upload/Studien/Gutachten/PDF/2021/Kurzstudie_ INSM_Bildungsmonitor.pdf.

Anger, C., \& Plünnecke, A. (2021b). Bildung: Verschärfung der Ungleichheit durch Schulschließungen vermeiden. (IW-Kurzbericht 19/2021). Köln: Institut der Deutschen Wirtschaft. Zugriff am 26.03.2021. Verfügbar unter: https://www.iwkoeln.de/fileadmin/ user_upload/Studien/Kurzberichte/PDF/2021/IW-Kurzbericht_2021-Schulschlie\%C3 \%9Fungen-neu.pdf.

Bargel, T., Kuthe, M., \& Mundt, J. W. (1977). Zur Bestimmung sozialisationsrelevanter Areale (Soziotope) - Modelle, Verfahren und Probleme. In H. J. Hoffmann-Nowotny (Hrsg.), Politisches Klima und Planung (Soziale Indikatoren, Band V, S. 199-254). Frankfurt/New York: Campus.

Bargel, T., Kuthe, M., \& Mundt, J. W. (1978). Die Indizierung von Soziotopen als Grundlage der Messung sozialer Disparitäten. In H. J. Hoffmann-Nowotny (Hrsg.), Messung sozialer Disparitäten (Soziale Indikatoren, Band VI, S. 43-92). Frankfurt/New York: Campus.

Böttcher, W., \& Kühne, S. (2016). Schulstatistische Individualdaten zur Rekonstruktion von Bildungsverläufen. Perspektiven für die Weiterentwicklung des Sozial- und Bildungsmonitorings in Nordrhein-Westfalen. (FGW-Studie Vorbeugende Sozialpolitik 03). Düsseldorf: FGW. Zugriff am 26.03.2021. Verfügbar unter: https://www.fgw-nrw. de/fileadmin/user_upload/FGW-Studie-VSP-03-Boettcher-A1-komplett-Web.pdf.

Bundesministerium für Bildung und Forschung (BMBF). (2018). Dokumentation der Ergebnisse des Expertenworkshops Bildungsregister am 20. November 2018. Berlin: BMBF.

Bundesrat. (2021). Gesetzesbeschluss des Deutschen Bundestages - Gesetz zur Einführung und Verwendung einer Identifikationsnummer in der öffentlichen Verwaltung und zur Änderung weiterer Gesetze (Registermodernisierungsgesetz - RegMoG). Drucksache 121/21. Berlin: Bundesrat. Zugriff am 26.03.2021. Verfügbar unter: https://www.bundes rat.de/drs.html?id=121-21.

Christen, P., Ranbaduge, T., \& Schnell, R. (2020). Linking Sensitive Data. Methods and Techniques for Practical Privacy-preserving Information Sharing. Cham: Springer Nature.

Depping, D., Lücken, M., Musekamp, F., \& Thonke, F. (2021). Kompetenzstände Hamburger Schüler*innen vor und während der Corona-Pandemie. In D. Fickermann \& B. Edelstein (Hrsg). Schule während der Corona-Pandemie. Neue Ergebnisse und Überblick 
über ein dynamisches Forschungsfeld. (DDS - Die Deutsche Schule, 17. Beiheft, S. 5179). Münster: Waxmann. DOI: https://doi.org/10.31244/9783830993315.03

Destatis (Statistisches Bundesamt). (2018). Einrichtung eines Bildungsregisters in Deutschland - Vorstudie. Wiesbaden: Destatis.

Destatis (Statistisches Bundesamt). (2019). Machbarkeitsstudie zur Einrichtung eines Bildungsregisters in Deutschland. Wiesbaden: Destatis.

Deutscher Bundestag. (2020). Entwurf eines Gesetzes zur Einführung und Verwendung einer Identifikationsnummer in der öffentlichen Verwaltung und zur Änderung weiterer Gesetze (Registermodernisierungsgesetz - RegMoG). Drucksache 19/24226. Zugriff am 26.03.2021. Berlin: Bundestag. Zugriff am 26.03.2021. Verfügbar unter: http://dipbt. bundestag.de/dip21/btd/19/242/1924226.pdf.

Doblhammer, G., Reinke, C., \& Kreft, D. (2020). Social Disparities in the First Wave of COVID-19 Infections in Germany: A County-scale Explainable Machine Learning Approach. Preprint (22.12.2020). Zugriff am 02.04.2021. Verfügbar unter: https://doi.org/ $10.1101 / 2020.12 .22 .20248386$.

Ehlert, A. (2020). The Socio-economic Determinants of COVID-19: A Spatial Analysis of German County Level Data. Preprint (07.07.2020) Zugriff am 02.04.2021. Verfügbar unter: https://www.medrxiv.org/content/10.1101/2020.06.25.20140459v1.full.pdf.

Engzell, P., Frey, A., \& Verhagen, M. D. (2020). Learning Inequality During the Covid-19 Pandemic. Preprint. Zugriff am 26.03.2021. Verfügbar unter: https://doi.org/10.31235/ osf.io/ve4z7

Fickermann, D. (1999). Grundschulzeugnis und Schulformwahlverhalten. Untersuchungen zur Leistungsselektivität beim Übergang in die Sekundarstufe I. In H. Weishaupt (Hrsg.), Zum Übergang auf weiterführende Schulen. Statistische Analysen und Fallstudien (Erfurter Studien zur Entwicklung des Bildungswesens, Band 7) (S. 113155). Erfurt: Pädagogische Hochschule.

Fickermann, D. (2018a). Möglichkeiten der Nutzung schulstatistischer Daten in einem Bildungsregister. Präsentation im Rahmen eines Workshops des Bundesministeriums für Bildung und Forschung am 20.11.2018.

Fickermann, D. (2018b). Möglichkeiten der Nutzung schulstatistischer Daten in einem Bildungsregister. Präsentation im Rahmen der Sitzung der Kommission für Statistik der Kultusministerkonferenz am 13.12.2018.

Fickermann, D., \& Doll, J. (2015). Potential und Technik der Verknüpfung von Befragungsdaten mit schulstatistischen Individualdaten und Leistungsdaten im Projekt EIBISCH. DDS - Die Deutsche Schule, 107 (4), 365-374. Zugriff am 26.03.2021. Verfügbar unter: https://www.waxmann.com/artikelART101810.

Groot-Wilken, B., Isaac, K., \& Schräpler, J.-P. (Hrsg.). (2016). Sozialindices für Schulen. Hintergründe, Methoden und Anwendung. Münster: Waxmann.

Hamburgisches Schulgesetz (HmbSG). (2016). \$ 98a - Vertrauensstelle. Zugriff am 07.04.2021. Verfügbar unter: http://www.landesrecht-hamburg.de/jportal/portal/page/ bshaprod.psml?nid=3s\&showdoccase $=1 \& d o c . i d=j l r-S c h u l G H A V 32 P 98 a \& s t=$ null.

Helbig, M. (2021a). Corona-Schuljahre - und wie weiter? Eine Auseinandersetzung mit den aktuellen Debatten zur Schließung der Lernlücken infolge der Corona-Schuljahre 2019/20 und 2020/21. Discussion Paper P2021-002. Berlin: WZB. Zugriff am 26.03.2021. Verfügbar unter: https://bibliothek.wzb.eu/pdf/2021/p21-002.pdf.

Helbig, M. (2021b). Als hätte es Corona nicht gegeben. Bildungspolitische Reaktionen auf Schulschließungen und Distanzunterricht. WZBrief Bildung, 43, März 2021. Berlin: WZB. Zugriff am 26.03.2021. Verfügbar unter: https://www.wzb.eu/de/publikationen/ wzbrief-bildung.

Helm, C., Huber S., \& Loisinger, T. (2021). Was wissen wir über Lehr-Lern-Prozesse im Distanzunterricht während der Corona-Pandemie - Evidenz aus Deutschland, 
Österreich und der Schweiz. Zeitschrift für Erziehungswissenschaft, Online First (04.03.2021). Zugriff am 26.03.2021. Verfügbar unter: https://link.springer.com/article/ 10.1007/s11618-021-01000-Z.

Klumpe, B., Schröder, J., \& Zwick, M. (Hrsg.). (2020). Qualität bei zusammengeführten Daten. Befragungsdaten, administrative Daten, neue digitale Daten: miteinander besser? (Schriftenreihe der ASI - Arbeitsgemeinschaft Sozialwissenschaftlicher Institute). Wiesbaden: Springer VS.

KMK (Sekretariat der ständigen Konferenz der Kultusminister der Länder in der Bundesrepublik Deutschland). (2011). FAQ's - Frequently Asked Questions zum Kerndatensatz und zur Datengewinnungsstrategie. Berlin: KMK. Verfügbar unter: https:// www.kmk.org/fileadmin/Dateien/pdf/Statistik/FAQ_KDS.pdf.

KMK (Sekretariat der ständigen Konferenz der Kultusminister der Länder in der Bundesrepublik Deutschland). (2018). Bericht der Kommission für Statistik über den Stand der Umsetzung von Individualdatenerhebungen und des Kerndatensatzes in der Schulstatistik 2018. Berlin: KMK.

KMK (Sekretariat der ständigen Konferenz der Kultusminister der Länder in der Bundesrepublik Deutschland). (2020a). Definitionenkatalog zur Schulstatistik 2020. Berlin: KMK. Zugriff am 26.03.2021. Verfügbar unter: https://www.kmk.org/fileadmin/ Dateien/pdf/Statistik/Dokumentationen/Defkat2020_Anlagen.pdf.

KMK (Sekretariat der Ständigen Konferenz der Kultusminister der Länder in der Bundesrepublik Deutschland). (2020b). Ländervereinbarung über die gemeinsame Grundstruktur des Schulwesens und die gesamtstaatliche Verantwortung der Länder in zentralen bildungspolitischen Fragen (Beschluss der Kultusministerkonferenz vom 15.10.2020). Berlin: KMK. Zugriff am 26.03.2021. Verfügbar unter: https://www.kmk. org/fileadmin/Dateien/veroeffentlichungen_beschluesse/2020/2020_10_15-Laenderver einbarung.pdf

KMK (Sekretariat der Ständigen Konferenz der Kultusminister der Länder in der Bundesrepublik Deutschland). (2020c). Politische Vorhaben zur „Ländervereinbarung über die gemeinsame Grundstruktur des Schulwesens und die gesamtstaatliche Verantwortung der Länder in zentralen bildungspolitischen Fragen" vom 15.10.2020 (Beschluss der Kultusministerkonferenz vom 15.10.2020). Berlin: KMK. Zugriff am 26.03.2021. Verfügbar unter: https://www.kmk.org/fileadmin/Dateien/veroeffentlichungen_be schluesse/2020/2020_10_15-Politische-Vorhaben-LV.pdf

KMK (Sekretariat der Ständigen Konferenz der Kultusminister der Länder in der Bundesrepublik Deutschland). (2020d). Verwaltungsvereinbarung zwischen den Ländern in der Bundesrepublik Deutschland zur Einrichtung einer Ständigen wissenschaftlichen Kommission der Kultusministerkonferenz (Beschluss der Kultusministerkonferenz vom 15.10.2020). Berlin: KMK. Zugriff am 26.03.2021. Verfügbar unter: https://www.kmk. org/fileadmin/Dateien/veroeffentlichungen_beschluesse/2020/2020_10_15-Beirat-Ver waltungsvereinbarung-LV.pdf

Kuhfeld, M., Tarasawa, B., Johnson, A., Ruzek, E., \& Lewis, K. (2020). Learning during COVID-19: Initial Findings on Students' Reading and Math Achievement and Growth (NWEA-Research Brief). Zugriff am 26.03.2021. Verfügbar unter: https://www. nwea.org/content/uploads/2020/11/Collaborative-brief-Learning-during-COVID-19. NOV2020.pdf.

Kuthe, M., Bargel, T., Nagl, W., \& Reinhardt, K. (1979). Siedlungsstruktur und Schulstandorte. Sozial-räumliche Gliederung der Städte mit Gesamtschulen in Nordrhein-Westfalen. Paderborn u. a.: Schöningh.

Lohaus, A., \& Wild, E. (2021). Extracurriculare Förderangebote für benachteiligte Kinder und deren Eltern: Ein Angebot-Aneignungs-Modell zur Inanspruchnahme und Wirkung. Zeitschrift für Pädagogische Psychologie (2021), 35, 1-10. https://doi.org/10. 1024/1010-0652/a000268 
Maldonado, J. E., \& De Witte, K. (2020): The Effect of School Closures on Standardised Student Test Outcomes (Discussion Paper Series DPS20.17). Leuven: KU. Zugriff am 26.03.2021. Verfügbar unter: https://lirias.kuleuven.be/retrieve/588087.

Roßnagel, A. (2013). Datenschutzrechtliche Bewertung spezifischer Fragen der Schulstatistik in Hamburg. Rechtsgutachten im Auftrag der Behörde für Schule und Berufsbildung der Freien und Hansestadt Hamburg. Kassel: Universität Kassel.

Scarpone, C., Brinkmann, S. T., Große, T., Sonnenwald, D., Fuchs, M., Walker, B. B. (2020). A Multimethod Approach for County-scale Geospatial Analysis of Emerging Infectious Diseases: A Cross-sectional Case Study of COVID-19 Incidence in Germany. International Journal of Health Geographics 19, 32. https://doi.org/10.1186/s12942-02000225-1

Schnell, R. (2019). Eignung von Personenmerkmalen als Datengrundlage zur Verknüpfung von Registerinformationen im Integrierten Zensus. (Working Paper Series No. WPGRLC-2019-01). Nürnberg: German Record Linkage Center. Zugriff am 26.03.2021. Verfügbar unter: https://duepublico2.uni-due.de/receive/duepublico_mods_00049551

Schnell, R. (2020). Privacy-preserving Record Linkage in the Context of a National Statistics Institute. (Working Paper Series "Joined Up Data in Government: The Future of Data Linking Methods”). Zugriff am 26.03.2021. Verfügbar unter: https://www.gov.uk/ government/publications/joined-up-data-in-government-the-future-of-data-linkingmethods/privacy-preserving-record-linkage-in-the-context-of-a-national-statistics-ins titute.

Schuck, K.-D., Rauer, W., \& Prinz, D. (Hrsg.). (2018). EiBiSch - Evaluation inklusiver Bildung in Hamburgs Schulen. Quantitative und qualitative Ergebnisse (HANSE Hamburger Schriften zur Qualität im Bildungswesen, Band 17). Münster: Waxmann. Zugriff am 26.03.2021. Verfügbar unter: https://www.waxmann.com/index.php?eID= download\&buchnr=3922.

Schult, J., Mahler, N., Fauth, B., \& Lindner, M. A. (2021). Did Students Learn Less During the COVID-19 Pandemic? Reading and Mathematics Competencies Before and After the First Pandemic Wave. Preprint (11.03.2021). https://doi.org/10.31234/osf.io/pqtgf

Steiger, E., Mußgnug, T., \& Kroll, L. E. (2020). Causal Analysis of COVID-19 Observational Data in German Districts Reveals Effects of Mobility, Awareness, and Temperature. Preprint (23.07.2020). Zugriff am 02.04.2021. Verfügbar unter: https://doi.org/10.1101/ 2020.07.15.20154476

Wahrendorf, M., Rupprecht, C. J., Dortmann, O., Schneider, M., \& Dragano, N. (2021). Erhöhtes Risiko eines COVID-19-bedingten Krankenhausaufenthaltes für Arbeitslose: Eine Analyse von Krankenkassendaten von 1,28 Mio. Versicherten in Deutschland. Bundesgesundheitsblatt 64, 314-321. https://doi.org/10.1007/s00103-021-03280-6

Detlef Fickermann, geb. 1952, leitete bis zu seinem Eintritt in den (Un-)Ruhestand im Oktober 2018 die Stabsstelle „Forschungskooperation, Datengewinnungsstrategie“ im Hamburger Institut für Bildungsmonitoring und Qualitätsentwicklung (IfBQ) und die Vertrauensstelle gemäß $\$ 98$ a des Hamburgischen Schulgesetzes. Ferner vertrat er bis zu diesem Zeitpunkt Hamburg in der Kommission für Statistik der KMK. Als assoziiertes Mitglied im Wuppertaler Institut für bildungsökonomische Forschung widmet er sich seit Februar 2019 wieder verstärkt wissenschaftlichen Fragestellungen.

E-Mail: Detlef.Fickermann@arcor.de

Korrespondenzadresse: Hohler Weg 6, 59174 Kamen 


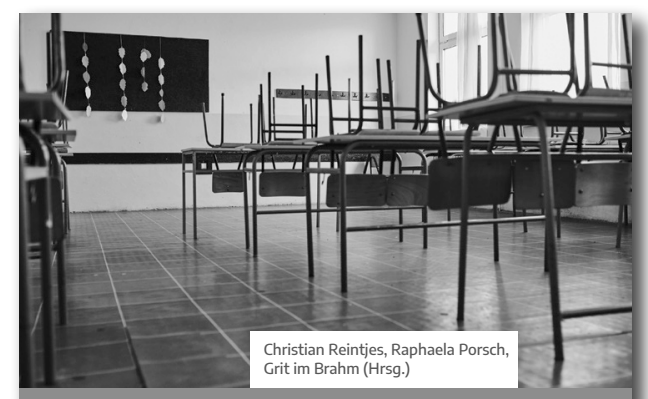

Das Bildungssystem in Zeiten der Krise

Empirische Befunde, Konsequenzen und Potenziale für das Lehren und Lernen

WAXMANN

\section{Mit Beiträgen von}

Gabriele Bellenberg, Grit im Brahm, Nina Bremm, Marike Bruns, Carina Caruso, Denise Demski, Benjamin Dreer, Matthias Forell, Patrick Gollub, Katja Görich, Klara Greffin, Edgar Hahn, Andreas Hülshoff, Livia JesacherRößler, Robin auf'm Kamp, Esther Dominique Klein, Nicola König, Bärbel Kracke, Dina Kuhlee, Ingrid Kunze, Markus Küpker, Philipp Matthes, Sonja Nonte, David Paulus, Raphaela Porsch, Torsten Porsch, Kathrin Racherbäumer, Christian Reintjes, Ricarda Rübben, Romy Schade, Jörg-Peter Schräpler und Marcel Veber
Christian Reintjes, Raphaela Porsch, Grit im Brahm (Hrsg.)

\section{Das Bildungssystem in Zeiten der Krise}

\author{
Empirische Befunde, \\ Konsequenzen und Potenziale \\ für das Lehren und Lernen
}

\author{
2021, 314 Seiten, br., 36,90 €, \\ ISBN 978-3-8309-4362-4 \\ E-Book: Open Access \\ https://doi.org/10.31244/9783830993629
}

Im Zuge der Covid-19-Pandemie standen Bildungsadministration, Schulleitungen, Lehrkräfte, Schülerinnen und Schüler und deren Eltern vor großen Herausforderungen. Es ging und geht aber nicht nur um die Frage der Organisation von Schule und Unterricht, der Sicherung von Abschlüssen oder der digitalen und technischen Ausstattung von (finanziell benachteiligten oder bildungsfernen) Familien, sondern auch um die Frage, wie der Gesundheitsschutz für Schülerinnen und Schüler sowie Lehrkräfte mit dem Recht auf Bildung und Teilhabe in Einklang zu bringen ist.

Ausgehend von einer (mehrebenenanalytischen) Bündelung aktueller empirischer Forschungsarbeiten zur Covid-19-Pandemie intendiert dieser Band, eine sachliche und evidenzbasierte Auseinandersetzung zur Reflexion der Covid-19-Pandemie zu initiieren sowie gegenwärtige Herausforderungen aus einer schulpädagogischen Perspektive zu diskutieren. 
vbw - Vereinigung der Bayerischen Wirtschaft e.V.

(Hrsg.)

\section{Führung, Leitung, Governance: Verantwortung im Bildungssystem}

2021, 280 Seiten, br., 22,90€,

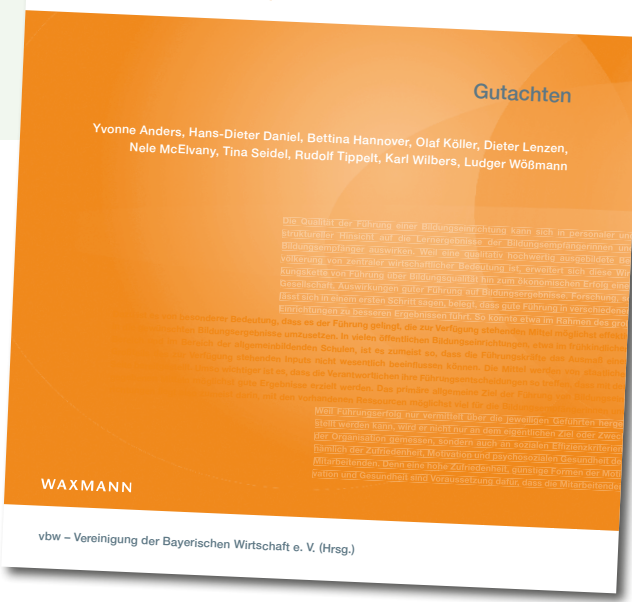

ISBN 978-3-8309-4400-3

E-Book: open access, doi.org/10.31244/9783830994008

Gute Führung führt zu besseren Bildungsergebnissen, die wiederum spürbare Auswirkungen auf das wirtschaftliche Wachstum und den Wohlstand einer Gesellschaft haben. Diese Zusammenhänge sind empirisch nachgewiesen, und dennoch sind Führungskräfte in deutschen Bildungseinrichtungen häufig nicht oder nicht ausreichend für diese wichtige Aufgabe qualifiziert. Zudem sind die Entscheidungsbefugnisse in vielen Bereichen, wie z. B. in der Personalauswahl und -honorierung, noch zu gering, um effektiv führen zu können.

Der Aktionsrat Bildung widmet sich der Frage, wie die Führung in deutschen Bildungseinrichtungen verbessert werden kann. Anhand der Wirkungskette - von der Leitung einer Bildungseinrichtung über das Lehrpersonal bis zu den Teilnehmerinnen und Teilnehmern der Bildungsangebote - geht er der Frage nach, welche Führungsstile den Zielen und Strukturen der einzelnen Bildungsphasen am besten entsprechen und welche Maßnahmen zu ergreifen sind, um Führung effektiv zu gestalten. Als zentrales Element benennt der Aktionsrat Bildung allgemeine sowie bildungsphasenspezifische Handlungsempfehlungen und richtet diese an die politischen Entscheidungsträger.

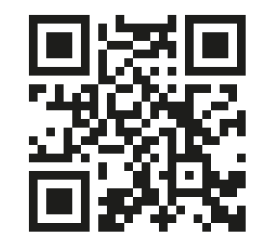

